\title{
The Impact of Dynamic Convergence on the Human Visual System in Head Mounted Displays
}

\author{
by \\ Ryan Sumner \\ A thesis \\ submitted to the Victoria University of Wellington \\ in partial fulfilment of the \\ requirements for the degree of \\ Master of Science \\ in Computer Graphics.
}

Victoria University of Wellington 2017 



\begin{abstract}
The Accommodation-Vergence Conflict (AVC) is a phenomenon in the area of Head-Mounted Displays (HMDs) and one of the key issues hindering the popularity of HMDs largely due to it causing a large number of users to suffer from simulator sickness. There have been several proposed solutions developed by previous researchers, including the introduction of 'Dynamic Convergence' (DC) which, addresses the AVC problem in terms of the vergence depth cue. DC also helps in the performance of binocular fusion when viewing at a close vergence depth. As of yet however, DC has not undergone detailed testing for a number of important cases, which limits the amount of data that has been collected on DC's interaction with the human visual system. In addition, no DC research as of yet has dealt with the effect of a change in vergence depth, and how that change in the vergence angle of the focal plane would effect a user.

Thus, this thesis adds to the growing body of research and knowledge in this field by implementing DC with the addition of some transitions between a change in vergence depth. This is done within the Unity3D game engine in order to further investigate the impact of DC with regard to viewing close virtual objects on HMDs through a number of cases. The added transitions are also tested to see if they have any beneficial effects for users when the vergence angle changes. The investigation is centered around a perception based performance/appreciation-oriented visual study whereby participants were asked about their ability to perform binocular fusion on close virtual objects that were either stationary
\end{abstract}


or moving and varying distances and speeds. Participants were also asked to report any symptoms of discomfort.

The research has adopted a mixed methodology experimental approach by conducting user experiments and surveys, before analysing the results through both in-depth quantitative statistical analysis and a variety of qualitative statistical techniques in order to measure and investigate the scale of the problem associated with the impact of DC on the human visual system in HMDs when viewing close virtual objects.

From the investigation it was confirmed that the approximate effective vergence depth range for DC was $0.3 \mathrm{~m}$ or less, with statistical significance confirmed at the $0.15 \mathrm{~m}$ distance. Participants reported having an easier time performing binocular fusion at these closer distances while DC was enabled. As a result of this, the majority of cases and scenarios did not report any significant negative responses in terms of discomfort symptoms. However attempts at improving DC with a transition between vergence depths were met with a mixed response from participants. While the need of a transition way be dependent on the user, there still exists some demand for one, thus it should still be available as an option. 


\section{Acknowledgments}

Firstly, I would like to thank my supervisor, Taehyun Rhee, for his guidance in directing me towards this interesting aspect of computer graphics for my thesis. And for his advice and feedback throughout the duration of the research.

Also, I would like to express my gratitude to all the participants who gave up their own time to take part in the user studies that were performed in this research. Without their provided information, none of the empirical research or findings would have been possible.

Similarly, I owe a great deal of gratitude to my father for providing advice and sharing his invaluable expertise in quantitative analysis. This allowed me to learn so much about the needed statistical techniques required to complete the defendable statistical analysis part of this thesis.

I would also like to thank my Victoria University student colleague, Kieran Carnegie, for sharing his insights and assistance, in particular for his help with my initial user test design.

Last but not least, I would like to thank my mother for her continual support and keeping me well looked after as always. 


\section{Contents}

$\begin{array}{llr}1 & \text { Introduction } & 17\end{array}$

1.1 Research Motivation . . . . . . . . . . . . . . 17

1.2 Research Objectives . . . . . . . . . . . . . . . . 19

1.3 Research Methodology . . . . . . . . . . . . . . . . 19

1.4 Contributions . . . . . . . . . . . . . . 20

2 Previous Research $\quad 23$

2.1 Head Mounted Displays . . . . . . . . . . . . . . . . 23

2.2 Simulator Sickness . . . . . . . . . . . . . . . . 24

2.2 .1 History . . . . . . . . . . . . . . . 24

2.2.2 General Causes . . . . . . . . . . . . 25

2.3 Accommodation-Vergence Conflict . . . . . . . . . . . . 27

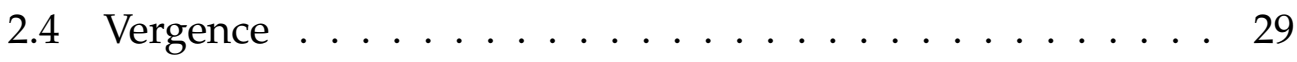

2.4.1 Hardware Solutions . . . . . . . . . . . . . 30 
2.4 .2 Software Solutions . . . . . . . . . . . 31

3 Dynamic Convergence Algorithm 35

3.1 Overview of the DC Algorithm . . . . . . . . . . 36

3.2 Gaze Estimation . . . . . . . . . . . . . . . . . 36

3.2.1 Finding the Vergence Depth . . . . . . . . . . . . 37

3.3 Virtual Camera Rotation . . . . . . . . . . . . . . . 39

3.4 Rotational Interpolation . . . . . . . . . . . . . . 42

4 Software Implementation $\quad 45$

4.1 Unity3D - Game Engine . . . . . . . . . . . . . . . 45

4.1 .1 Unity and Scripts . . . . . . . . . . . . 46

4.2 Overview ......................... 46

4.3 Implementing Gaze Approximation . . . . . . . . . . . . 47

4.4 Implementing Dynamic Convergence . . . . . . . . . . 48

4.5 Implementing Vergence Interpolation $\ldots . . . . . . . ~ 50$

5 A Quantitative Experiment on Dynamic Convergence 53

5.1 The User Test . . . . . . . . . . . . . . . . . . . . . 54

5.1 .1 Setup ........................ 54

5.1 .2 Procedure ..................... 56 
5.1 .3 Session One . . . . . . . . . . . . . . 57

5.1 .4 Session Two . . . . . . . . . . . . 60

5.2 Analysis ........................ 62

5.2.1 Statistical Methods . . . . . . . . . . . . . 63

5.2 .2 Invalid Results . . . . . . . . . . . . . . 68

5.2.3 Analysis of Session 1 Part 1 - Static Virtual Object . . 69

5.2.4 Analysis of Session 1 Part 2 - Instantaneous Movement Between Distances . . . . . . . . . . . . . . . 78

5.2.5 Analysis of Session 2 - Test Scenes with Interpolation 86

5.3 Discussion ............................. 95

6 Further Experimenting on Dynamic Convergence with Moving Objects $\quad 99$

6.1 The User Test . . . . . . . . . . . . . . . . . . . . . 100

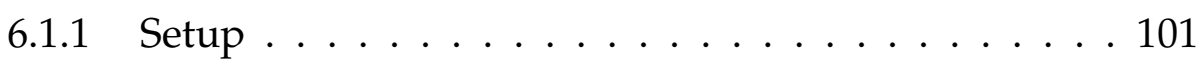

6.1 .2 Procedure .................... 101

6.1 .3 Transition Speed $\ldots . . \ldots 102$

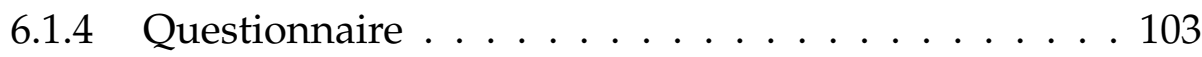

6.1 .5 Discrete Movement . . . . . . . . . . . . . . . . 104

6.1.6 Continuous Movement . . . . . . . . . . . 105 
6.2 Analysis $2 \ldots \ldots \ldots \ldots$. . . . . . . . . . . . . . . . . .

6.2 .1 Transition Speed . . . . . . . . . . . . . . . 106

6.2.2 Discrete Movement . . . . . . . . . . . . . . 107

6.2 .3 Continuous Movement . . . . . . . . . . . 116

6.2 .4 Summary ... . . . . . . . . . . . 119

6.3 Discussion . . . . . . . . . . . . . . . . . . 119

$\begin{array}{lll}7 & \text { Conclusion and Discussion } & 121\end{array}$

7.1 Summary . . . . . . . . . . . . . . . . . . 122

7.2 Limitations and Future Work . . . . . . . . . . . . . . 124

7.3 Conclusion . . . . . . . . . . . . . . . . . 126

A Code of the EGD-IDC System 135

B Advertisements, Forms and Questionnaires 143 


\section{Abbreviations}

3D Three Dimensional

AR Augmented Reality

AVC Accommodation-Vergence Conflict

DC Dynamic Convergence

FoV Field of View

IPD Inter-Pupillary Distance

IQR Inter-Quartile Range

SPSS Statistical Package for the Social Sciences

VE Virtual Environment

VR Virtual Reality 


\section{List of Figures}

2.1 A Diagram from Heilig Morton L's patent for a HMD in 1960 (left), a picture of the HTC Vive in 2016 (right) . . . . . 23

3.1 Several rays are cast in a straight line in the gaze direction to find the vergence depth of what is the current object of interest. . . . . . . . . . . . . . . 37

3.2 The curve of $\mathrm{A}=\arctan (\mathrm{IPD} / 2 \mathrm{dc})$ where IPD $=0.064$, the average IPD of a user. . . . . . . . . . . . . . . . . 39

3.3 The top image shows a representation of the human eyes converging on a virtual object though the virtual cameras while DC is disabled. The green arrows show the rotation of the eyes, while the red arrows show the rotation of the virtual cameras (none in this case). The bottom pair of images correspond to what would be seen by the respective eye in a HMD for a virtual scene (left image, left eye), the cube is at vergence depth of $0.15 \mathrm{~m}$ with no camera rotation (DC disabled). . . . . . . . . . . . . . . . . . . . . . . 40 
3.4 The top image shows a representation of the human eyes converging on a virtual object though the virtual cameras while DC is enabled. The green arrows show the angle the rotation of the eyes (none in this case), while the red arrows show the rotation of the virtual cameras. The bottom pair of images correspond to what would be seen by the respective eye in a HMD for a virtual scene (left image, left eye), the cube is at vergence depth of $0.15 \mathrm{~m}$ with some camera

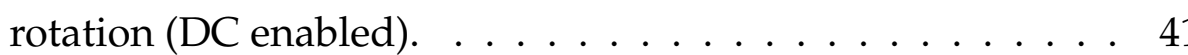

3.5 No Interpolation $\ldots \ldots \ldots$. . . . . . . . . . . . . . . 43

3.6 Linear Interpolation $\ldots \ldots 43$

3.7 Ease Interpolation . . . . . . . . . . . . . . . . . . . 44

5.1 Shows the study setup for a participant . . . . . . . . 56

5.2 The scene used in session one, which featured the same virtual object at a vergence depth of $0.15 \mathrm{~m}, 0.30 \mathrm{~m}, 0.45 \mathrm{~m}$ and $0.60 \mathrm{~m}$ respectively. . . . . . . . . . . 58

5.3 An excerpt of the questionnaire used in session one part one (see Figures B.4 and B.5 for the full questionnaire). The columns list the distances used. The rows are the symptoms participants were asked to rate on a likert scale of 1-5 in terms of severity. . . . . . . . . . . . . . 59

5.4 An excerpt of the questionnaire used in session one part two (see Figures B.4 and B.5 for the full questionnaire). The columns list the distance cases used. The rows are the symptoms participants were asked to rate on a likert scale of 1-5 in terms of severity. . . . . . . . . . . . . . . 59 
5.5 The scenes used in session two (in order) . . . . . . . . . . 60

5.6 An excerpt of the questionnaire used in session two (see Figures B.6 and B.7 for the full questionnaire). The columns list the distance scenarios used. The rows are the symptoms participants were asked to rate on a likert scale of 1-5 in terms of severity. . . . . . . . . . . . . . 61

5.7 Selection of the correct statistical tests $[8] \ldots \ldots 4$

5.8 Shows a section of the the relevant Chi-Square critical values that will used by the Friedman Test in this analysis. . . . 66

5.9 Shows the median discomfort scores across all test scenarios 70

5.10 Results of the Friedman test . . . . . . . . . . . 71

5.11 Results of the Wilcoxon Signed Rank Test . . . . . . . . . . . 72

5.12 Shows binocular fusion difficulty scores for all participants . 73

5.13 Shows box-plot of fusion difficulty scores with DC disabled. 74

5.14 Shows box-plot of fusion difficulty scores with DC enabled. 74

5.15 Results of the Friedman Test for DC ON / DC OFF . . . . . . 75

5.16 Results of the Wilcoxon Signed Rank Test for DC ON / DC OFF comparisons ............... 76

5.17 Shows the statistical significance results for the Wilcoxon Signed Rank Test for DC ON / DC OFF comparisons. As can be seen, there is significance at the distances of $0.15 \mathrm{~m}$ and $0.3 \mathrm{~m} \ldots \ldots \ldots \ldots \ldots 77$ 
5.18 Shows the Median symptom severity scores for the periodic and instantaneous movement of a close virtual object . . . 78

5.19 Friedman statistical test with DC disabled for instantaneous movement of close virtual objects indicated that there was a statistical significant difference in ease of fusion scores across the test scenes. . . . . . . . . . . . . . . 79

5.20 Shows median scores for instantaneous movement of close virtual objects test scene results with DC disabled. . . . . . . 80

5.21 Wilcoxon Signed Rank Test for the most significant instantaneous movement of close virtual objects between DC enabled and disabled. . . . . . . . . . . . . . . . . 81

5.22 Shows binocular fusion viewing difficulty scores with DC ON/OFF for all participants for instantaneous transitions. As can be seen values are higher at closer distances when DC is off.

5.23 Shows Friedman result when comparing DC ON/OFF for fusion difficulty only. Note the differences between the Mean Ranks between DC OFF/ON at the respective transition distances. This also shows there is a statistically significant difference in these sample population results. . . . . . . . 83

5.24 Wilcoxon Signed Rank Test for the most significant instantaneous movement of close virtual objects with DC disabled. 83

5.25 Shows the box-plot for binocular fusion viewing difficulty scores with DC disabled. . . . . . . . . . . . . . 85

5.26 Shows the box-plot for binocular fusion viewing difficulty scores with DC enabled. . . . . . . . . . . . . . 85 
5.27 Shows the median discomfort scores across all test scenarios for all types of interpolation . . . . . . . . . . . 87

5.28 Results of the Friedman test . . . . . . . . . . . . . . . 88

5.29 Results of the Friedman test for Eyestrain . . . . . . . . . . . 89

5.30 Show the box-plot for the severity of Eyestrain for No Interpolation. ..................... 90

5.31 Shows the box-plot for the severity of Eyestrain for Linear Interpolation. . . . . . . . . . . . . 90

5.32 Shows the box-plot for the severity of Eyestrain for Ease Interpolation. . . . . . . . . . . . . . . . 91

5.33 Shows the user preference percentage split for each mode of interpolation. . . . . . . . . . . . . . . 92

6.1 The distances used in the study, featuring the same virtual object at a vergence depth of $0.15 \mathrm{~m}, 0.30 \mathrm{~m}, 0.45 \mathrm{~m}$ and $0.60 \mathrm{~m}$ respectively. . . . . . . . . . . . . . . . 102

6.2 An excerpt for the discrete movement part of the questionnaire used in the second user evaluation (see Figure B.8 for the full questionnaire). The columns list the distance scenarios. The rows are the categories participants were asked to rank the three modes in terms of. . . . . . . . . . . . . . . 104

6.3 An excerpt for the continuous movement part of the questionnaire used in the second user evaluation (see Figure B.8 for the full questionnaire). The columns list the speed scenarios. The rows are the categories participants were asked to rank the two modes in terms of. . . . . . . . . . 106 
6.4 The percentage split of participants that preferred a particular linear interpolation speed. . . . . . . . . . . . 107

6.5 The number of participants that thought which mode(s) gave the least amount of general discomfort. . . . . . . . . . . . . 109

6.6 The number of participants that thought which mode(s) gave the most amount of general discomfort. . . . . . . . . . . 109

6.7 The number of participants that thought which mode(s) gave the least amount of eyestrain. . . . . . . . . . . . . 110

6.8 The number of participants that thought which mode(s) gave the most amount of eyestrain. . . . . . . . . . . . . . . 110

6.9 The number of participants that thought which mode(s) gave the least amount of nausea. . . . . . . . . . . . . . . . . . 111

6.10 The number of participants that thought which mode(s) gave the most amount of nausea.

6.11 The number of participants that thought which mode(s) gave the least amount of dizziness. . . . . . . . . . . . . . . . . 112

6.12 The number of participants that thought which mode(s) gave the most amount of dizziness. . . . . . . . . . . . . . . . 112

6.13 The number of participants that thought which mode(s) was the easiest to fuse with. . . . . . . . . . . . . . . . . . 113

6.14 The number of participants that thought which mode(s) was the hardest to fuse with. . . . . . . . . . . . . . 113

6.15 The number of participants that thought which mode(s) took the shortest amount of time to fuse with. . . . . . . . . . . . 114 
6.16 The number of participants that thought which mode(s) took the longest amount of time to fuse with. . . . . . . . . . . . 114

6.17 The number of participants that thought which mode(s) were overall, the best to use. . . . . . . . . . . . . . . . . 115

6.18 The number of participants that thought which mode(s) were overall, the worst to use. . . . . . . . . . . . . . . 115

6.19 The number of participants that thought which mode(s) gave the least amount of general discomfort. . . . . . . . . . . . . 117

6.20 The number of participants that thought which mode(s) gave the least amount of eyestrain. . . . . . . . . . . . . . . . 117

6.21 The number of participants that thought which mode(s) gave the least amount of nausea. . . . . . . . . . . . . . . . . . . . 117

6.22 The number of participants that thought which mode(s) gave the least amount of dizziness. . . . . . . . . . . . . . . . 118

6.23 The number of participants that thought which mode(s) was the easiest to fuse with. . . . . . . . . . . . . . . . . . 118

6.24 The number of participants that thought which mode(s) took the shortest amount of time to fuse with. . . . . . . . . . . . 118

6.25 The number of participants that thought which mode(s) were overall, the best to use. . . . . . . . . . . . . . . . . . . . 119

A.1 The Update function which executes every frame, the red box highlights what code was added to this function in the modified script OVRCameraRig.cs . . . . . . . . . . . 136 
A.2 Code for the Calculating the Vergence Distance based on the Estimated Gaze Assumption . . . . . . . . . . . . . . 137

A.3 Other math functions CalculateGazeDist() uses . . . . . . . 138

A.4 Code for handling the Raytracing in Unity3D . . . . . . . . 139

A.5 Code for handling starting and finishing angles of the virtual cameras . . . . . . . . . . . . . . . 139

A.6 The hierarchical setup used in the Unity3D Editor . . . . . 140

A.7 Used to update the position of the virtual eyes . . . . . . . 140

A.8 Left: the camera component of LeftCam. Right: the camera component of RightCam . . . . . . . . . . . . . . 141

A.9 Handles the interpolation of the virtual cameras . . . . . . 141

A.10 Used to the wanted position on the beizer curve . . . . . . 141

B.1 The advertisement used to recruit participants of the first

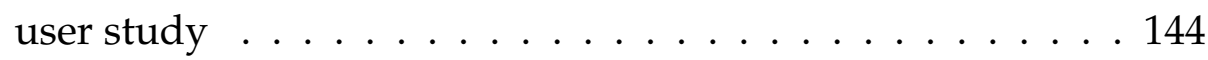

B.2 The advertisement used to recruit participants of the second user study . . . . . . . . . . . . . . . . 145

B.3 The consent form participants signed for both user studies . 146

B.4 Page 1 of 2. The Questionnaire used for session 1 part 1 of the first user study . . . . . . . . . . . . . 147

B.5 Page 2 of 2. The Questionnaire used for session 1 part 2 of the first user study . . . . . . . . . . . . . . . 148 
B.6 Page 1 of 2. The Questionnaire used for session 2 of the first user study . . . . . . . . . . . . . . . . . . 149

B.7 Page 2 of 2. The Questionnaire used for session 2 of the first user study . . . . . . . . . . . . . . . 150

B.8 The Questionnaire used for the second user study . . . . . . 151 


\section{List of Tables}




\section{List of Algorithms}

$1 \quad$ Calculating $d c \ldots \ldots \ldots \ldots \ldots \ldots \ldots \ldots$ 


\section{Chapter 1}

\section{Introduction}

\subsection{Research Motivation}

Head Mounted Displays (HMDs) have been gaining in popularity over recent years and one day they may prove become a viable alternative to the more conventional computer displays. Undoubtedly the new impetus in this area is being driven by heavyweight commercial investors such as Facebook, who acquired the virtual reality startup Oculus VR for 2 billion dollars in 2014. This in turn has led to a raft of competition from other large players such as Sony, Samsung and Google, all of whom are likely to increase research and development in order to produce higher quality and more affordable devices, which is great news for the consumer. Largely fueled by the ever-growing gaming industry, it is no surprise that the popularity and support of HMDs is because they are one of the most ideal systems for viewing VR applications (also known as Virtual Environments (VEs)). These Virtual Environments when coupled with input controls such as spatially tracked hand held controllers, offer a better interactive experience than most of the previous applications that used HMDs 
that tracked controller technologies separately.

A major practical issue with HMDs is that users commonly report symptoms of discomfort when using them. Such symptoms include headaches, nausea, dizziness and eye-strain [18]. These symptoms fall under the category of a condition called "simulator sickness" [31]. It has been discovered that simulator sickness is experienced by up to $80 \%$ of users of HMDs[42]. The specific subset of simulator sickness symptoms caused by visual stimuli is referred to as "visual discomfort" [10].

One of the more common and major causes of visual discomfort is the Accommodation-Vergence Conflict (AVC). AVC occurs in the human visual system when the accommodation depth cue and the vergence depth cue do not match. The vergence depth cue also ties in with our ability to perform binocular fusion which is important when wishing to view points of interest that are close to the viewer. With the previously mentioned tracked hand held controllers giving interaction within arms reach in virtual space, some interactions of future VEs might want to use virtual objects very close to the user. According to Percival's Zone of Comfort[40], a person is likely to suffer from visual discomfort in binocular vision within certain ranges, one of these ranges lies within the space very close to a person's eyes.

One of the more promising previously researched methods that helps with the vergence depth cue is called 'Dynamic Convergence' (DC). Dynamic Convergence is a software only solution that attempts to replicate the human eye vergence cue with the virtual cameras of a HMD. By having the virtual cameras doing the vergence cue, there is no longer a depth mismatch for the AVC and binocular fusion should be easier to perform on close virtual objects. However, from our knowledge DC has only been tested on a few cases with not much detail into its impact on discomfort symptoms; nor the distance range DC is most effective at. Further- 
more, no DC research as of yet has dealt with the effect of the change in vergence depth as the gaze point changes, and how that change in the rotation of the focal plane would effect a user. This research thus proposes to investigate the effective range DC is recommended for use, measure any impact it has on discomfort in more detail, identify any problems that the change in vergence depth has and try to mitigate them with some proposed transitions.

\subsection{Research Objectives}

The goal of this research is to investigate the use and effects of Dynamic Convergence for HMDs. This investigation will aim to determine:

- The distance range that DC aids the performance of binocular fusion when viewing virtual objects at close distances.

- In detail, if the use of DC has any positive or negative effects on viewing comfort while viewing close virtual objects.

- If there is a discomfort problem when a change in vergence depth happens and whether a transition is needed.

\subsection{Research Methodology}

In this research, the following steps are performed:

- Implemented DC with a number of transitions into the Unity3D game engine. 
- Designed and created virtual test scenes and questionnaire for testing.

- Recruited a number of participants to conduct the test and gather quantitative data.

- Analysed the results and deduced some defendable statistical inferences by the use of correct statistical techniques.

- Critiqued the methods used during the test to improve upon them.

- Redesigned a new questionnaire and test to expand the investigation.

- Recruited more participants to conduct the new test and gather qualitative data.

- Analysed the results using descriptive statistics.

- Conclude the overall findings of both tests.

\subsection{Contributions}

From the tests performed, this research has found:

- The discovery of the effective vergence depth range for Dynamic Convergence being at a distance of less than $0.30 \mathrm{~m}$ for aiding in binocular fusion.

- It was found that in the majority of cases Dynamic Convergence had no negative effects in terms of discomfort when viewing close stationary virtual objects. 
- Dynamic Convergence had a mostly positive response in terms of comfort when viewing close virtual objects that changed in vergence depth.

- A transition for an instant change in the vergence angle of Dynamic Convergence should be handled on a case by case basis, but having no transition seems to be more favourable. 


\section{Chapter 2}

\section{Previous Research}

\subsection{Head Mounted Displays}
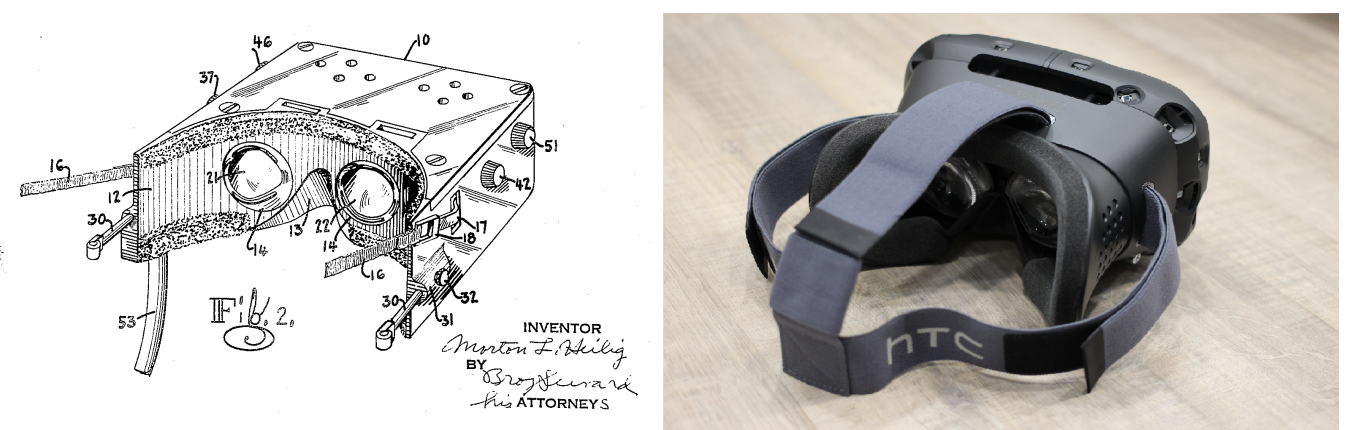

Figure 2.1: A Diagram from Heilig Morton L's patent for a HMD in 1960 (left), a picture of the HTC Vive in 2016 (right)

There was a patent made in 1960 which became the first formal description of a device that can be recognized as a HMD. The description of the patient describes a HMD as a device that is worn either by itself or as part of a helmet on the head of the user, such that it brings a display screen closely in front of each of the user's eyes [16]. Upon comparing 
the design of the original patient with the design of the current generation HMDs, the fundamental design is near identical even after even after a span of many decades in time (Figure 2.1).

No standardized system for categorizing devices used for viewing VEs exists yet, however many different systems have been proposed[9, 21]. In general there are two major classifications of HMD devices, Augmented reality (AR) and Virtual Reality (VR). An AR HMD takes an image of a real-world view with some modification and/or augmentation to that view, and a VR HMD occludes all view of the real-world and displays a completely virtual image.

For this research, the main focus will be on VR HMDs, as the majority of current research is aimed towards VR HMDs. In binocular HMDs, the images shown to each eye usually involve a small angular difference between them, this creates an impression of depth via stereopsis. Images rendered in this way are often referred to as "stereoscopic" content. By comparison, monoscopic content refers to images with no angular difference, for this the images displayed to each eye would be identical. The Oculus Rift DK2 that is used in this study is a VR binocular HMD, and was made to display stereoscopic content.

\subsection{Simulator Sickness}

\subsubsection{History}

In 1958, trainees using the helicopter training simulator called the "2-F2$\mathrm{H}$ hover trainer" [32] reported a series of discomforting reactions during their usage of the simulator. A vague definition of simulator sickness was used to describe these reported reactions: "the diverse signs or symptoms 
that have been experienced by flight crews during or after a training session in a flight simulator" [31].

The US army gives a more specific definition of simulator sickness: "discomfort [occurring] in a simulator of any kind" [22], no distinction was made based on the cause of the sickness symptoms, only that the symptoms happened in a "simulator". The term "visual discomfort" [10] is later introduced, which refers to simulator sickness which is caused purely by visual stimuli.

Symptoms of such discomfort can be split into three major categories [23]:

1. Oculomotor symptoms such as eye-strain, headaches and blurry vision.

2. Disorientation symptoms such as dizziness and vertigo.

3. Nausea symptoms such as changes in salivation and stomach awareness.

A single symptom can belong to multiple categories. For example, blurry vision is considered a symptom of both oculomotor and disorientation discomfort.

\subsubsection{General Causes}

Research into simulator sickness was done as early as 1958, the same year as the discomforting reactions from the "2-F2-H hover trainer" were reported. It was suggested at the time that the specific hardware components used in the 2-F2-H hover trainer such as low update frequency CRT 
monitors and helicopter motion bases were the causes of simulator sickness [32]. However many years later, identical symptoms were also reported with the usage of VR HMDs [19], this indicates that hardware components alone are not the sole factor in the cause of simulator sickness.

VR systems vary in terms of widely different designs, but they all have reports of simulator sickness, one factor these VR systems have in common is they all have a high level of immersion (or referred to as place illusion), which is defined as "the sensation of being in a real place"[41]. From having a high level of immersion, vection and expectation mismatch become some of the main causes of simulator sickness.

Vection is described as "the powerful illusion of self-motion" [17] and can occur when a large part a viewer's visual field moves. This may cause that viewer to believe that they are moving and the world around them is stationary [13]. So when a user is immersed in a virtual world, their location and orientation becomes defined by that virtual world they are in, as opposed to the location and orientation of their actual physical body [34]. So, when a large portion of a simulator's visual field moves, the user experiences an implied motion of their location and orientation, which creates this illusion of self-motion known as vection. In a study that induced vection, around $60 \%$ of participants reported motion sickness symptoms when subjected to a changing visual field while they remained stationary [44]. This suggests that there is a conflict between any perceived motions that a user has and the actual motions they have, which causes discomfort.

Similarly to how a conflict between perceived and actual motion can cause discomfort, motions that we do not expect can be sickening to. This is known as expectation mismatch. One example of this is when standing still on the deck of a ship, we normally expect a lack of motion due to standing still. However the ship can still rock in the water, caus- 
ing us to still experience motion, can cause seasickness. To further support this, a correlation was observed in regards to the use of the "2-F2-H hover trainer". The pilots who had more actual flight hours logged experienced higher severity of simulator sickness when using the simulator, when compared to pilots who had less flight hours logged [32]. From this correlation, a hypothesis was formed. The more experienced pilots had a better memory of the motions of a helicopter, so when they experienced the motions of the simulator, which conflicted with their expectations, they experienced Simulator Sickness. Following from the correlation, the less experienced pilots had not built up an expectation of such motions from a helicopter, thus they experienced less discomfort than the experienced pilots.

While it is know that vection and expectation mismatch also occur in HMDs [25], there is one major cause of user discomfort that is specific to HMDs. This is known as Accommodation-Vergence Conflict (AVC).

\subsection{Accommodation-Vergence Conflict}

The human visual system utilizes a number of difference cues to infer depth [35]. These cues can be split into two broad categories: Visual depth cues that involve inferring depth from visual stimuli and oculomotor depth cues that involve the physical responses of the ocular system in response to visual input. Oculomotor depth cues can be separated into three categories, accommodation, vergence and myosis. The depth cue of most interest in this research is vergence.

Due to the horizontal separation of the eyes, each eye needs to rotate inwards to form a single, clear image on the fovea (a small, central 
pit on the retina) of an object or point of interest (this can also be called the "fixation point" of both eyes). This rotation movement of the eyes is called vergence. Any misalignment of the fovea due to each eye focusing on a different point causes diplopia, or double vision. Objects behind or in front of the fixation point will form images on the retina that are not directly on the fovea, and are thus blurred. This provides a depth cue as a change in angular distance from the fovea affects the blur magnitude, which provides an indicator of the distance between the fixation point and the unfocused object.

Accommodation is another major oculomotor depth cue, it is the mechanism by which the eye slightly alters its shape to change its optical properties. This bring objects at varying distances into focus, so that they form a sharp image on the retina. Objects outside the current focal distance will be blurred, providing a cue as to their depth based on the degree to which they are blurred.

The accommodation and vergence responses are normally coupled. More specifically, any accommodative cue changes evoke vergence cue changes (accommodative vergence), and conversely any vergence cue changes evoke accommodative cue changes (vergence accommodation) $[14,30]$. For viewing in the real world, accommodation-vergence coupling is helpful because the focal and vergence distances are almost always the same no matter where the viewer looks.

However, In stereoscopic HMDs for VR, the accommodation and vergence depth cues do not match. The accommodation depth cue remains the same: the distance to the screen, however the vergence depth cue changes as the eyes converge to a wide variety of depths, depending on the virtual depths of the objects in the $\operatorname{VE}[37,39,33]$. This conflict of depth cues is results in the Accommodation-Vergence Conflict (AVC).

The AVC has effects beyond causing conflicting depth stimuli. 
During the mismatch binocular fusion times increases and fusion accuracy decreases, this decreases the perceived quality of immersive 3D experiences (discounting any visual discomfort)[5]. Long term adverse effects on the visual system are known to be a result of inconsistent accommodation and vergence cues [3], short term effects are also a concern, especially in tasks where accurate spatial reasoning is crucial. Because of these short term concerns, the US Air Force restricts pilots from flying real planes within 12-24 hours of exposure to a VR flight simulator [46].

Due to these adverse effects from the AVC, it is important to have correct or consistent accommodation and vergence cues for comfortable $3 \mathrm{D}$ viewing [35] and to avoid health risks on the human visual system.

\subsection{Vergence}

From multiple studies we know that the human eyes tend to focus and converge on objects or locations that are associated with the current task. For example, we tend to look at our hands or a tool as we use them, or we look at a location of where we are applying a tool (such as the location where you are writing with a pen) [7]. This task-oriented gaze of the human eyes also translates to VEs [2,36], as such some interactive tasks in VR may require the user to look at virtual objects that are very close to them. However there is a problem when trying to view close virtual objects in VR.

A HMD's left and right views represent what is seen by virtual cameras, which are co-located with the user's real eyes. For most VR HMD systems, both cameras are attached to a virtual head, which is controlled by a single motion sensor. The cameras are set to converge at some predefined distance, which is most commonly at infinity. Most HMDs also have 
the focal distance of their cameras fixed.

Because of such rigid settings of the display hardware, the pairs of images produced by the virtual cameras can have some significant differences from images that would be seen by real eyes. This is most apparent when looking at a virtual object of interest that is located farther or closer than the HMD's predefined vergence distance.

With the lack of flexible vergence in HMD-based VR systems, when objects of interest are at close range to the viewer, the use of parallel cameras can cause the following problems[38]:

- Double Vision: This is because the left and right object views appear on opposite sides of their respected image, such a disparity between the images makes the two images hard to fuse together.

- Stereo vision can easily be broken: If an object is centered in one image, the other easily goes off-screen.

- Diminishes Immersion: When a close object is on both images, it appears close to the black display borders, the edge of the HMD's lenses, which continuously reminds the user that their field of view is restricted.

- Affects all objects at close range: i.e. within hand's reach, including the hand itself, which is where the most accurate rendering and most precise object control are needed for task completion.

\subsubsection{Hardware Solutions}

Some previous research has been done on improving certain aspects of stereoscopic rendering on a hardware level. The results of which are de- 
signs and prototypes for HMD displays, which are capable of accommodating for very close objects by providing close to correct accommodation cues for multiple depths. Such examples include multifocal displays $[1,28]$ and an alternative lens system that makes use of active optical elements for producing images at various focus depths [27].

Dynamic Convergence (DC), adjusting vergence to the focal plane depending on what object of interest is being viewed, has been applied to an AR HMD prototype targeting medical applications [43]. The prototype used static video cameras, but dynamically adjusted the frame cropping to verge on the depth of the central object. Results from the user study indicate that DC does in fact mitigate AVC to some degree, but introduces a major problem referred to as the Disparity-Vergence Conflict. Adjusting the vergence to the focal plane means that, even though vergence no longer conflicts with accommodation, both cues now indicate the depth of the focal plane rather than the depth of the virtual object.

For optical-see-through HMDs for AR this conflict induces a mismatch between vergence for real-world and virtual objects. However, further studies with improved DC models will be required to determine whether the lack of a natural vergence cue will result in any depth misjudgments or stereo image fusion delays in opaque HMDs for VR [26]. However to support eye vergence on a physical level using hardware, a VR HMD needs to provide large nasal-side display areas, which is very difficult from an engineering standpoint. Thus, a software solution would be more preferable.

\subsubsection{Software Solutions}

Various DC models have been proposed that rely on algorithms that find the point or object of most interest in order to predict the gaze point of the 
user $[39,38,33,11]$. By predicting where the user is looking, the vergence of the the virtual cameras can be adjusted to the appropriate depth.

An experiment conducted by Sherstyuk et al. [38] used a DC system without eye-tracking to test user's hand-to-eye coordination. This was done by having users catch virtual butterflies with a virtual hand that was tracked to the user's own hand. The results of the experiment suggests that DC may improve performance in VR tasks on nearby objects.

Fisker et al. [15] were the first to use eye tracking integrated into a VR HMD to find the gaze point. They discovered that there was some quite severe eye strain with their initial DC model turned on. This prompted them to improve their DC system by filtering and smoothing out the vergence adjustments. They then tested their improved DC system inside a VE they created for a user study. However, their user study had conflicting results from the participants, thus no concrete conclusion was drawn from the study.

Later, Bernhard et al. [4] experimented with eye tracking and an auto-stereoscopic display with a similar DC model to Fisker et al. [15], and measured the fusion times of imagery with and without the DC enabled. They reported that there was an improvement in the fusion times with the DC enabled, but only for virtual objects placed in front of the focal plane. There were no significant improvements for virtual objects that were placed at or beyond the focal plane.

The studies investigating DC so far have shown there is at least some merit in a DC model enabled for viewing or interacting with close virtual objects or points of interest. However no research as of yet has dealt with the effect of the change in vergence depth as the gaze point changes, and how that change in the rotation of the focal plane would effect a user. This research thus proposes to investigate this effect, identify any problems that are found and test some transitions between the rotations in an 
aim to mitigate any potential discomfort. In addition, this research will also aim to investigate the effective range of DC use. Furthermore we will be measuring, in more detail than previous research, any discomfort symptoms that users may experience when viewing close virtual objects with and without DC. 


\section{Chapter 3}

\section{Dynamic Convergence Algorithm}

This research proposes to investigate the effects that DC has in terms of visual discomfort and the ease of performing binocular fusion when viewing close virtual objects. In order to accomplish this, a system that uses DC will be implemented using software only. Implementing and investigating a hardware-based system that assists with vergence was ruled out due to the difficulty of the acquisition of said hardware. In order to find the vergence depth, the DC system would also need to estimate a user's gaze without the use of external hardware such as an eye-tracker.

As part of the investigation into DC, the effects of changes to vergence depth while using DC is looked into. There has been little research done on these effects, as such users may experience some visual discomfort. For this, some proposed solutions will be implemented for testing. The purpose of these solutions are to aid those who are using DC to handle instant changes in vergence depth.

In this chapter, the algorithms that make the core of the system used are introduced and explained. The implementation of these algorithms will be later discussed in Chapter 4 . 


\subsection{Overview of the DC Algorithm}

The DC algorithm consists of three major parts:

- Gaze Estimator

- Virtual Camera Rotation

- Rotational Interpolation

The gaze estimator uses information from the VE and the HMD's orientation to estimate the user's gaze. Using this estimate, the vergence depth that the user should be focusing to is calculated and passed to the Virtual Camera Rotation algorithm. The Virtual Camera Rotation algorithm then rotates the focal planes of each of the HMD's lenses in their respected nasal direction. This rotation can then have a transition from one angle of vergence to the next in the form of an interpolation.

\subsection{Gaze Estimation}

In typical gaming conditions, approximately $86 \%$ of a user's fixation time, and $82 \%$ of their total viewing time is spent looking the centre of the screen $[29,24]$. From this observation, we can assume that the user is mainly focus looking at the centre of the screen without any other stimulus that would shift their focus (for example a cross-hair). With this assumption, it is possible to construct a DC algorithm that is gaze dependent without the use of eye tracking. Currently there are no consumer level HMDs that have been released that have inbuilt eye tracking, so the gaze assumption is the only feasible way to obtain the user's point of fixation for the DC algorithm without the need for any external hardware devices. This allows the the system to remain as a purely software solution. 


\subsubsection{Finding the Vergence Depth}

Since it is assumed that the user will always be looking at the centre of the screen, the ideal vergence depth $d c$ is the depth of the current object of fixation, which via the gaze assumption, is among the central pixels of the screen. To find $d c$ among the central pixels, a number of rays are cast from the head direction and orientation of the user in order to cover the central region of the user's gaze. 15 rays was considered to be a good compromise between having enough accurate coverage of the user's gaze and the amount of computational resources required. The exact position that the rays are cast from, are dependent on the user's Inter-Pupillary Distance (IPD), the rays are cast in the shape of a 3 ray tall 5 ray wide box at regular intervals (see Figure 3.1). The width of this ray box is the IPD of the user (which on average is $65 \mathrm{~mm}$ ), while the height of the box is $30 \mathrm{~mm}$, which approximately the height of the externally visible part of the human eye.
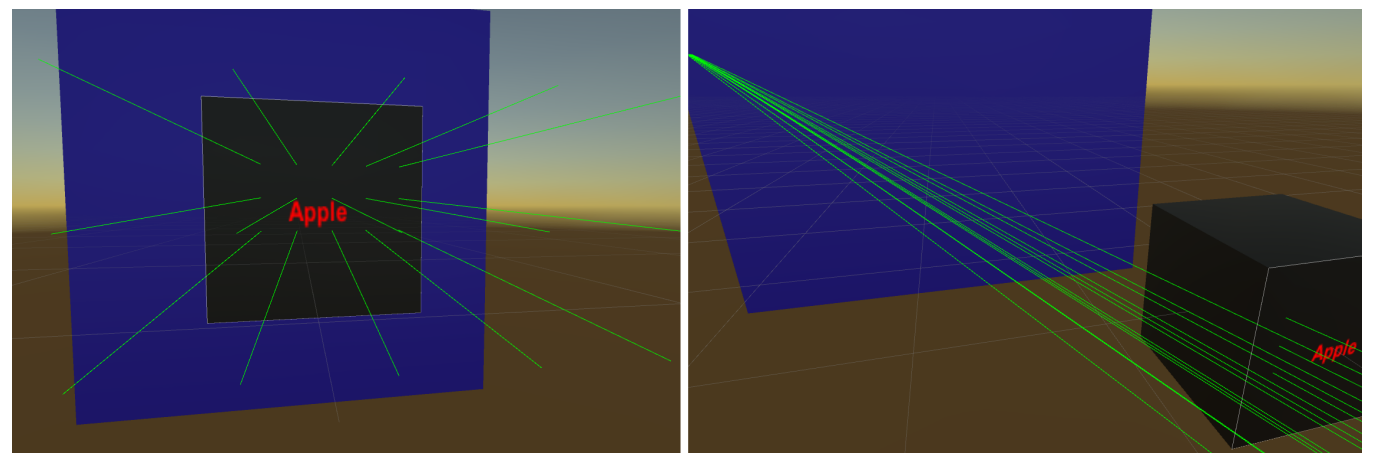

Figure 3.1: Several rays are cast in a straight line in the gaze direction to find the vergence depth of what is the current object of interest. 
Let $d$ be the set of fifteen ray intersection depths.

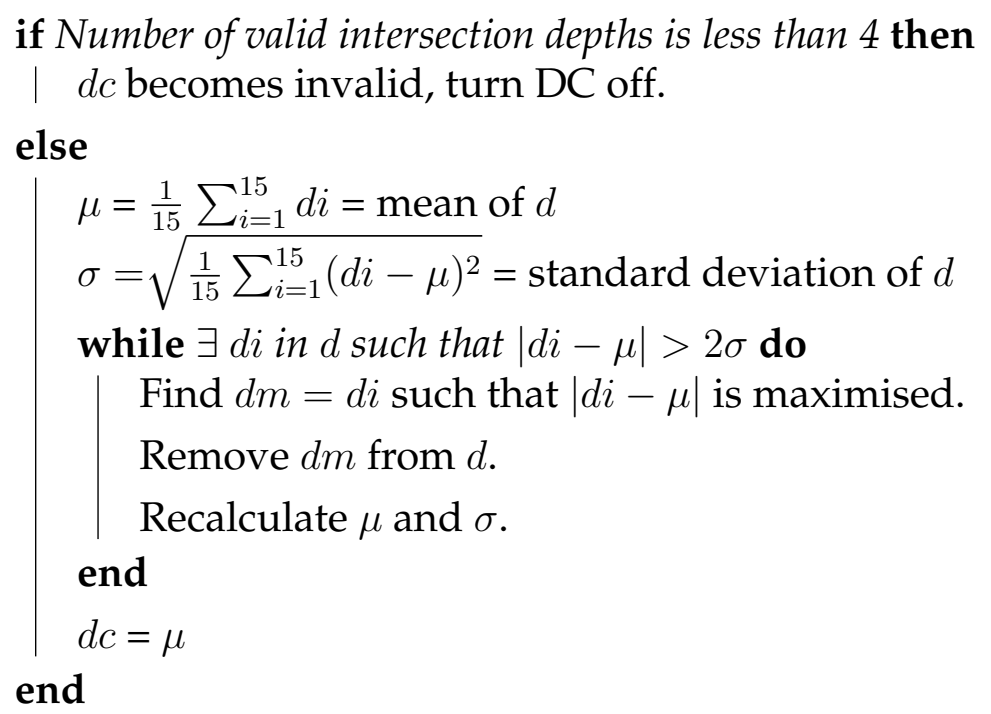

Algorithm 1: Calculating $d c$

Algorithm 1 shows how $d c$ is calculated. The theory is that the object of interest will take up more of the central region of the user's Field of View (FoV), thus more rays will intersect with the object of interest. The algorithm also removes depths that are outliers, such as rays that do not hit the intended object of interest. These rays would intersect at depths too close/far from the intended object of interest such that it would heavily impact the mean depth and thus give an unwanted $d c$. Thus it is important that outliers outside of 2 standard deviations from the mean are removed.

Preliminary tests deduced that there had to be a minimum number of valid ray intersections before DC should be turned on. A minimum threshold of 3 or less valid ray intersections would allow one column of rays to turn on $\mathrm{DC}$, which did not look or behave correctly in testing. Thus, it was deemed that a minimum threshold of 4 ray intersections was the correct number in producing the wanted behaviour. So if the minimum threshold of ray intersections is not meet for any virtual object, then $d c$ is marked as invalid and DC is disabled (the user would be looking at the sky-box of a VE for example). 


\subsection{Virtual Camera Rotation}

With $d c$ found we can calculate the vergence angle to the object of interest using Equation 3.1.

$$
A=\arctan (I P D / 2 d c)
$$

Where IPD is the Inter-Pupillary Distance that is calibrated to the user. Finally, the virtual cameras are rotated inwards respectively by the vergence angle $A$. The virtual cameras are facing the negative $Z$-direction and are separated by the IPD.

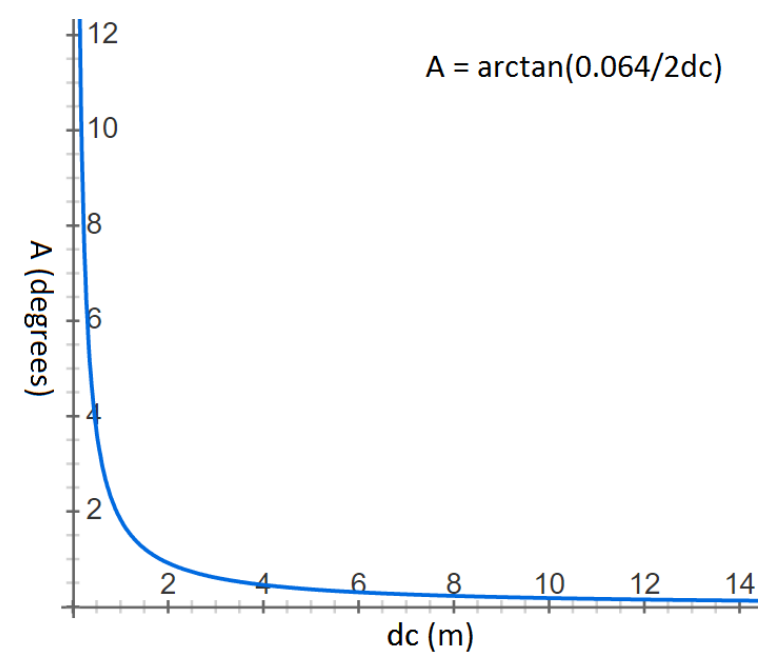

Figure 3.2: The curve of $A=\arctan (I P D / 2 d c)$ where IPD $=0.064$, the average IPD of a user.

Figure 3.2 shows the average vergence angle at the appropriate vergence distance. From this it can be noted that the vergence angle $A$ only begins to become significant (greater than 2 degrees) when $d c$ is less than 1 metre, which is the range of vergence depth at our investigation is most interested in. 


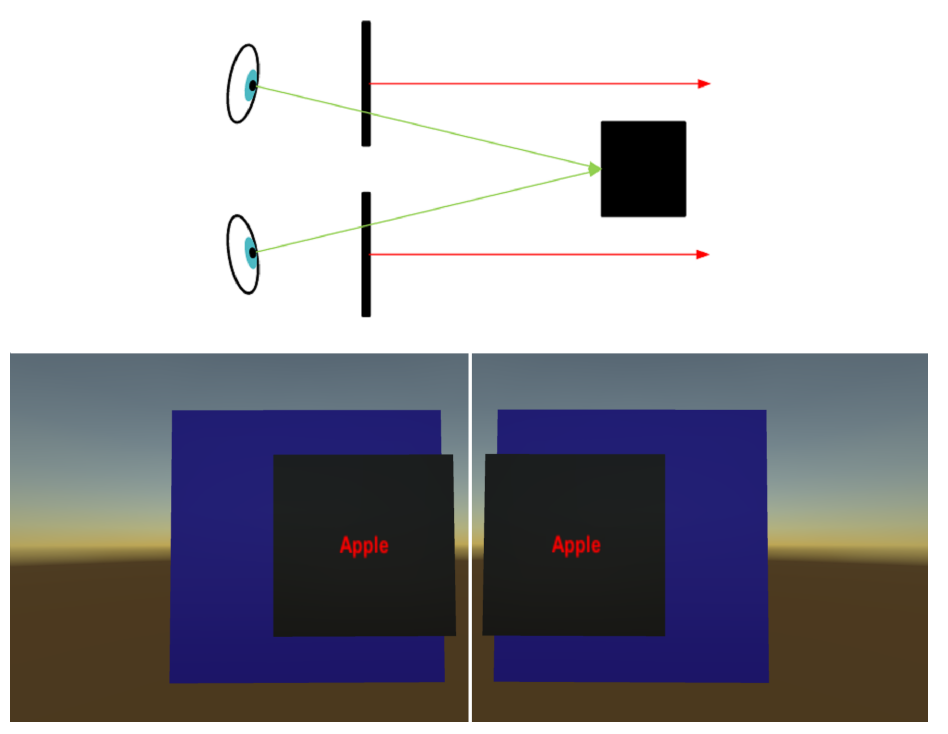

Figure 3.3: The top image shows a representation of the human eyes converging on a virtual object though the virtual cameras while DC is disabled. The green arrows show the rotation of the eyes, while the red arrows show the rotation of the virtual cameras (none in this case). The bottom pair of images correspond to what would be seen by the respective eye in a HMD for a virtual scene (left image, left eye), the cube is at vergence depth of $0.15 \mathrm{~m}$ with no camera rotation (DC disabled).

Figures 3.3 and 3.4 show the differences between having DC enabled and disabled in terms of virtual camera rotation and human eye rotation. If DC is turned off while a user is looking at a close virtual object, the user will have to perform convergence with their own eyes. However the act of performing convergence requires notable effort from the eyes at extremely close distances. In addition, the user's FoV is restricted by viewing through parallel cameras. This makes the black display borders, the edge of the HMD's lenses, visible which adds to the disparity between the images seen in the left and right eye. Both the extra effort when verging and the increased disparity between the stereo images, make it a lot more difficult for the user to perform binocular fusion. 

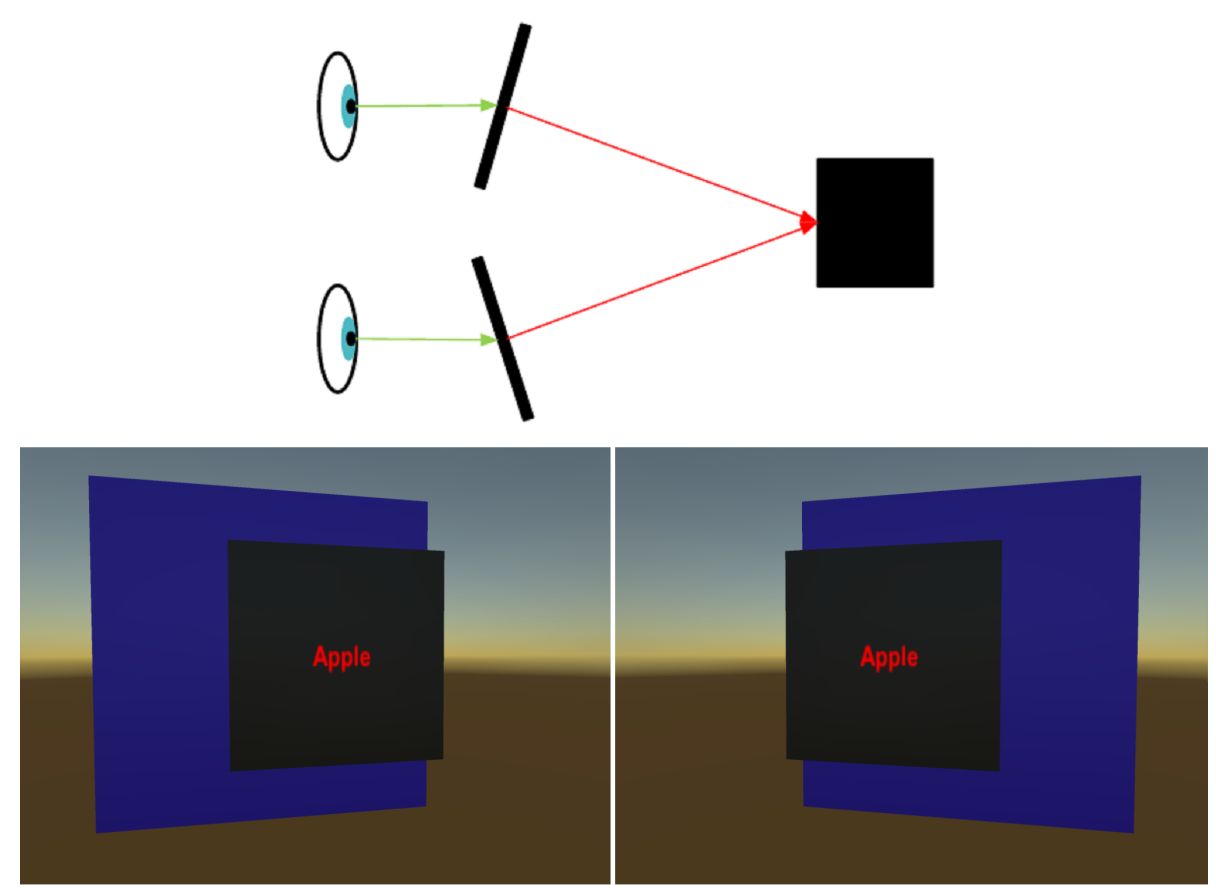

Figure 3.4: The top image shows a representation of the human eyes converging on a virtual object though the virtual cameras while DC is enabled. The green arrows show the angle the rotation of the eyes (none in this case), while the red arrows show the rotation of the virtual cameras. The bottom pair of images correspond to what would be seen by the respective eye in a HMD for a virtual scene (left image, left eye), the cube is at vergence depth of $0.15 \mathrm{~m}$ with some camera rotation (DC enabled).

By having DC enabled, the object of interest becomes centralized on each of the HMD's screens, this allows the user's FoV to become unobstructed by the black borders of the HMD's lenses. This, in addition to the user no longer needing to perform vergence with their eyes, allows the user to perform binocular fusion more easily. 


\subsection{Rotational Interpolation}

So far there has been little research done into the effects of a change to vergence depth while using DC. The most extreme case would be an instant and large change in vergence depth, resulting in an instant change in the vergence angle. From Figure 3.2 we can see that if a vergence depth is extremely close (less than $0.5 \mathrm{~m}$ for example), it wouldn't take much of a change in vergence depth to see a large change in the vergence angle. It is unknown at this point if a instant change in the vergence angle would have a negative impact on users. But we suspect that an instant change in the virtual camera rotation ( see the differences between Figures 3.3 and 3.4) would cause a conflict with a user's expectation and would thus cause some discomfort (as discussed in Section 2.2.2). Thus we need to create some possible solutions that may help users with this instant change.

The proposed solution for handling an instant change in the vergence angle is to have a transition between the angles. This transition will be done by interpolating between the old and new vergence angles over a period of time such that the change is no longer instant. There are two types of interpolation we will be testing alongside the default case. The first type was linear interpolation as one of the more basic types of interpolation to test. The second was a custom made interpolation we called 'Ease Interpolation', with the goal of seeing if a different type of interpolation had a noticeable effect on users. Thus in total there are 3 different types of interpolation that will be tested:

- No Interpolation

- Linear Interpolation

- Ease Interpolation 
In order to determine the length of time a transition between the vergence angle will take, we will base it off of the behaviour of the human visual system. The average angular speed of the vergence movement by the human eye is approximately 20-25 degrees/second [45]. Thus the time a transition will take will depend on the size of the change in vergence angle. Ease Interpolation uses a beizer curve for an ease-in and an easeout interpolation. The arguments to make this beizer curve were: $[0,0]$ $[0.5,0][0.5,1][1,1]$. In theory the use of a smoother curve will make for a smoother transition.

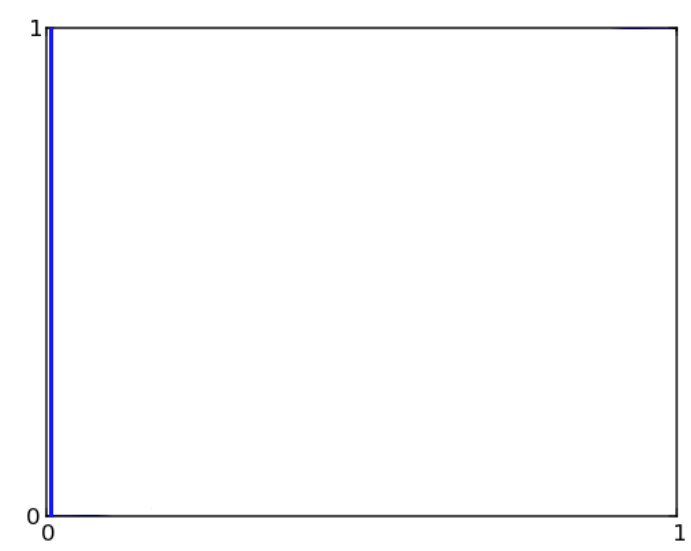

Figure 3.5: No Interpolation

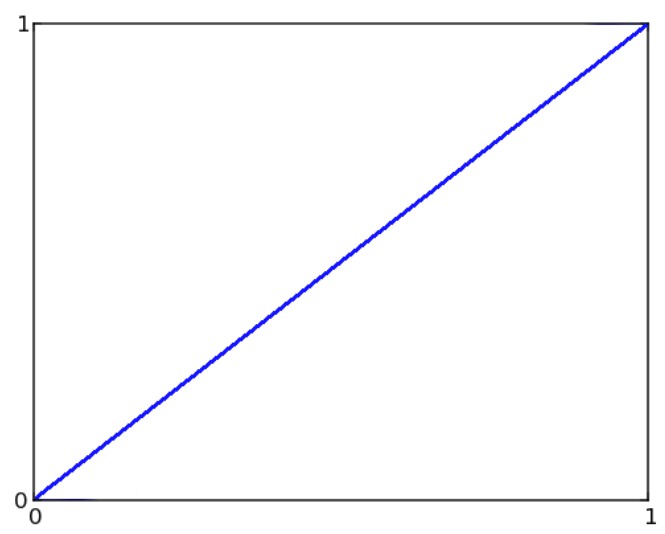

Figure 3.6: Linear Interpolation 


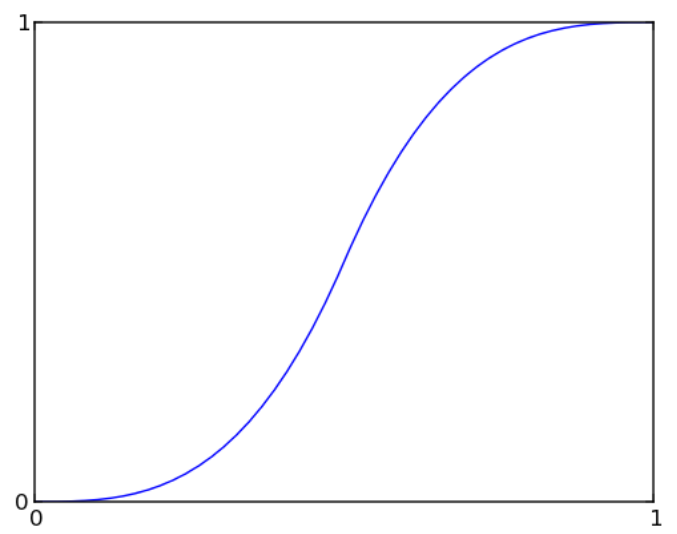

Figure 3.7: Ease Interpolation 


\section{Chapter 4}

\section{Software Implementation}

In the previous chapter, the framework of the DC system and its core algorithms were described and explained. This chapter details the implementation of this system in a commercial game engine, Unity3D. The source code that this chapter references can be found in Appendix A.

\subsection{Unity3D - Game Engine}

For this research, the execution speed took a priority over visual quality. Thus Unity was chosen over other similar game engines such as Unreal Engine 4 (UE4). In addition, Unity3D was selected to implement DC in, for the following reasons:

- Unity is primarily a game engine. This means it is optimised for creating virtual environments, which is ideal for evaluating DC.

- Support for Oculus Rift DK2. 
- Scripts allow for a vast amount of flexibility in system and algorithm construction.

\subsubsection{Unity and Scripts}

Unity uses GameObjects inside its scenes, which can be visible objects inside the game environment or invisible objects with a management role for the scene. These different types of behaviours are dictated by the scripts that are attached to them. The scripts themselves are written in either C\# or Javascript, and uses Unity's own API. These scripts make up the entirety of the DC system itself and the logic behind the scenes used to test the effectiveness of the system.

The UnityEngine API the scripts use have two commonly utilised functions, Start() and Update(). Start() executes once when the scene is first ran, thus it is mainly used for one time setup of variables and functionality. The Update() function executes every frame in the scene, therefore any functionality that needs to be constantly updated is done inside Update().

\subsection{Overview}

The code discussed in this section is located in the Appendix. Figure A.1 shows the Update() function in the script OVRCameraRig.cs which was originally part of Oculus Utilities for Unity (version 1.3.2), but was modified for this implementation. The code of the Update() function encapsulated by the red box in Figure A.1 is what was added to the original script and acts as an overview for the DC implementation. The IF statement chain acts as a manager for settings that can be changed at run-time for the 
purposes of testing. These settings include; the different types of rotational interpolation that were mentioned previously in Section 3.4, whether DC is enabled or disabled via the boolean vergOn and the option of adjusting an offset for the distance between the virtual cameras for the purposes of IPD adjustment during a test. The rest of the functions shown will be explained later in this chapter.

\subsection{Implementing Gaze Approximation}

In order to implement gaze estimation, the position and orientation of the virtual camera in the scene must be known, so that the system can then cast the ray box to find the depth of the object of interest. Both the position and orientation of the camera can be queried using Unity's API. Also as previously mentioned, Unity has support for the Oculus Rift such that the virtual cameras in the scene move according to the relative position and orientation of the HMD itself. With this we are easily able to continuously obtain the position and orientation of the virtual cameras.

Figure A.2 shows the CalculateGazeDist() function which calculates and returns the distance of the current object of interest using the assumptions previously mentioned in Section 3.2 and Algorithm 1.

First the Raybox previously mentioned in Section 3.2.1 is constructed as a List of floats called distRays, these floats are the depths returned from the Raytrace() function shown in Figure A.4, which returns the depth from ray intersection. Most of the code in Raytrace() is from the UnityEngine API. These rays are fired based on the position of centreEyeAnchor which is a gameObject that updates its position with respect to the midpoint between the eyes in the Unity scene using the tracking data from the Oculus Rift. The 'EyeAnchor' gameObjects are part of the 
original script in the Oculus Utilities for Unity (version 1.3.2).

Next, Algorithm 1 is implemented as part of the CalculateGazeDist() function. Figure A.3 shows the smaller functions that are used as part of Algorithm 1. DistCheck() returns true if there exists a depth value that is outside 2 standard deviations and returns false if not. MeanDist() and SSDist() calculate the mean and standard deviation of the current list of depth values respectively.

\subsection{Implementing Dynamic Convergence}

Figure A.5 shows RayGaze() the main function that handles the virtual camera rotation which is called from Update(). First the distance between the virtual cameras is obtained, eyeDist, by getting the distance between the left and right EyeAnchor gameObjects, then the offset mentioned in the Update() function is applied. The vergence depth is then obtained from the previously explained function CalculateGazeDist(), and stored in the variable hitDist. The IF statement and the DrawRay code that follows was to help discern whether the user was estimated to be looking at something in the foreground or the background, this was used in the user evaluation in Chapter 5.

As long as the vergence depth was not zero (i.e. infinity) and there was no interpolation currently occurring with the right or left virtual cameras, a new vergence angle is calculated using Equation 3.1. Then, the axis upon which to rotate the virtual cameras must be found, which is done by obtaining the eyeAxis which is the axis on which both virtual cameras sit on which changes as the HMD's position and orientation changes. This is accomplished by querying the position of leftEyeAnchor and rightEyeAnchor, these are gameObjects that update position with re- 
spect to the left and right eyes.

Once the eyeAxis is obtained the rotationAxis, the axis upon which to rotate the virtual cameras about, is found by taking the cross product of the eyeAxis and the forward direction the HMD is facing, which is done by querying the forward direction of centreEyeAnchor. Using the rotational axis and the angle of vergence, a quarternion is obtained and multiplied to the virtual cameras' rotation, these are recorded as the new rotations for each camera, the directions of each rotation are changed appropriately for the respective camera.

If the vergence depth was zero, then the new vergence angle is at infinity, thus the vergence angle is set to zero and the new rotation is the virtual cameras' default rotation if it wasn't already set at default.

With the vergence angle and the corresponding new camera rotation calculated for each eye, the old vergence angle and camera rotations as well as the current time are recorded for the interpolation function to use, then the interpolation for each eye is enabled using a boolean.

To actually rotate the virtual cameras, a parent-child relationship was set up in the scene's hierarchy as modification to the original Unity prefab found in the Oculus Utilities for Unity (version 1.3.2). Figure A.6 shows this setup. In Unity, applying a rotation at run-time to a camera that follows the position and orientation of the Oculus Rift has no effect as that rotation just gets overwritten by said position and orientation.

This setup bypasses this problem by applying a translation to the parent (LeftEye and RightEye) such that the children (LeftCam and RightCam, the gameObjects with the camera components attached) are at the same position as centreEyeAnchor before any vergence rotation is applied. This is done via the UpdateParent() function shown in Figure A.7 which is called once for eye camera in the Update() function. The orientation of 
the cameras are already correct as they follow the same orientation as the Oculus Rift during run-time as part of the Oculus Rift's integration with Unity. Then, any vergence rotations are applied to the respective parents in order to correctly rotate the cameras.

The camera component on each of LeftCam and RightCam can be seen in Figure A.8, their only difference being only outputting to their respected eye. The reason why the virtual cameras follow centreEyeAnchor and not the leftEyeAnchor or rightEyeAnchor respectively is that for one camera inside Unity, the Oculus Utilities for Unity already take the IPD into consideration when displaying to each eye. If the virtual cameras were to follow the leftEyeAnchor and rightEyeAnchor respectively the IPD would be essentially doubled as it would be taken into account twice, which is incorrect.

\subsection{Implementing Vergence Interpolation}

Figure A.9 shows the LerpCheck() function which is called once for each eye in Update(). LerpCheck() takes in global variables as arguments that were given values in RayGaze(), these variables are global because the information for interpolation must persist for multiple Update() calls for the interpolation to finish. There is a global variable version of each LerpCheck() argument for each eye.

The first argument is a boolean lerping that enables the interpolation. While the interpolation is enabled no other interpolation related variable is changed via RayGaze(). The lerping variable is disabled upon the completion of the current interpolation. Arguments aRot and bRot are the old and new vergence rotations respectively, while the argument target is the gameObject the rotation is to be applied to (LeftEye or RightEye). 
The argument startTime, which is the time at which the interpolation started. The time the interpolation takes to complete depends on the difference between the old and new vergence angle which is given in the last argument angleChange. For this implementation, the angular speed of the vergence movement was chosen to be 25 degrees/second based on a previously mentioned approximation. This angular speed is used to calculate the overall time the interpolation takes. For optimization reasons, if the change in vergence angle is very small (less than 0.1 degree) then the change happens instantly without any interpolation as such changes are barely noticeable. By subtracting the current time from startTime and then dividing by the overall time, deltaTime is given which is the percentage of completion a linear interpolation should be, at the current frame.

As discussed in Section 3.4, there were three different types of interpolation which can be changed at run-time via input in Update() mentioned in Section 4.2. While the global variable 'interpolation' is false, the transition 'No Interpolation' is active. While the global variable 'useCurve' is true, the transition Ease Interpolation is active. If 'interpolation' is true and 'useCurve' is false, the transition Linear Interpolation is active. If 'No Interpolation' is active the vergence rotation is just set the new vergence rotation and no interpolation happens. While in the case of Linear Interpolation, deltaTime is calculated as described previously and Unity's Lerp() function is used to do the interpolation. Lastly, if Ease Interpolation is active deltaTime is still calculated but given to a function that returns the position it would have on a bezier curve (seen in Figure A.10) the function was designed with 3D space in mind, so the arguments given to the bezier curve mentioned in Section 3.4 were modified such that they simply all lay on the x-y plane: $[0,0,0][0.5,0,0][0.5,1,0][1,1,0]$. The function returns the position that the percentage of completion would have on this curve, the y-coordinate is then given back to Unity's Lerp() to handle the interpolation. 


\section{Chapter 5}

\section{A Quantitative Experiment on Dynamic Convergence}

As part of the investigation of DC, a user test is carried out to measure the difficulty of performing binocular fusion experienced by participants, as well as monitor any side-effects in the form of discomfort symptoms. This was done for both stationary and instantaneously moving close virtual objects. From this data we hope to find an approximate vergence depth range that DC is most effective at aiding in binocular fusion, and if changes in vergence depth have an impact on the range. In addition, this experiment also aims to determine if there is a need for a transition between different vergence angles. To this end, the severity of discomfort symptoms and binocular fusion difficulty are measured for any potential positive or negative effects from 3 different types of interpolation. Afterwards, participants were questioned in a short interview to find their preference of interpolation.

This chapter details the process of the performed experiment, the virtual test scenes and the metrics used as well as the statistical techniques 
used in the analysis of the results.

\subsection{The User Test}

The user test was split into two sessions. Session 1 tested two different cases, in which participants viewed close virtual objects that are either stationary (part 1) or moved instantaneously (part 2). These virtual objects were placed at or moved to a variety of different distances. The tests were repeated with DC enabled and DC disabled.

Session 2 tested the need for a transition between vergence depths. Participants looked at two different virtual objects at a combination of different distances, for each of 3 different types of interpolation:

1. No Interpolation

2. Linear Interpolation

3. Ease Interpolation

\subsubsection{Setup}

\section{Participants}

In order to find participants for this study, posters advertising the study were placed around the Victoria University campus, the poster used is shown in the Appendix B.1. Potential participants were dismissed from the study if they:

- Had any severe eye abnormalities (excluding being short/long sighted). 
- Were pregnant.

- Were predisposed to motion sickness.

This was done because it was believed that effects from these conditions would have a significant impact on the results of the study, that would obscure the investigation of DC. All participants completed a consent form to confirm they understood the risks and processes of the study (B.3). No compensation was offered for participation in the study, as the appeal of using the Oculus Rift was considered attraction enough. Although, out of appreciation, a short VE was shown at the end of the experiment. Ethics approval was granted by the Victoria University Human Ethics Committee. The advertisements resulted in a total of 22 participants for the study. From the 22 participants, 4 did not complete the study and 3 were excluded for varying reasons. This left 15 participants with ages ranging from 17 to 36 years old. 7 participants were female and 8 were male, and all participants were current or prior students of Victoria University of Wellington. The age range does introduce a limitation: visual performance is known to change with age, so the findings of this study are mainly relevant to young adults only, and may not apply other ages groups.

\section{Testing Hardware}

Participants completed the study on a machine running Windows 7 with 8GB RAM, a 3.5GHz Intel octocore CPU and a NVIDIA GTX 970 GPU with an attached Oculus Rift DK2 HMD. This machine was powerful enough to reliably achieve acceptable frame rates for the use of HMDs when running the test scenes during the evaluation, as well as being at a reasonable price point, thus being a reasonable approximation of consumer hardware. 


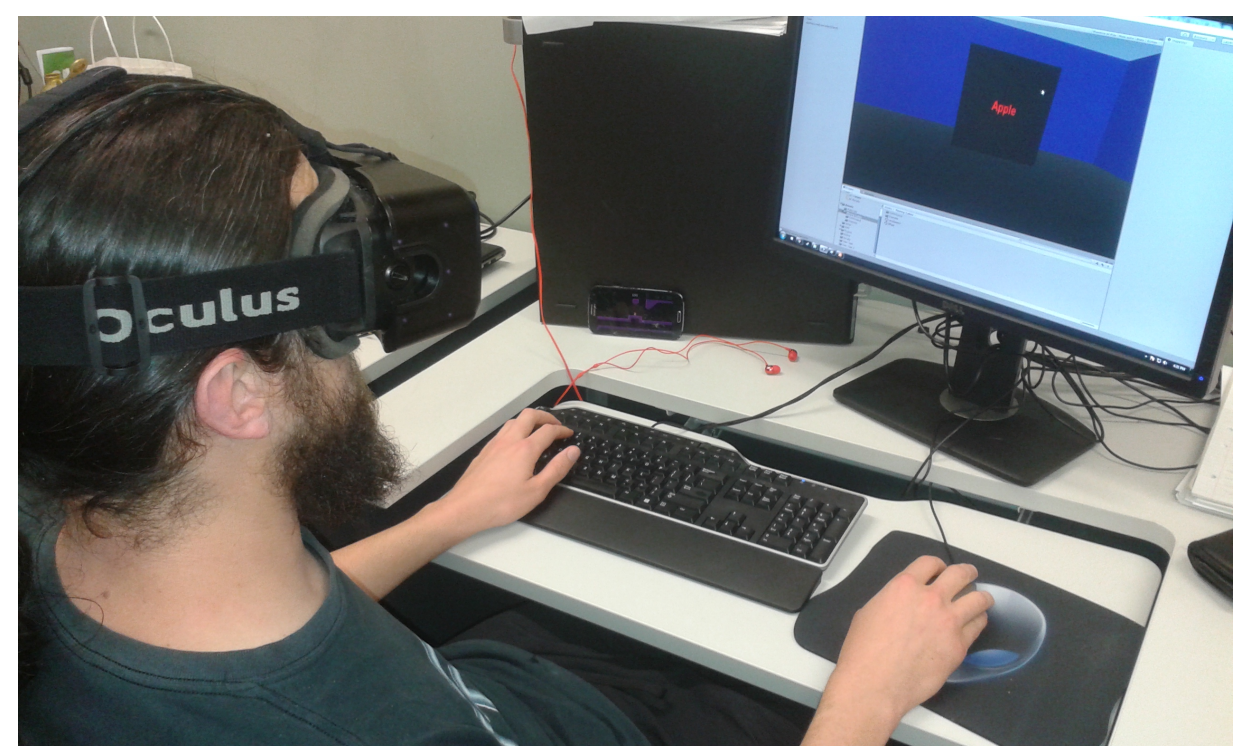

Figure 5.1: Shows the study setup for a participant

\subsubsection{Procedure}

The test scenes used in the evaluation were kept simple to help isolate any unforeseen variables that may have an unknown effect on the investigation. Thus the test scenes only contained a small number of virtual objects (1 or 2) with some detail to focus on ( for example, the word "Apple"), with a very basic background the so the user could have something else look at that also served as a reference point for virtual camera rotation.

For calibration, an approximation of the user's IPD was taken using a ruler, with the option of making further adjustments during the evaluation as needed. The evaluation involved two sessions which took place on separate days for each participant. Throughout the evaluation participants were asked a set of questions, on a likert scale of $1-5$ to rate different discomfort symptom severity and how difficult it was to perform binocular fusion. There were seven different questions asked (the exact wording of these questions can be found on the questionnaires in Appendix B): 
- General Discomfort

- Fatigue

- Headache

- Eyestrain

- Nausea

- Dizziness

- Difficulty Fusing

For the symptoms of discomfort, a 1 on the likert scale meant no severity of that symptom was experienced and a 5 on the scale meant very high severity was experienced. For 'Fusion Difficulty' a 1 on the likert scale meant that the participant found it easy to perform binocular fusion, while a 5 on the scale meant that the participant found it impossible to perform binocular fusion and thus saw double vision. The questionnaires used for this evaluation can be found in Appendix B.

\subsubsection{Session One}

The goal of the first session was to:

- Investigate how effective DC was at aiding users with fusing stationary close virtual objects.

- Observe how effective DC was at aiding users with fusing at instantly changing vergence depths.

- Measure any effects that DC has on symptoms of discomfort. 
Session one was ran in two parts. Part one focused on the stationary close objects, while part two focused on the instant vergence depth changes. In each part a test involving multiple scenes was conducted. The same tests were done once with and without DC, the order of which was randomised.
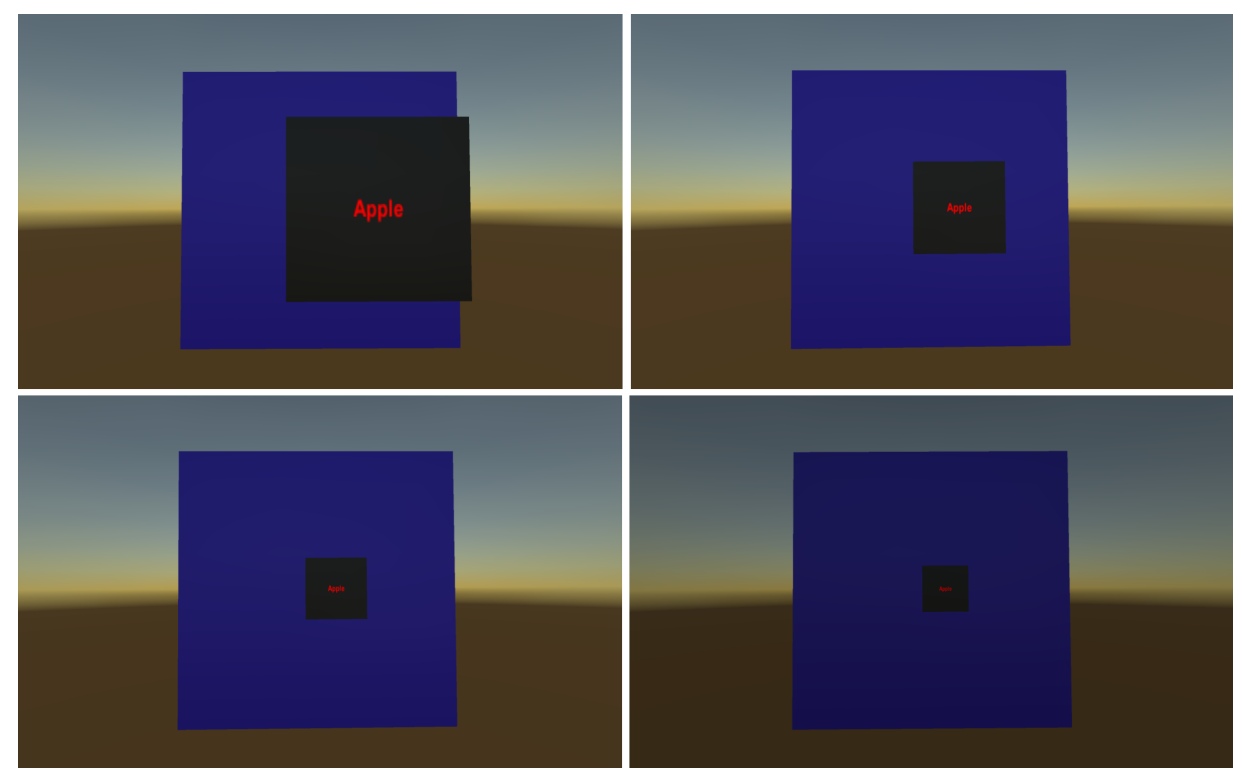

Figure 5.2: The scene used in session one, which featured the same virtual object at a vergence depth of $0.15 \mathrm{~m}, 0.30 \mathrm{~m}, 0.45 \mathrm{~m}$ and $0.60 \mathrm{~m}$ respectively.

For part one of the first session, the set of questions were asked before and after all the scenes and once during each of four scenes. Each scene had a cube at a certain distance in front of the user with some text written on it (Figure 5.2). The distance changed from each scene such that $0.15 \mathrm{~m}, 0.30 \mathrm{~m}, 0.45 \mathrm{~m}$ and $0.6 \mathrm{~m}$ distances were all tested once each. After answering the set of questions for a scene the screen faded out to black for the next scene to reset the user's depth cues.

For part two of the first session, the set of questions were asked once during each of six cases. Each case had the same cube from part one but the cube would periodically and instantly change between two distances from the user. The distance scenarios are shown in Figure 5.4. 


\begin{tabular}{|l|l|l|l|l|l|l|}
\hline & Pre & 0.15 & 0.3 & 0.45 & 0.6 & Post \\
\hline General discomfort & & & & & & \\
\hline Fatigue & & & & & & \\
\hline Headache & & & & & & \\
\hline Eyestrain & & & & & & \\
\hline Nausea & & & & & & \\
\hline Dizziness & & & & & & \\
\hline Difficulty fusing & & & & & & \\
\hline
\end{tabular}

Figure 5.3: An excerpt of the questionnaire used in session one part one (see Figures B.4 and B.5 for the full questionnaire). The columns list the distances used. The rows are the symptoms participants were asked to rate on a likert scale of 1-5 in terms of severity.

\begin{tabular}{|c|c|c|c|c|c|c|}
\hline & $0.15->0.3$ & $0.15>0.45$ & $0.15->0.6$ & $0.3->0.45$ & $0.3->0.6$ & $0.45->0.6$ \\
\hline General discomf & & & & & & \\
\hline Fatigue & & & & & & \\
\hline Headache & & & & & & \\
\hline Eyestrain & & & & & & \\
\hline Nausea & & & & & & \\
\hline Dizziness & & & & & & \\
\hline Difficulty fusing & & & & & & \\
\hline
\end{tabular}

Figure 5.4: An excerpt of the questionnaire used in session one part two (see Figures B.4 and B.5 for the full questionnaire). The columns list the distance cases used. The rows are the symptoms participants were asked to rate on a likert scale of 1-5 in terms of severity. 


\subsubsection{Session Two}

The goal of the second session was to investigate the need of a transition between vergence depths. Which involved testing three different types of interpolation:

1. No Interpolation

2. Linear Interpolation

3. Ease Interpolation

These were tested for their effects on visual discomfort and binocular fusion. This session also included a short interview in order to find what type of interpolation users preferred and why.

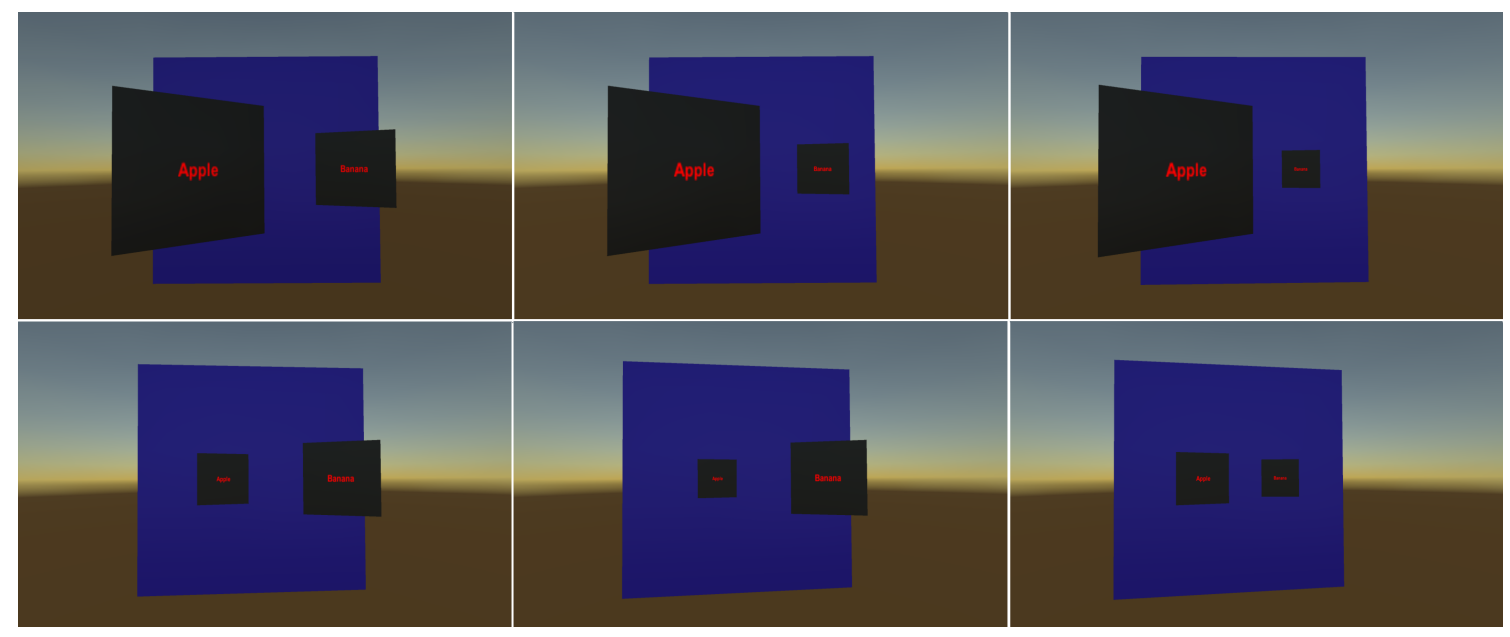

Figure 5.5: The scenes used in session two (in order).

The same set of questions from before were asked before and after all the scenes, as well as once during each of six scenes. Each scene had two cubes with different text on them in front of the user at different distances (Figure 5.5). The participant was asked to look from one cube to 
the other repeatedly at their own pace by moving their head. The distance pairs are shown in Figure 5.6. This was repeated for each of the 3 types of interpolation, the order of which was randomised.

After answering the set of questions for a scene the screen faded out and in from black for the next scene to reset the user's depth cues. At the end of all three tests, the participant was then given the ability to switch between the different types of interpolation (only referred to as " $\mathrm{A}$ ", "B" and " $\mathrm{C}$ ") for one previously shown scene and in a small interview was asked which type they preferred, why they preferred it, and how it compared against the other types of interpolation. This interview was conducted because of the possibility that having a purely quantitative set of results may not fully reflect what the most ideal type of transition is.

\begin{tabular}{|c|c|c|c|c|c|c|}
\hline Distances & $0.15->0.3$ & $0.15->0.45$ & $0.15->0.6$ & $0.3->0.45$ & $0.3->0.6$ & $0.45->0.6$ \\
\hline Key & 1 & 2 & 3 & 4 & 5 & 6 \\
\hline
\end{tabular}

\begin{tabular}{|l|l|l|l|l|l|l|l|l|}
\hline & Pre & $\mathbf{1}$ & $\mathbf{2}$ & $\mathbf{3}$ & $\mathbf{4}$ & $\mathbf{5}$ & $\mathbf{6}$ & Post \\
\hline General discomfort & & & & & & & & \\
\hline Fatigue & & & & & & & & \\
\hline Headache & & & & & & & & \\
\hline Eyestrain & & & & & & & & \\
\hline Nausea & & & & & & & & \\
\hline Dizziness & & & & & & & & \\
\hline Difficulty fusing & & & & & & & & \\
\hline
\end{tabular}

Figure 5.6: An excerpt of the questionnaire used in session two (see Figures B.6 and B.7 for the full questionnaire). The columns list the distance scenarios used. The rows are the symptoms participants were asked to rate on a likert scale of 1-5 in terms of severity. 


\subsection{Analysis}

In this section, the results from both the quantitative and qualitative findings of the user test are introduced and analysed using a variety of statistical techniques including the use of the IBM computing software: Statistical Package for the Social Sciences (SPSS) Version 24 for the analysis of the quantitative results. This largely consists of comparing the results of symptom severity or fusion difficulty scores across the different experiment treatments.

The experimental data obtained from the user test sample population is analysed and used in order to deduce statistical inferences using well researched statistical techniques so that some useful estimates of likely characteristics for the broader population may be derived. It is however, important to point out that the "statistical significance" referred to throughout the following sections relates to the mathematical probability of obtaining the observed results if these same experiment were to be carried out many times across more samples of participants in the broader population, it is not to be confused with practical significance.

The analysis here will adopt the same common statistical inference principles as those used in physiological and social science research [6], where it is common practice to state a null hypothesis as such that there is "no effect", which in this case means there is no difference in the symptom severity (or fusion difficulty) scores between the respective experiments or treatment groups being compared. We shall then look at the strength of statistical evidence to reject the null hypothesis in favour of accepting the alternative hypothesis, that there is an effect (or difference). It will also be sufficient to adopt the commonly used significance level of $P$ value at 0.05 , which denotes a 5\% probability or 1 in 20 chance of being wrong, based on assumption that the null hypothesis is true. 
What this means is that if the P value is less than 0.05 then the null hypothesis can be safely rejected i.e. accepting that the survey sample gives reasonable evidence to support the alternative hypothesis, and the fact that there are statistically significant differences in the scores being compared. It does not necessary imply that it is an important or practical difference, this needs to be considered in the context of the overall practical relevance and results which will be discussed in further chapters. What it means is that this is the probability of obtaining a result (difference in fusion difficulty scores) as large or greater than this if no true effect existed in the population. For example, if the statistical test revealed a $\mathrm{P}$ value of 0.05 and if there really was no true differences between the treatments (null hypothesis is true), we would observe this result in 5 out of 100 repeated experiments, or 1 in 20 occasions. Therefore this challenges the assumption that the null hypothesis is true, and provides support for the alternative hypothesis that there is a difference between treatments and therefore we can say that the observed resultant difference is statistically significant.

\subsubsection{Statistical Methods}

It is important that the correct statistical methods are used so as to be able to make valid and defendable statistical inferences from the findings.

Typically survey results such as those captured from this research produce ordinal data (responses from a likert scale, 5-point rating, etc.) and these do not have a true mean or standard deviation since it can not be assumed that the distance between responses is equidistant. The choice of statistical tests for the most part appear to be largely dependent on the resulting raw data that has been collected. For example the number of cases and whether the data-set represents a normal distribution. This leads to the selection of either parametric or non-parametric tests, the latter being 
the most suitable for when the data violates the rules of normal distribution such as:

1. Variables are measured on a nominal or ordinal scale.

2. The data violates the normality assumption.

3. The sample size is small (less than 20 cases or subjects).

Moreover, the selection of a robust statistical test is also recommend as it will perform adequately even if some of its assumptions are moderately violated. In this respect, non-parametric tests are arguably more robust than their parametric counterparts, in particular when dealing with very small sample sizes and where the data is not normally distributed. The flowchart below provides a useful reminder in the selection of the correct statistical tests (figure 5.7).

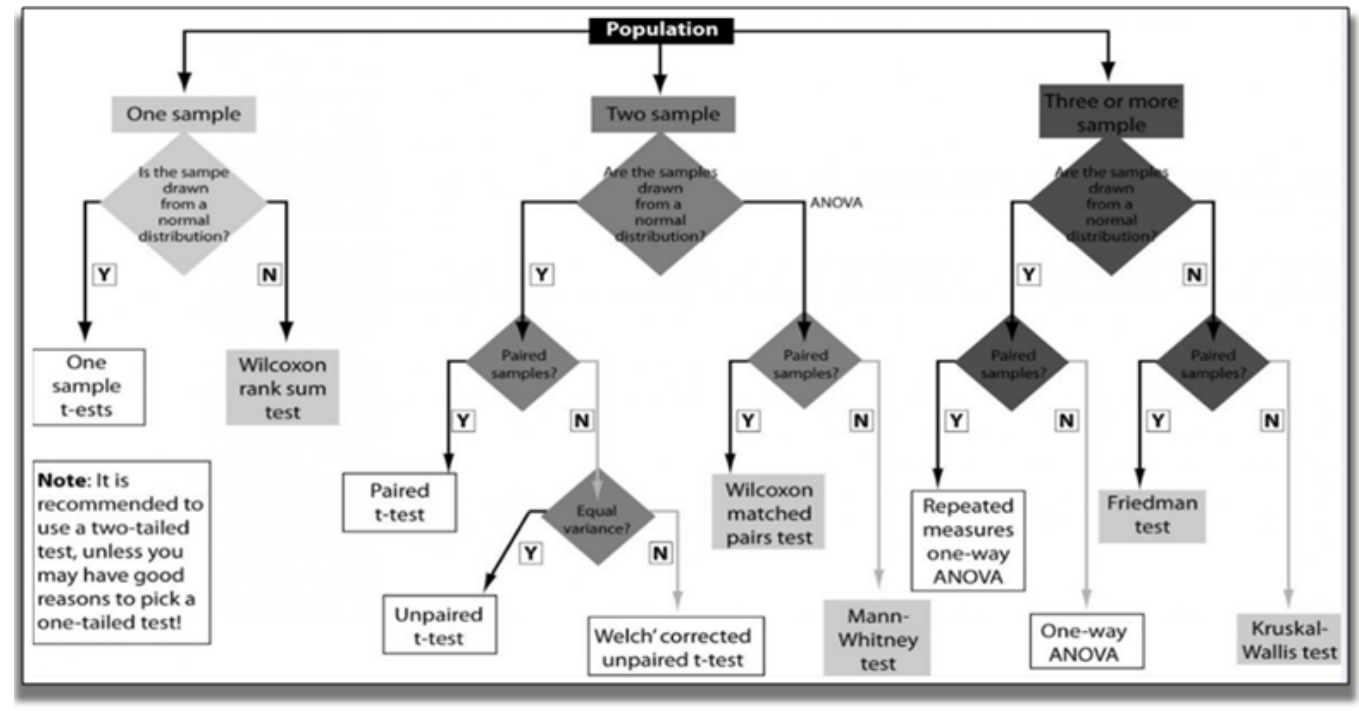

Figure 5.7: Selection of the correct statistical tests [8]

In this research since the data collected is a combination of two or more pairwise (related) samples that contain ordinal data which will not necessarily meet the assumption of being drawn from a normal distribu- 
tion. It can be seen from Figure 5.7 that the best choice of initial statistical tests will be a combination of the Friedman test (where there are three or more sets of observations being compared) and the Wilcoxon matched pairs test (aka Wilcoxon signed rank test) where there are two sets of observations being compared. These statistical test techniques where chosen since much of the analysis involved comparing the questionnaire scores (discomfort and ease of fusion difficulty) across numerous different treatment conditions (test scenes and changing viewing distances).

\section{The Friedman Test}

The Friedman test is a non-parametric statistical test developed by the American Nobel Prize winning economist Milton Friedman, it is used to detect differences in treatments across three or more multiple related test attempts. The Friedman test, unlike its parametric counterparts where numerical scores can be used to compute means and variances, work instead with categorical and ordinal data. It utilizes the chi-square $\left(\mathrm{X}^{2}\right)$ statistic which in turn determines whether distributions of categorical variables differ from one another.

The procedural calculation of the Friedman test involves converting the ordinal values of interest (test scene scores from the questionnaires) into ranks and then computing the sum of these ranks for each treatment condition. It also requires the number of participants in the sample $(\mathrm{N})$ and the number of treatment conditions $(\mathrm{K})$, the latter being the 4 or 6 different viewing scenes.

The Friedman test then evaluates the differences between the respective treatments by computing the chi-square test statistic for ranks. This test statistic has degrees of freedom that are determined by $d f=k-$ 1 , where $k$ equates to the number treatments. By establishing a signifi- 
cance level, such as the commonly used $\mathrm{p}$ value $<0.05$ (adequate for this research), the results are evaluated against a hypothesis by comparing the results with the critical value in a chi-square distribution table as shown in Figure 5.8. However, this is shown just for reference as all of the calculations have been done by the statistical analysis software program SPSS.

The generally accepted null hypothesis for statistical tests such as the Friedman test is to state that there are no systematic or consistent differences between the treatment conditions being compared. That is that the ranks in one treatment should not be systematically higher or lower than the ranks in any other treatment condition.

H0: - There is no difference in discomfort (or difficulty) scores between each of the test conditions.

H1: There are differences in discomfort (or difficulty) scores between each of the test conditions.

The calculated chi-square result must be greater than the critical value for chi-square (from look up table) in order to reject $\mathrm{H} 0$ and conclude that there are significant differences among the treatments.

\begin{tabular}{|ccc|}
\hline DF & $P=0.10$ & $P=0.05$ \\
\hline 1 & 2.706 & 3.841 \\
2 & 4.605 & 5.991 \\
3 & 6.251 & 7.815 \\
4 & 7.779 & 9.488 \\
5 & 9.236 & 11.070 \\
& & \\
6 & 10.645 & 12.592 \\
7 & 12.017 & 14.067 \\
8 & 13.362 & 15.507 \\
9 & 14.684 & 16.919 \\
10 & 15.987 & 18.307 \\
\hline
\end{tabular}

Figure 5.8: Shows a section of the the relevant Chi-Square critical values that will used by the Friedman Test in this analysis. 


\section{The Wilcoxon Signed Rank Test}

The Wilcoxon Signed Rank Test is another non-parametric statistical test ideal for use with repeated measures of the same related subjects. It is the non-parametric equivalent to the repeated measures t-test, but instead of comparing means,in order to obtain an approximate idea of the significance of the differences it converts all the scores to ranks and compares them across the different treatment conditions. It is used here for pairwise post hoc analysis following the Friedman Test since the latter only identifies that there are differences between the ranked means of multiple groups and does not specify exactly which pairs of results differ with each other.

SPSS, as like most other statistical software packages, conveniently performs approximate Z-tests which has +/-1.96 as its critical statistic values for $\mathrm{p}=0.05$ (for a two tailed test).

It is also worth noting that there is a need to perform a simple manual calculation when performing post hoc tests such as these, this is known as a Bonferroni adjustment since making multiple comparisons makes it more likely that a statistically significant result could be declared when it should not be (this is known as a Type I error). The Bonferroni adjustment is done by dividing the original significance level (0.05) by the number of comparison tests. For example, if we compare three pairs we will have a new significance level of $0.05 / 3=0.017$. Therefore, if the result is larger than the new $\mathrm{p}$ value of 0.017 , then we do not have a statistically significant result. 


\subsubsection{Invalid Results}

As mentioned in the previous chapter, there were originally a total of 22 participants in the population sample for this research. From these, 4 did not complete the experiments and 3 were discarded due to being biased or invalid results. These results included:

A participant that caused the assumption of 'a user will look at the centre region of the screen' to become invalid due to abnormal head posture. Prompts to compensate for this proved unsuccessful, thus the result was discarded.

Another participant recorded their pre-test symptoms of fatigue at severity of 5 and a headache at severity of 3 . In the first session while DC was disabled during part 1 of the test, the participant reported that "the box was moving and the colours were bleeding into each other". Due to the high pre-test symptom severity and the abnormal report the experiment was ended early and the result was discarded due to possible bias.

The final discarded result was a participant of age 66 who was prescribed bifocal glasses. Due to the constant use of bifocal glasses the estimated gaze depth initially didn't work due to abnormal head posture. Though after a prompt to correct the head posture it was discovered that DC actually had quite a negative impact on fusion difficulty, unlike any other previously recorded result. It is unknown if the cause was the large difference in age or the use of bifocal glasses. While the advertisement for this study stated that participants with any severe eye abnormalities (excluding being short/long sighted) would be dismissed, the use of bifocal glasses did not seem severe enough to initially dismiss the participant. Investigating the effects that DC has on a large range of ages and eye conditions was deemed outside the scope of this research topic and thus the result was discarded. 
Therefore, for the first part of the experiment there was a total sample population of 15 valid participants almost evenly split across gender (8 males and 7 females).

\subsubsection{Analysis of Session 1 Part 1 - Static Virtual Object}

As described in Section 5.1.3, participants looked at a close stationary object positioned at different distances away from them. The data from this part of the evaluation was entered into both Excel and SPSS for initial exploratory analysis, followed by more detailed statistical analysis. This section illustrates the findings. As mentioned earlier due to the nature of the sample population and the ordinal data results using means and averages to summarise is not appropriate, therefore, median scores and statistical techniques using ranking is used instead. Figure 5.9 illustrates the first observation, namely the impact of DC, more specifically the median discomfort scores for all symptom severities when viewing a stationary close virtual object across four different distances each (including the fusion difficulty test). While several participants gave scores other than ' 1 ' to various test scenarios, the median score for all but one scenario was 1.0. The only notable scenario in which the median differed from 1.0 was for fusion difficulty at $0.15 \mathrm{~m}$ when DC was disabled, where almost all participants reported having a very high difficulty performing binocular fusion fusing.

To obtain a meaningful statistical inference from these results, SPSS was used and the obvious area to focus analysis was the impact of DC across the different viewing distances. Firstly in order to check the statistical significance of the first visual, a hypothesis was derived for the first part of the analysis (i.e. $\mathrm{HO}=$ there is no difference in the ease of fusion difficulty across all four viewing distances of $0.15 \mathrm{~m}-0.6 \mathrm{~m}$ with DC disabled). Using SPSS a Friedman test set up and executed. This compared the participants reported ease of fusion scores across all four viewing dis- 
tance scenarios (with DC disabled) to determine if there was a notable difference.

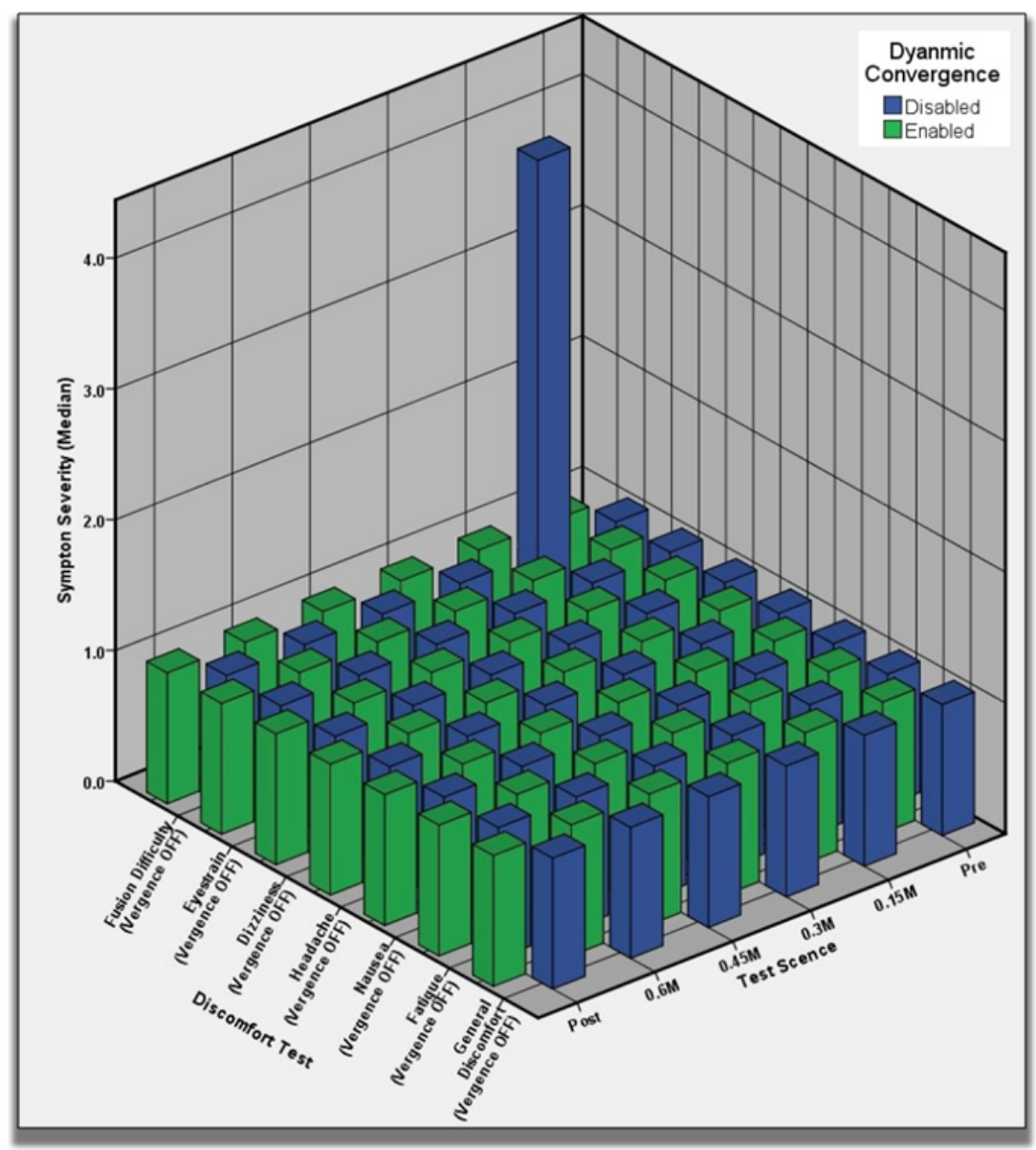

Figure 5.9: Shows the median discomfort scores across all test scenarios

One of the useful values output by SPSS is the Mean Rank, which is indicative of the magnitude of the individual ranked scores for each test treatment. It can be seen by Figure 5.10 (see Ranks table), that the scores for these Mean Ranks for the four different ease of fusion viewing distance suggest a reduction in the ease of fusion difficulty as the distance increases. That is, fusion becomes more difficult at the shorter distance $(0.15 \mathrm{~m})$ and it became notably easier at $0.6 \mathrm{~m}$. 


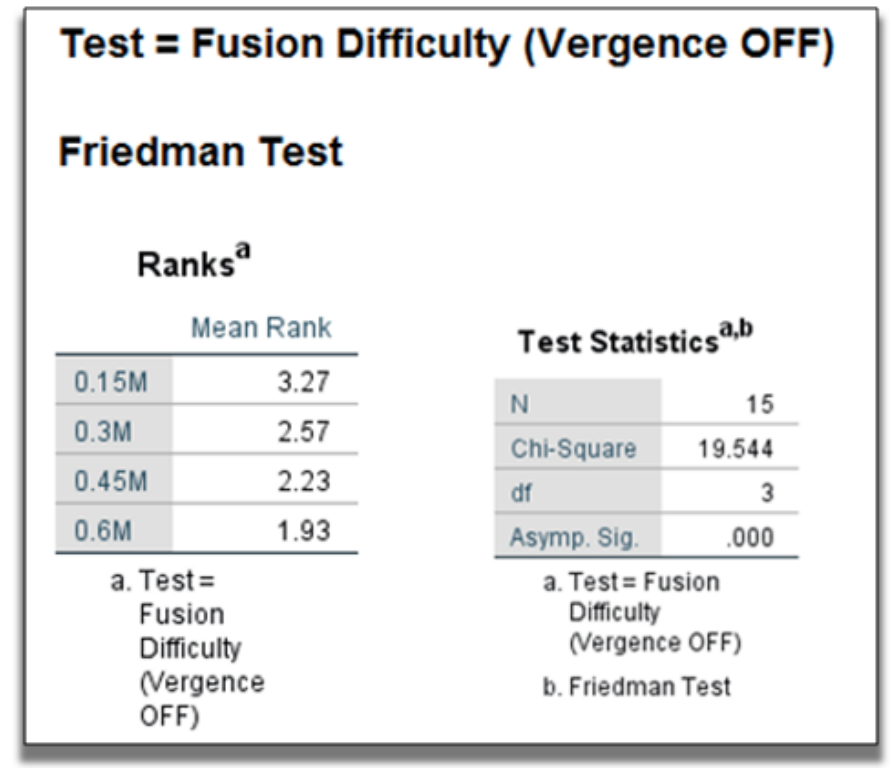

Figure 5.10: Results of the Friedman test

The results of the first Friedman Test (Figure 5.10, Test Statistics table) indicate that there appears to be statistically significant differences between the groups for ease of fusion across all four viewing distances with DC disabled $\left.X^{2}(3, \mathrm{n}=15)=19.544, \mathrm{p}<.05\right)$. As can be seen the calculated chi-square value is greater than the critical value (this can be checked by comparing the critical chi-square value in figure 5.8 , with 3 degrees freedom and $\mathrm{p}$ value $=0.05$ where it can be seen that the critical chi-square value $=7.815$ ). Therefore the null hypothesis $\mathrm{H} 0$ can be rejected and the alternative hypothesis $\mathrm{H} 1$ accepted, that is:

$\mathrm{H} 1$ = There is a difference in fusion difficulty scores across the four test treatments.

The Friedman test, like its parametric alternative is able to confirm that there are differences of statistical significance between the groups, however, it cannot specifically pinpoint the exact group differences. Therefore post hoc analysis is required in order to confirm which resultant groups 
are different from each other along with more detail as to the magnitude of the difference. To do this the Wilcoxon Signed Rank Test was used. The output of which is shown in Figure 5.11.

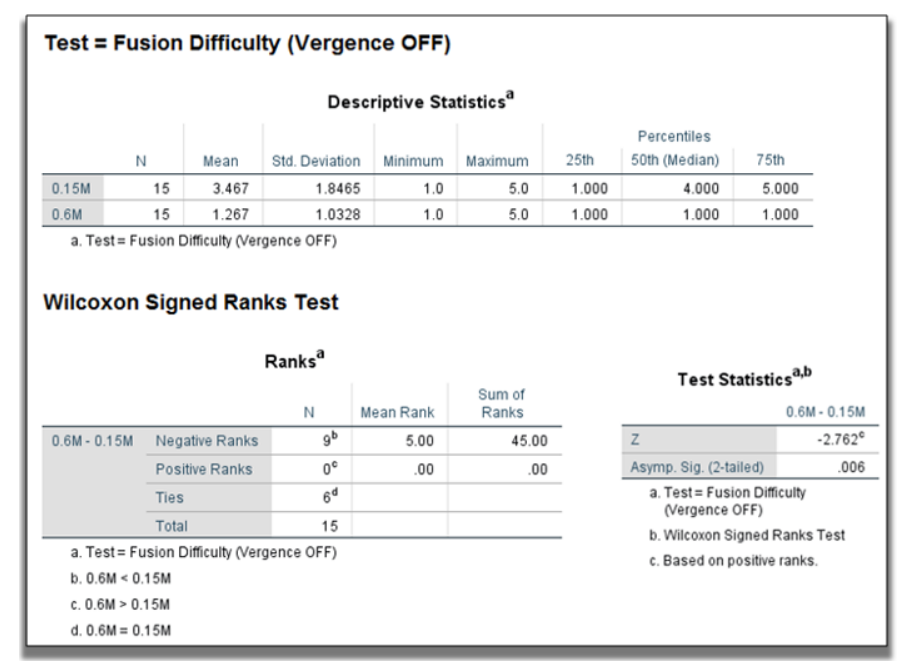

Figure 5.11: Results of the Wilcoxon Signed Rank Test

From the Wilcoxon Signed Rank Test, the most relevant result was shown to be between the shortest $(0.15 \mathrm{~m})$ and the furthest $(0.6 \mathrm{~m})$ viewing distances, as it had the greatest difference. The other results were not as significant. From Figure 5.11 above it can be seen that there is a statically significant difference between the median ease of fusion scores when comparing the distances $0.15 \mathrm{~m}$ and $0.6 \mathrm{~m}$. As shown in the Ranks table "Negative Ranks", the discomfort or ease of fusion scores at $0.6 \mathrm{~m}$ is less than those at $0.15 \mathrm{~m}$ on 9 occasions, meaning 9 participants found binocular fusion easier at the greater distance. There are 6 ties and zero "Positive Ranks" i.e. occasions when $0.6 \mathrm{~m}$ is scored higher than $0.15 \mathrm{~m}$ on the symptom severity scale. Therefore this infers that with DC disabled as the viewing distance increases from $0.15 \mathrm{~m}$ to $0.6 \mathrm{~m}$ the ease of fusion and discomfort decreases (becomes easier). Or alternatively stated, with DC Disabled, binocular fusion is more difficult at shorter distances. 
Therefore a Wilcoxon Signed Rank Test revealed a statistically significant reduction in the ease of binocular fusion score (i.e. viewing became easier) when viewing a stationary object at $0.6 \mathrm{~m}$ compared to that at $0.15 \mathrm{~m}$ with $\mathrm{DC}$ disabled the results are: $Z=-2.762, \mathrm{p}<0.05$. With the Bonferroni adjustment applied (original $\mathrm{p}$ value divided the number of tests: $0.05 / 2$ ) still gives a statistically significant result with the new $p$ value of 0.025 , which is greater than the reported value $p=0.006$ ).

Remaining with fusion difficulty, attention was now turned to comparing the effect on participant's ease of fusion difficulty scores when having DC enabled (versus disabled) across the same four viewing distances $(0.15 \mathrm{~m}$ to $0.6 \mathrm{~m})$ for the same stationary object. Figure 5.12 shows an Excel chart for the ease of binocular fusion difficulty scores of all participants (recalling that $1=$ easy and $5=$ impossible) for DC both enabled and disabled. Initial inspection of the results by eye alone suggests that there may be evidence to support the hypothesis that participants found binocular fusion easier with DC enabled.

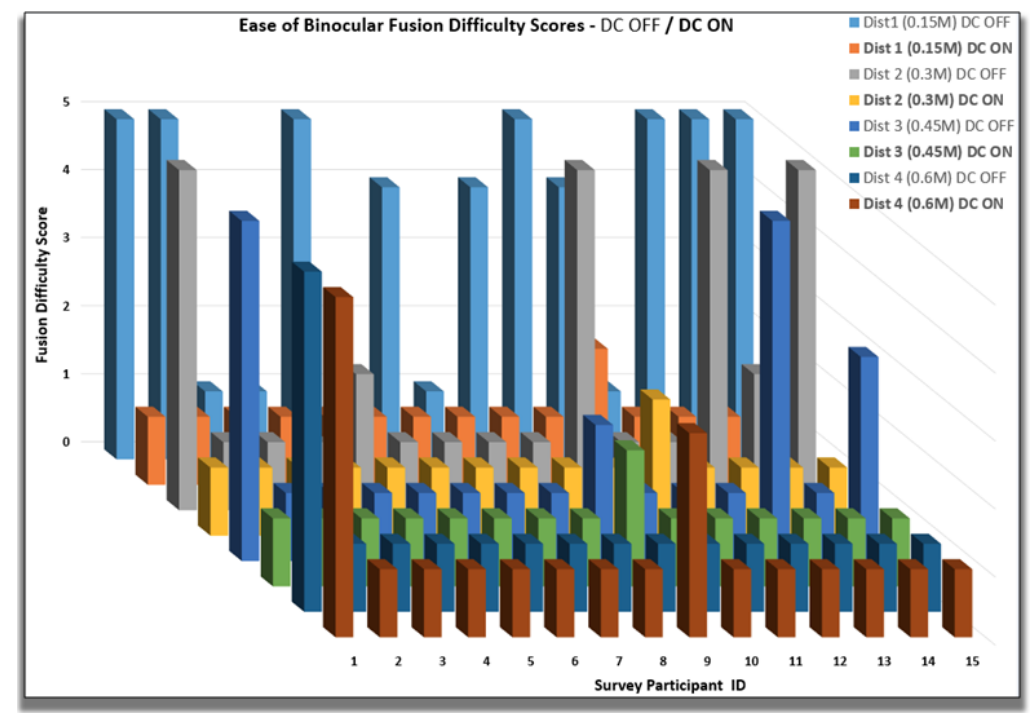

Figure 5.12: Shows binocular fusion difficulty scores for all participants 


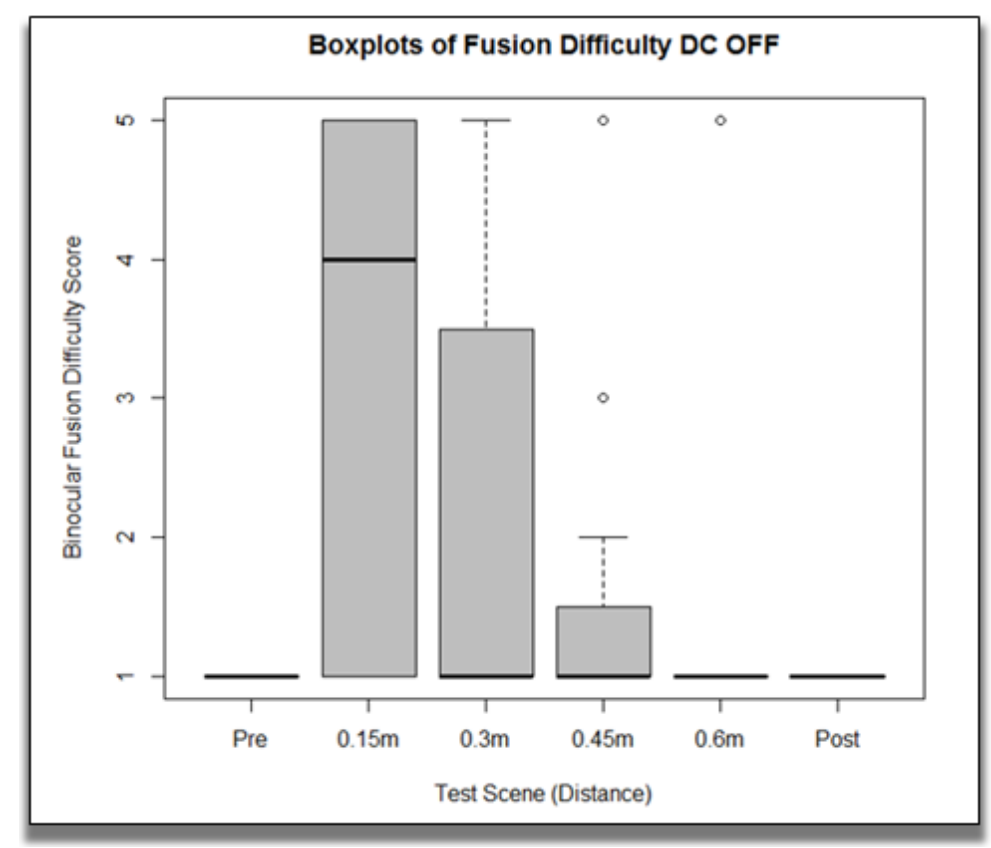

Figure 5.13: Shows box-plot of fusion difficulty scores with DC disabled.

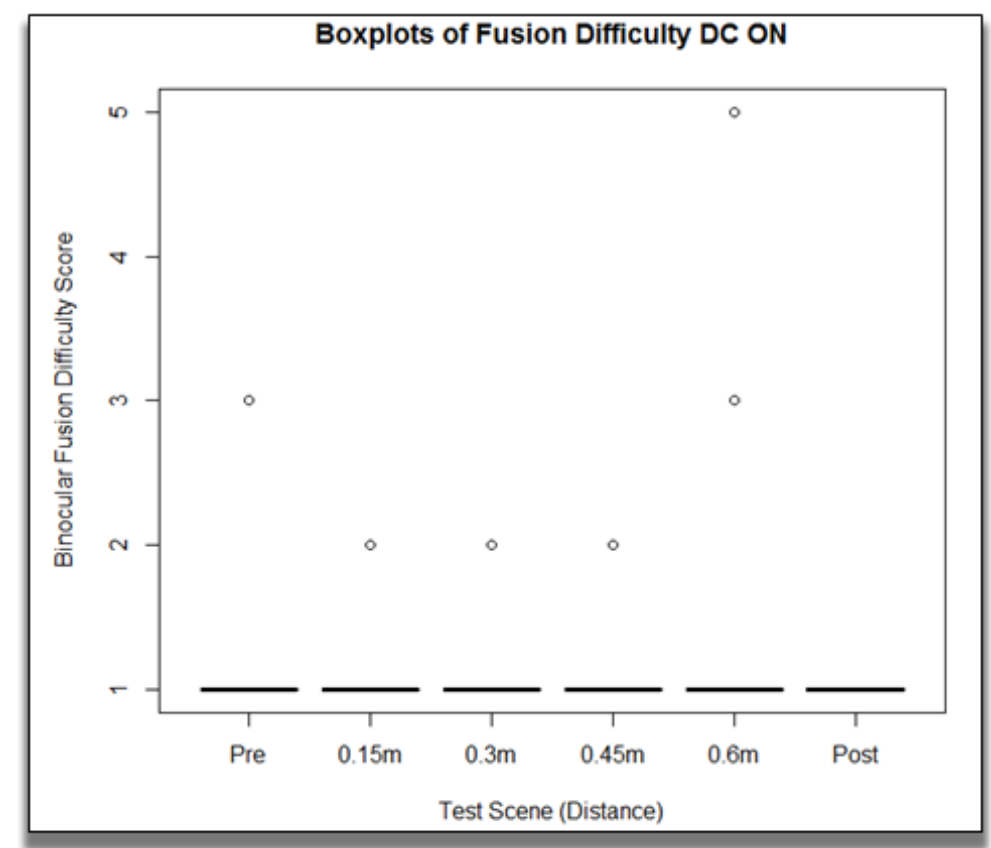

Figure 5.14: Shows box-plot of fusion difficulty scores with DC enabled. 
Figure 5.12 also suggests that, as found previously, many participants found it extremely difficult to view the stationary object at the shorter distances with DC disabled. This is also illustrated in Figures 5.13 and 5.14 Where the respective box-plots show the binocular fusion difficulty scores with DC disabled and enabled. In particular at the $0.15 \mathrm{~m}$ distance it can be seen that the median score reduces from 4 with DC disabled (Figure 5.13) to a value of 1 for all users with DC enabled (Figure 5.14), ignoring the outliers from the $1.5 \times$ Inter-Quartile Range (IQR) rule.

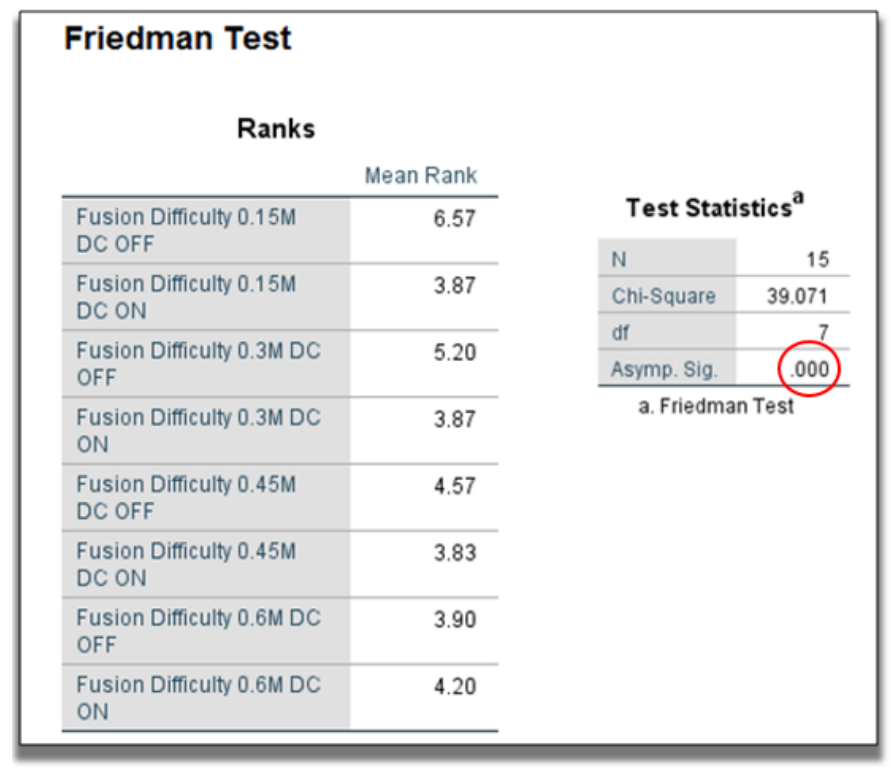

Figure 5.15: Results of the Friedman Test for DC ON / DC OFF

Repeating the statistical calculations as done previously, firstly running a Friedman test to compare the Mean Rank scores between DC enabled and DC disabled, Figure 5.15 shows the initial findings. It can be seen from the respective Mean Rank Scores that the greatest differences and hence therefore the values of most interest at this point are the first two closest distances $(0.15 \mathrm{~m}$ and $0.3 \mathrm{~m})$. Again, using the Wilcoxon Signed Rank Test to determine whether these differences were statistically significant. 
Reviewing the Wilcoxon Signed Rank Test results, it can be seen by Figure 5.16 that at the $0.15 \mathrm{~m}$ distance there are 10 Negative Ranks, which as explained in the key to the table in Figure 5.16 signifies that there are 10 occasions when participants scored ease of fusion difficulty lower (easier) with DC enabled than they did with it disabled at this distance. This means that two thirds of the participants noted an improvement with DC enabled. As the distance extended to $0.3 \mathrm{~m}$ there were just 6 participants that scored a lower ease of fusion difficulty with DC enabled (just over a third of the participants), 8 found no difference and one participant found the opposite. From a statistical significance point of view, as can be seen in Figure 5.17 both the shortest distances $(0.15 \mathrm{~m}$ and $0.3 \mathrm{~m})$ have a $\mathrm{p}$ value less than 0.05 . However, once the Bonferroni adjustment is applied (divide the original $\mathrm{p}$ value by the number of comparisons) the $\mathrm{p}$ value to determine statistical significance becomes $0.05 / 4=0.0125$. As can be seen from Figure 5.17 only the value for the $0.15 \mathrm{~m}$ viewing distance is less than this and therefore statistically significant.

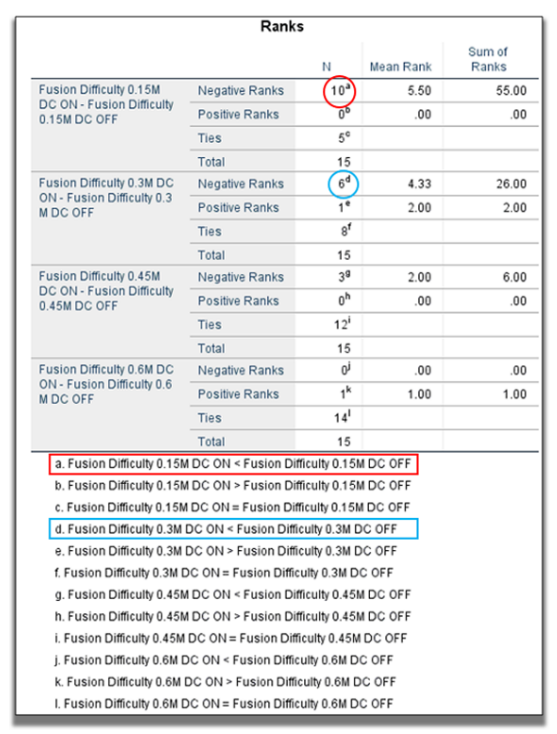

Figure 5.16: Results of the Wilcoxon Signed Rank Test for DC ON / DC OFF comparisons 


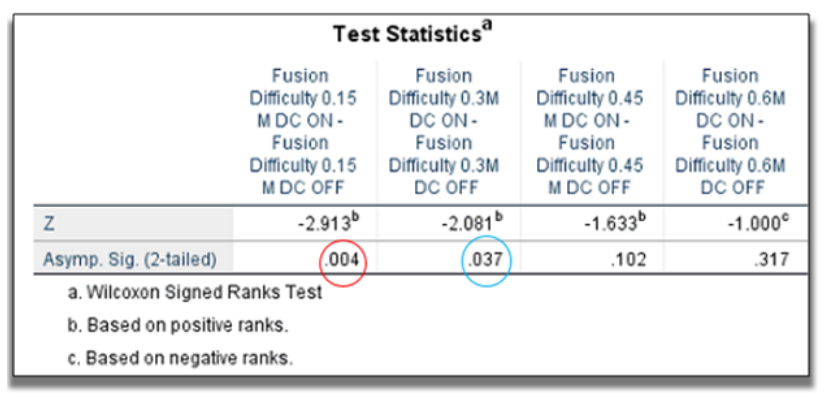

Figure 5.17: Shows the statistical significance results for the Wilcoxon Signed Rank Test for DC ON / DC OFF comparisons. As can be seen, there is significance at the distances of $0.15 \mathrm{~m}$ and $0.3 \mathrm{~m}$.

\section{Summary of Quantitative Statistical Analysis - For the Viewing of a Static Virtual Object}

The analysis in this section, with the use of the SPSS statistical software package has described and demonstrated the application of appropriate non-parametric statistical tests and techniques based on the specific sample survey data produced from the population in question. This has in turn produced defendable quantitative statistical inferences that suggest the following:

1. Confirmed that binocular fusion without DC enabled was more difficult when viewing a virtual stationary object at the shorter viewing distances.

2. Confirmed that with DC enabled, participants found it easier to perform binocular fusion when viewing a stationary virtual object at distances of $0.15 \mathrm{~m}$ and $0.3 \mathrm{~m}$. The former was shown to be statistically significant.

3. Confirmed that there were no other statistically significant discomfort symptoms evident when viewing a stationary virtual object across the four distances in question $(0.15 \mathrm{~m}-0.6 \mathrm{~m})$.

The results of this section of the analysis therefore suggest that 
with DC enabled participants benefited from being able to perform binocular fusion more easily.

\subsubsection{Analysis of Session 1 Part 2 - Instantaneous Move- ment Between Distances}

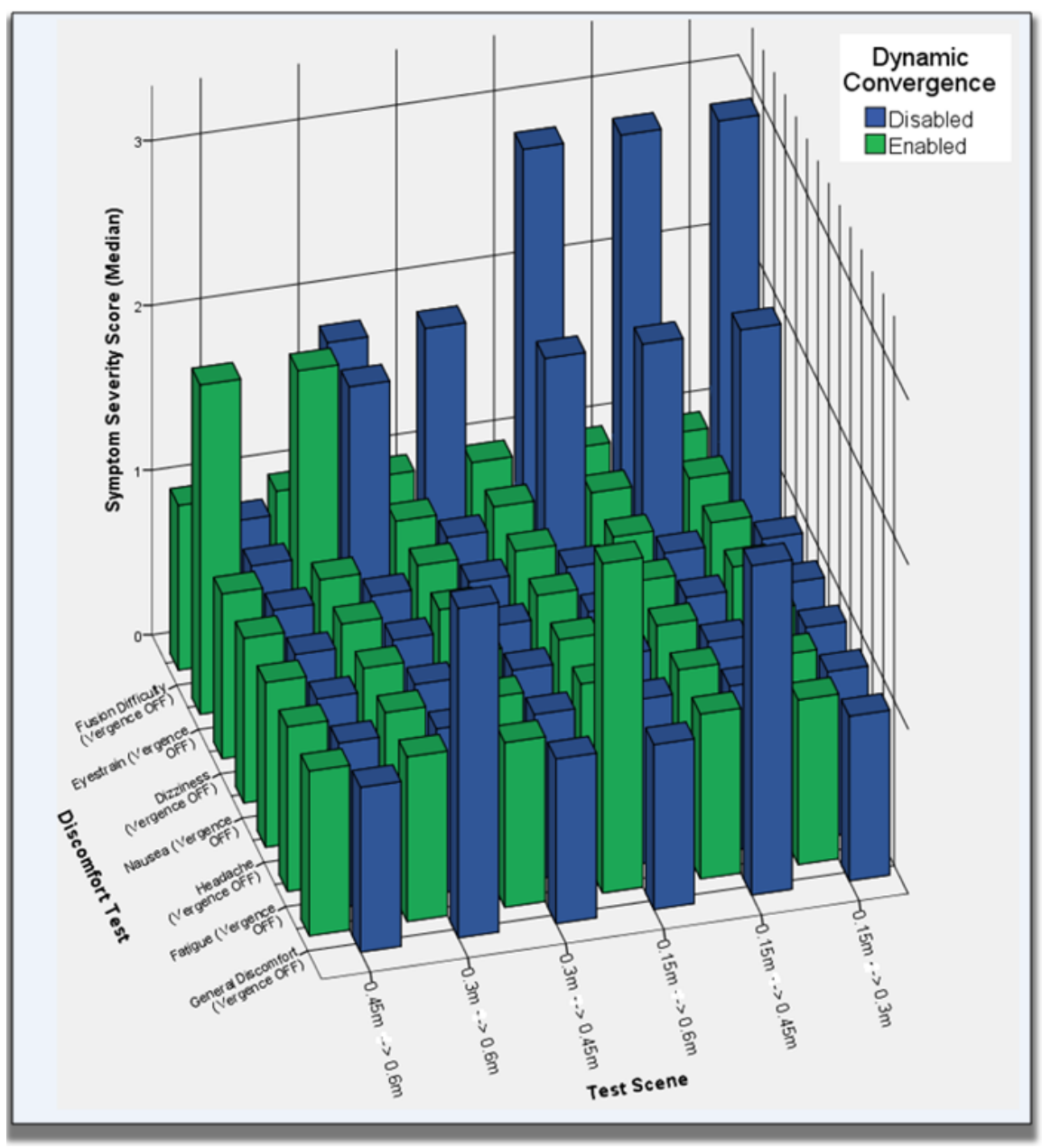

Figure 5.18: Shows the Median symptom severity scores for the periodic and instantaneous movement of a close virtual object

Following on from what has been found in the previous section, 
emphasis in this section of the analysis is on measuring the strength of evidence that supports the positive impact DC has with regard to the ease of fusion difficulty. However attention was also paid to reviewing any evidence for other discomfort symptoms. As described in Section 5.1.3, this part of the evaluation involved the participants looking at the periodic and instantaneous movement of a close virtual object between a pair of distances. A chart of the median severity scores for all tests are shown in Figure 5.18. As can be seen from the bar chart, it appears that many of the notably different median scores suggest the greatest difficultly is in binocular fusion with DC disabled at the shorter spectrum of the distance scale. Therefore to validate these observations, the same procedure was followed as in the previous section.

Firstly, the following null hypothesis is derived and tested by running a Friedman Test in SPSS:

$\mathrm{H} 0=$ There is no difference in the ease of fusion difficulty scores across all six instantaneous movement test scenes with DC Disabled.

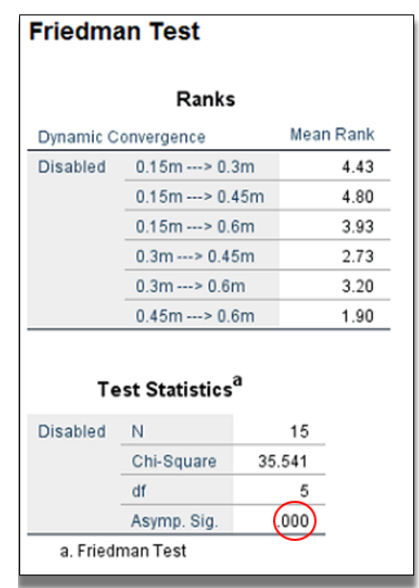

Figure 5.19: Friedman statistical test with DC disabled for instantaneous movement of close virtual objects indicated that there was a statistical significant difference in ease of fusion scores across the test scenes. 
As can be seen from Figure 5.19 the relevant statistical results are: $\left.\mathrm{x}^{2}(5, \mathrm{n}=15)=35.54, \mathrm{p}<0.05\right)$. It can be seen the critical chi-square value from the look up table (Figure 5.8 for $\mathrm{p}=0.05$ with $\mathrm{df}=5$ gives a value of 11.070). Therefore as indicated in 5.19 this initial result can be deemed as statistically significant and the above null hypothesis can be rejected, which means the following alternative hypothesis is accepted:

$\mathrm{H} 1=$ There are differences in the ease of fusion difficulty scores across all six instantaneous movement test scenes with DC Disabled.

Viewing the general trend of the Mean Rank scores in Figure 5.19, it can be seen that as the distance gets further away the ease of fusion score is lower, signifying that the closer distances become more difficult for viewing. This is evident from Figure 5.20 which shows how the median scores for the test scenes at the closest distance increase to 3 from the furthest test scene distances that have a median of 1 .

\begin{tabular}{|c|c|c|c|c|c|c|c|c|}
\hline \multicolumn{9}{|c|}{ Statistics } \\
\hline \multicolumn{3}{|c|}{ Dynamic Convergence } & $0.15 m \ldots 0.3 m$ & $0.15 \mathrm{~m} \rightarrow 0.45 \mathrm{~m}$ & $0.15 m \ldots 0.6 m$ & $0.3 \mathrm{~m} \ldots 0.45 \mathrm{~m}$ & $0.3 m \ldots 0.6 m$ & $0.45 m \ldots 0.6 m$ \\
\hline \multirow[t]{3}{*}{ Disabled } & \multirow[t]{2}{*}{$\mathrm{N}$} & Valid & 15 & 15 & 15 & 15 & 15 & 15 \\
\hline & & Missing & 0 & 0 & 0 & 0 & 0 & 0 \\
\hline & \multicolumn{2}{|c|}{ Median } & 3.00 & 3.00 & 3.00 & 2.00 & 2.00 & 1.00 \\
\hline
\end{tabular}

Figure 5.20: Shows median scores for instantaneous movement of close virtual objects test scene results with DC disabled.

Whilst the Friedman Test allowed us to rejection of the null hypothesis and accept the alternative (that there are differences in viewing distances with DC disabled), in order to find the statistical significance of the actual differences a Wilcoxon Signed Rank Test was run. The only significant results came from the three closest distances. As highlighted in Figure 5.21, it can be seen there are either 11 or 12 Negative Ranks (i.e. the number of occasions participants scored the closer distance scenarios with a higher value than the further distance and thus signifying a greater level 
of difficulty). It can also be seen from the figure, that the statistical significance is between 0.002 and 0.003 , which when applying the Bonferroni adjustment $(0.05 / 3=$ new $p$ Value is 0.017$)$ means that these are statistically significant and therefore this suggests that participants looking at the periodic and instantaneous movement of a close virtual objects with DC disabled find it more difficult at the closer distances.

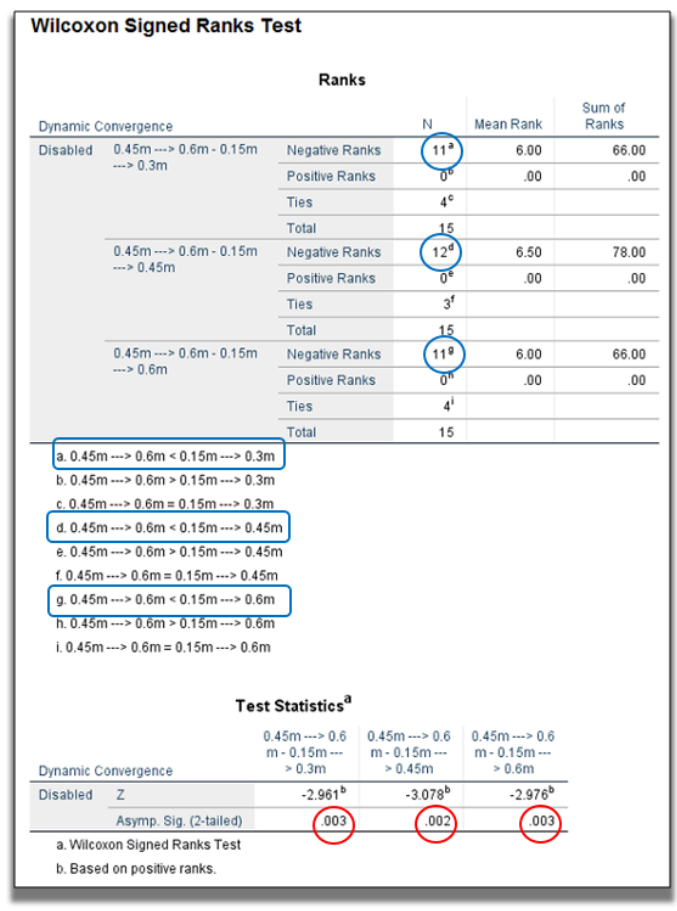

Figure 5.21: Wilcoxon Signed Rank Test for the most significant instantaneous movement of close virtual objects between DC enabled and disabled.

With confirmation that having DC disabled also has a detrimental effect on the instantaneous viewing of close objects, attention was then directed toward confirming how much of a positive difference could be attributed with having DC enabled for the instantaneous movement test scene scenarios. The Excel chart in Figure 5.22 shows binocular fusion viewing difficulty scores with DC ON/OFF for all participants for the in- 


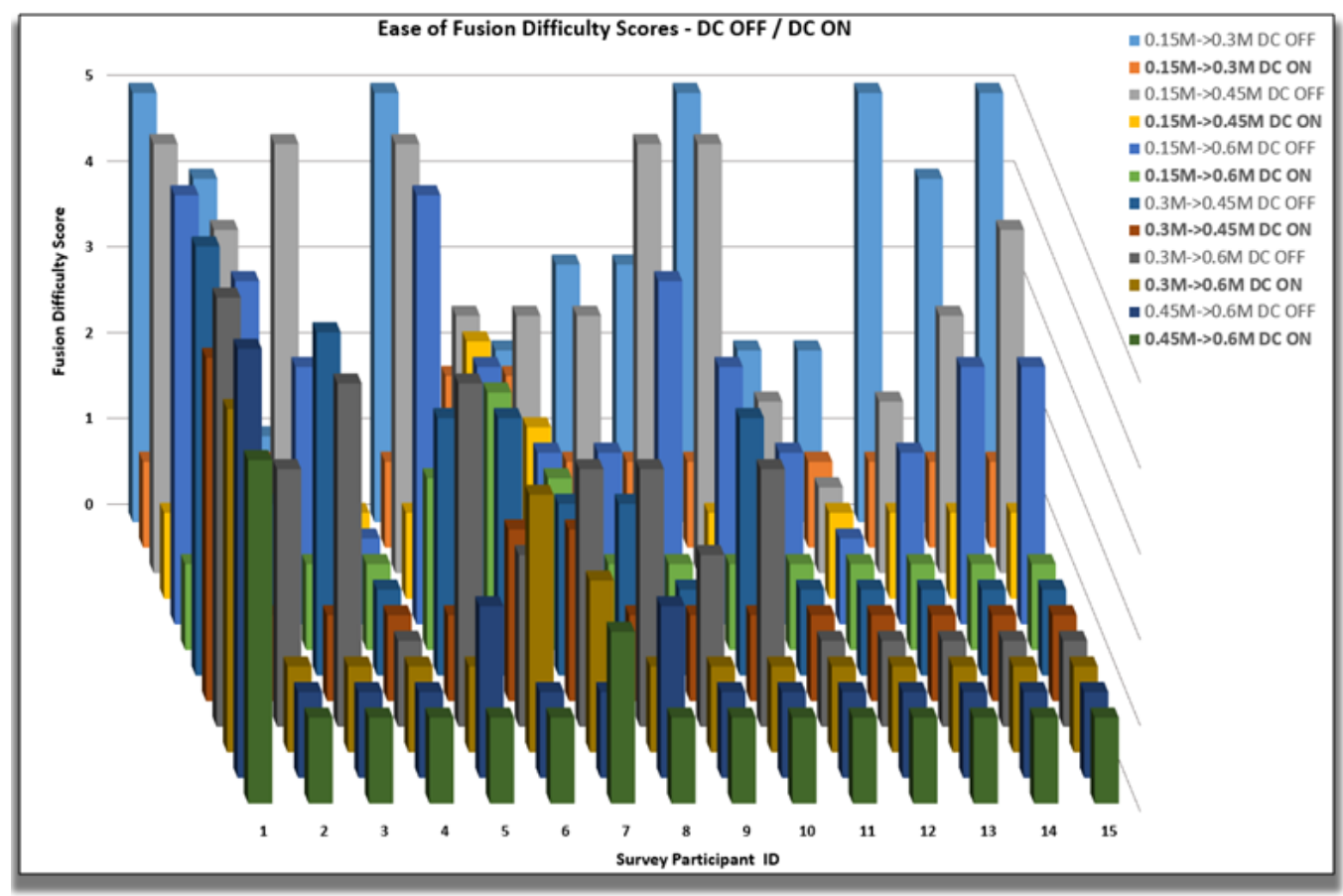

Figure 5.22: Shows binocular fusion viewing difficulty scores with DC ON/OFF for all participants for instantaneous transitions. As can be seen values are higher at closer distances when DC is off.

stantaneous transitions across six distances. As can be seen from the chart, it appears that the Fusion Difficulty score values are highest at the closest distances when DC is off.

As carried out previously a Friedman test was initially run as shown in Figure 5.23 to compare DC ON/OFF for fusion difficulty. The large differences between the Mean Ranks for DC OFF/ON are immediately evident at the respective transition distances. The given test statistic also indicates that there is a statistically significant difference in these sample population results. The critical chi-square value for 11 degrees of freedom is 19.67 to suggest a statistical significance. Clearly this is far surpassed with the value of 90.7 and a $p$ value of .000 . 


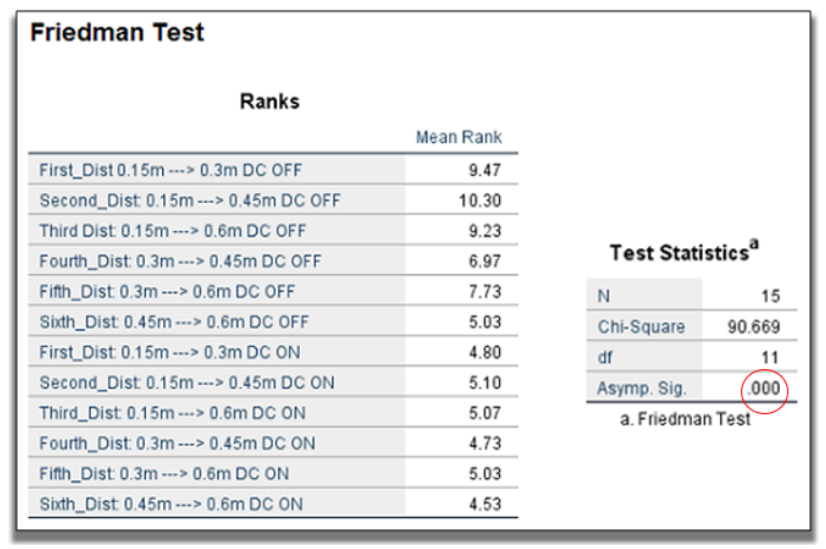

Figure 5.23: Shows Friedman result when comparing DC ON/OFF for fusion difficulty only. Note the differences between the Mean Ranks between $\mathrm{DC}$ OFF/ON at the respective transition distances. This also shows there is a statistically significant difference in these sample population results.

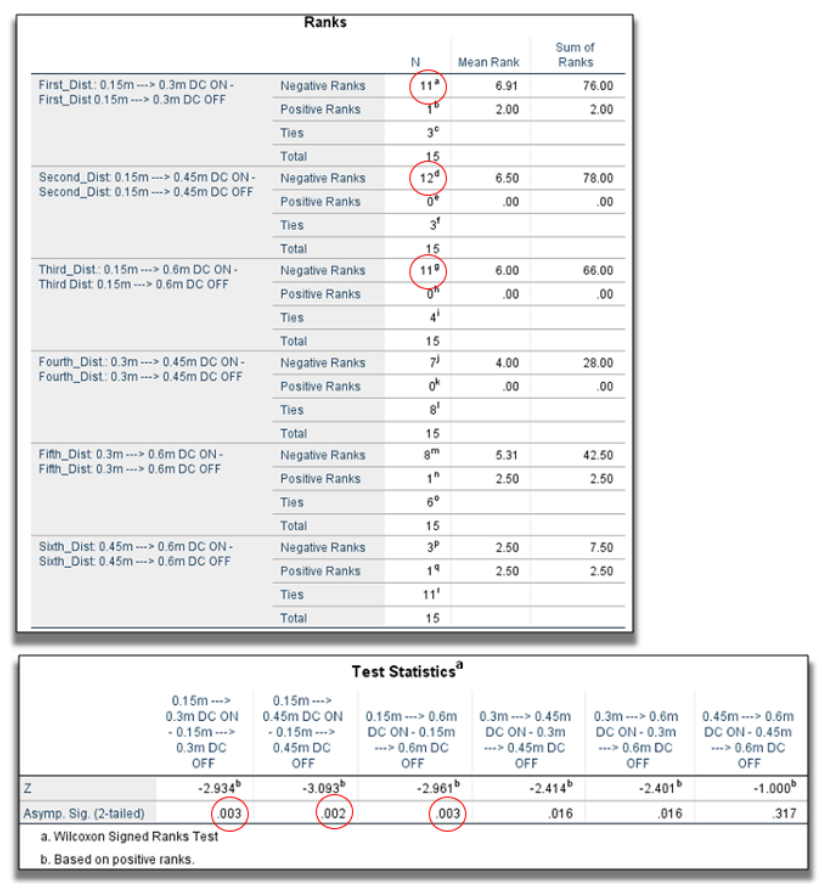

Figure 5.24: Wilcoxon Signed Rank Test for the most significant instantaneous movement of close virtual objects with DC disabled. 
Therefore to determine the actual statistical significance a Wilcoxon Signed Rank Test was also ran (Figure 5.24). While there were 5 total results below the p-value after applying the Bonferroni adjustment $(0.05 / 3$ = new $\mathrm{p}$ Value is 0.017), there were 3 that stood out the most at a statistical significance between 0.002 and 0.003 when compared to the other results of 0.016 . These 3 results are what will be focused on which are the three distances that contained $0.15 \mathrm{~m}$ as part of the transition $0.15 \mathrm{~m}$ $-->0.3 \mathrm{~m}, 0.45 \mathrm{~m}$ and $0.6 \mathrm{~m}$ ) with DC on and DC off. Figure 5.24 also highlights and similar to previous tests, that there are 11 or 12 Negative Ranks for those values of most interest (i.e. the first, second and third instantaneous object movements, which denote the number of occasions that participants scored that DC disabled with a higher value on the fusion difficulty score, signifying a greater level of difficulty). As in the previous similar test, the statistical significance results are again between 0.002 and 0.003 , which means that these are statistically significant. Therefore this suggests that participants looking at the periodic and instantaneous movement of a close virtual object with DC disabled will find it more difficult at the distances shown.

This finding is more easily observed from the simple box-plots. As can be seen from Figure 5.25 with DC off, the median value of fusion difficulty score is 3 at those first three distances that commence at $0.15 \mathrm{~m}$. The median value falls to 2 and then 1 at the furthest distance. Ignoring the outliers, it can be seen from Figure 5.26 that the median value is 1 for all distances with DC on. 


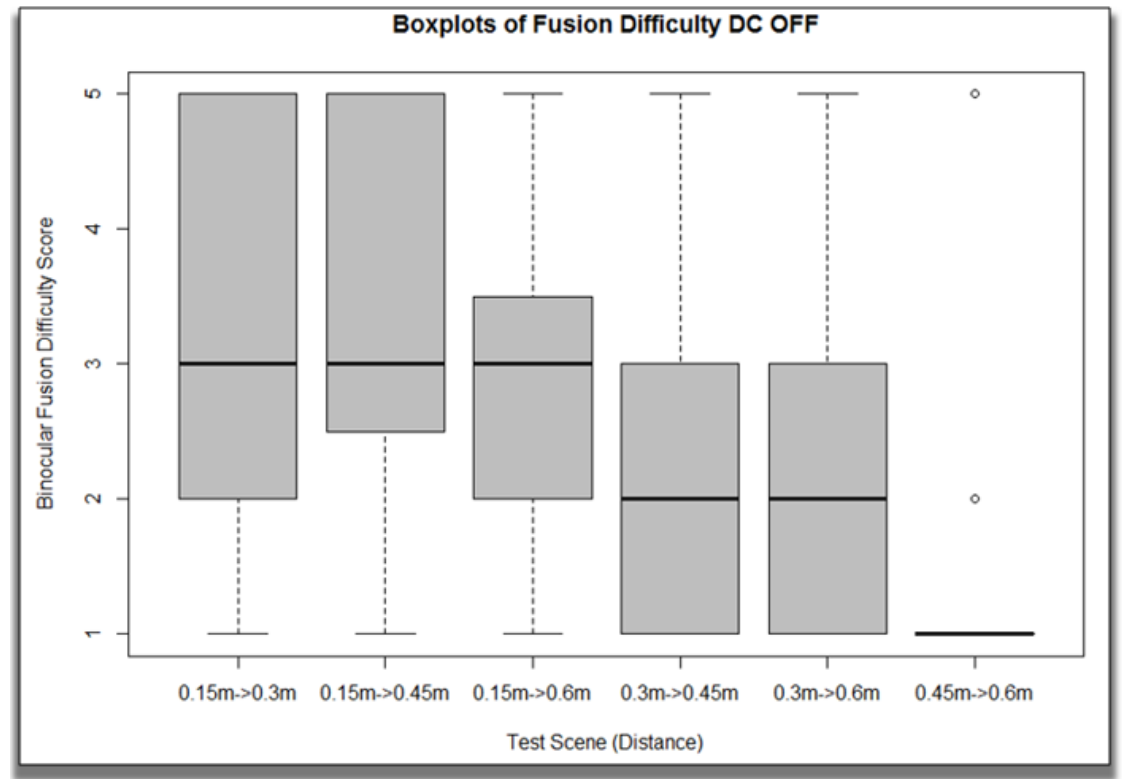

Figure 5.25: Shows the box-plot for binocular fusion viewing difficulty scores with DC disabled.

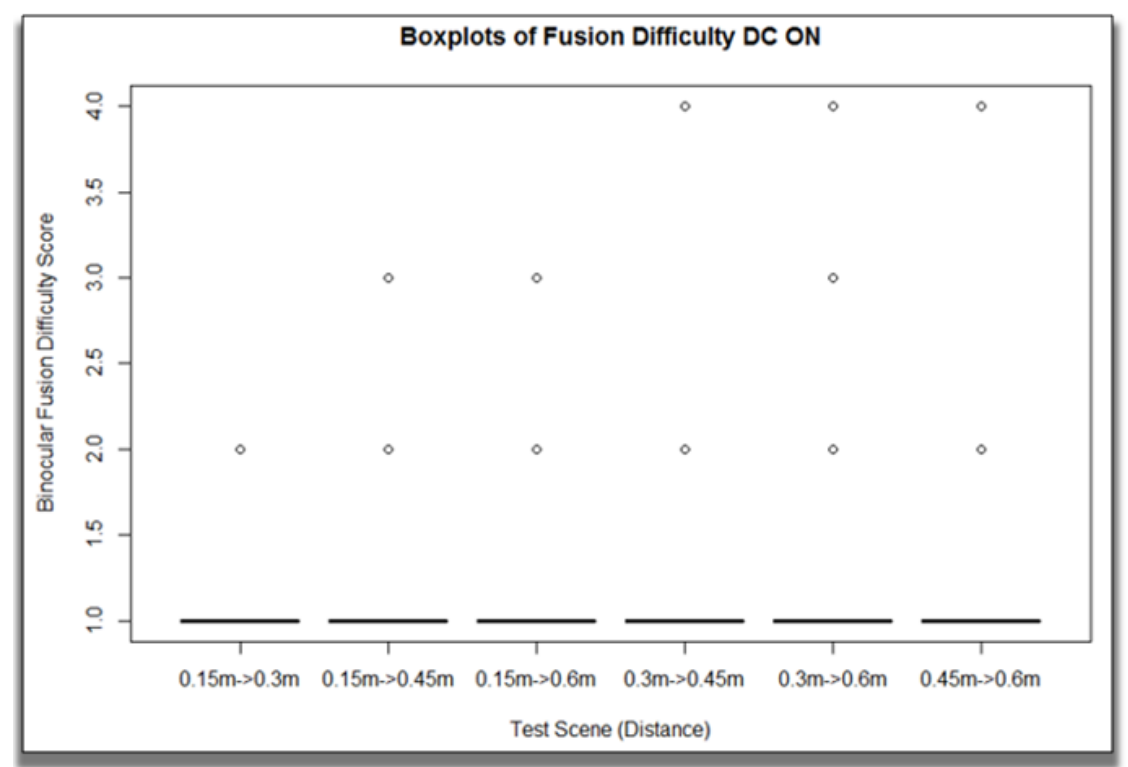

Figure 5.26: Shows the box-plot for binocular fusion viewing difficulty scores with DC enabled. 


\section{Summary of Quantitative Statistical Analysis - For the Instantaneous Movement of a Virtual Object}

With the use of the SPSS statistical software package defendable quantitative statistical techniques have demonstrably confirmed the following:

1. With DC disabled, participants found it more difficult to perform binocular fusion when viewing a virtual object that instantaneously moved to/from a distance of $0.15 \mathrm{~m}$.

2. With DC enabled, participants found it easier to perform binocular fusion when viewing a virtual object that instantaneously moved to/from a distance of $0.15 \mathrm{~m}$.

3. There were no other statistically significant discomfort symptoms evident when viewing the instantaneous movement of a virtual object across the six distances in question (between $0.15 \mathrm{~m}-0.6 \mathrm{~m}$ ).

The results of this section of the analysis therefore suggest that with DC enabled participants only benefited from being able to perform binocular fusion more easily for instantaneous movement of a virtual object, particularly at the closer distance of $0.15 \mathrm{~m}$.

\subsubsection{Analysis of Session 2 - Test Scenes with Interpolation}

Having determined that there is strong support that DC is effective for improving binocular fusion at closer distances, the status of DC is set to "ON" for all the remaining tests. The next set of analysis moves on to review the results of the experiments that looked at the impact of using interpolation as a transition from vergence depths while using DC. As described previously in Section 5.1.4, test scenes involved have two virtual 
objects at different vergence depths and participants were asked to move their head to look at them. All the test scenes and questions were repeated once for all of the three different types of interpolation (No, Linear and Ease Interpolation). This analysis covers both the quantitative responses from the likert scale and qualitative responses from the short interview.

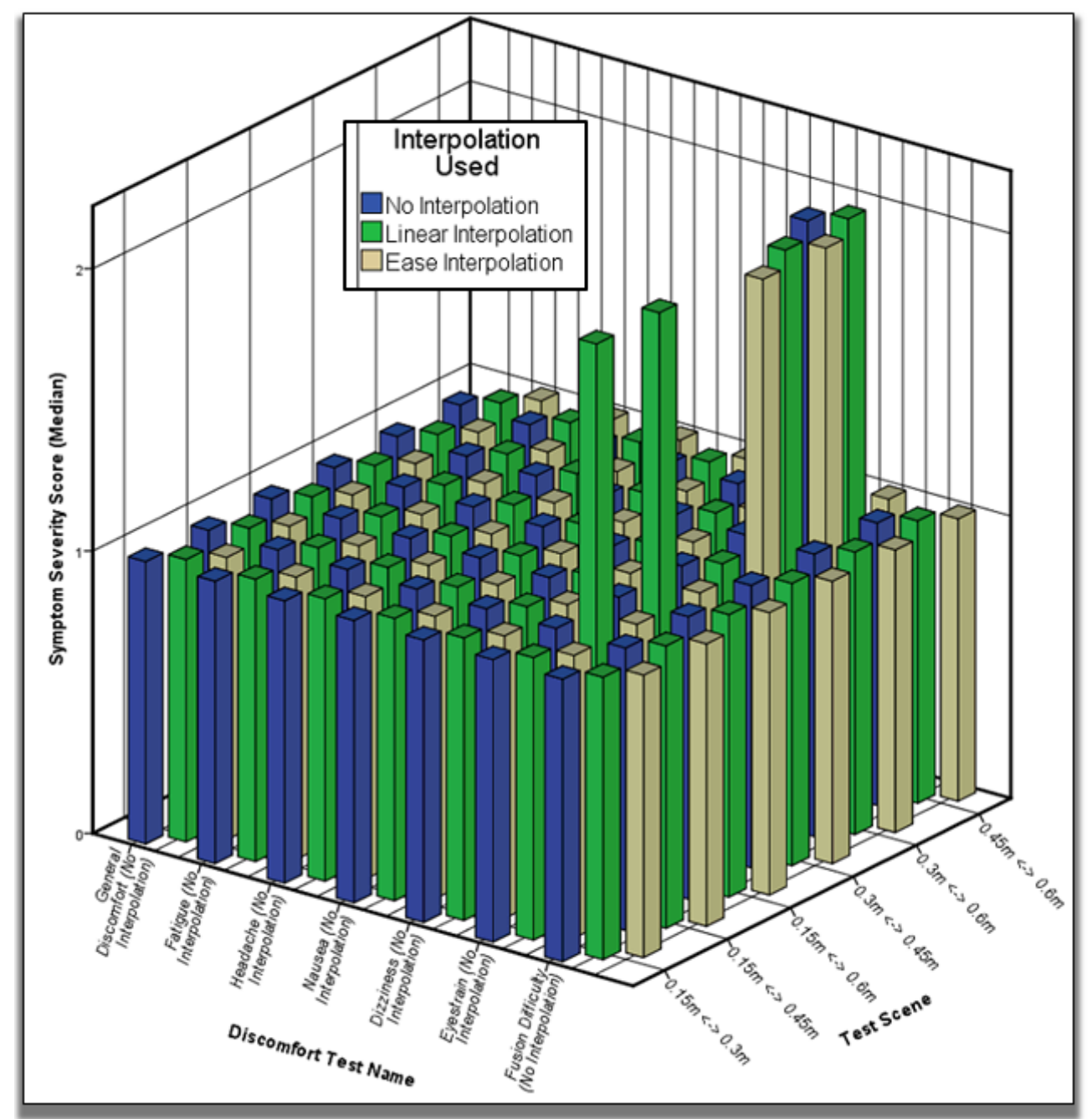

Figure 5.27: Shows the median discomfort scores across all test scenarios for all types of interpolation 
Figure 5.27 Shows the median symptom severity and difficulty scores across all test scene distances for all of the three interpolation scenarios. A Friedman test was ran to determine the amount of variance that existed within the respective scores. Firstly looking at how the three respective interpolation scenarios compared against each other and across the different test distances (See Figure 5.28).

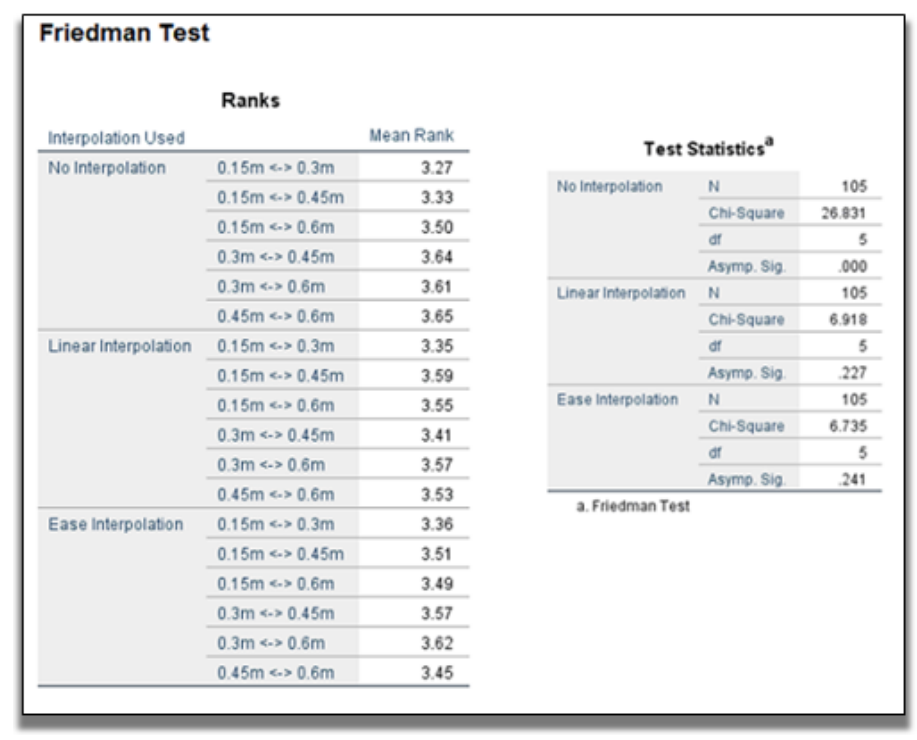

Figure 5.28: Results of the Friedman test

As can be seen by examining the Mean Rank scores in Figure 5.28 there is very little difference between them either between the respective interpolation options or between the various differences. Even though SPPS suggests that there may be some evidence to support statistical significance, numerous post hoc analysis tests through running many pairwise Wilcoxon Signed Rank tests found little to support this, therefore it was concluded that the differences are inconclusive.

However, as can be seen by the original chart of medians in 5.27 there does seem to be some evidence to suggest that there are some subtle differences with the reported scores related to the impact on eyestrain. 


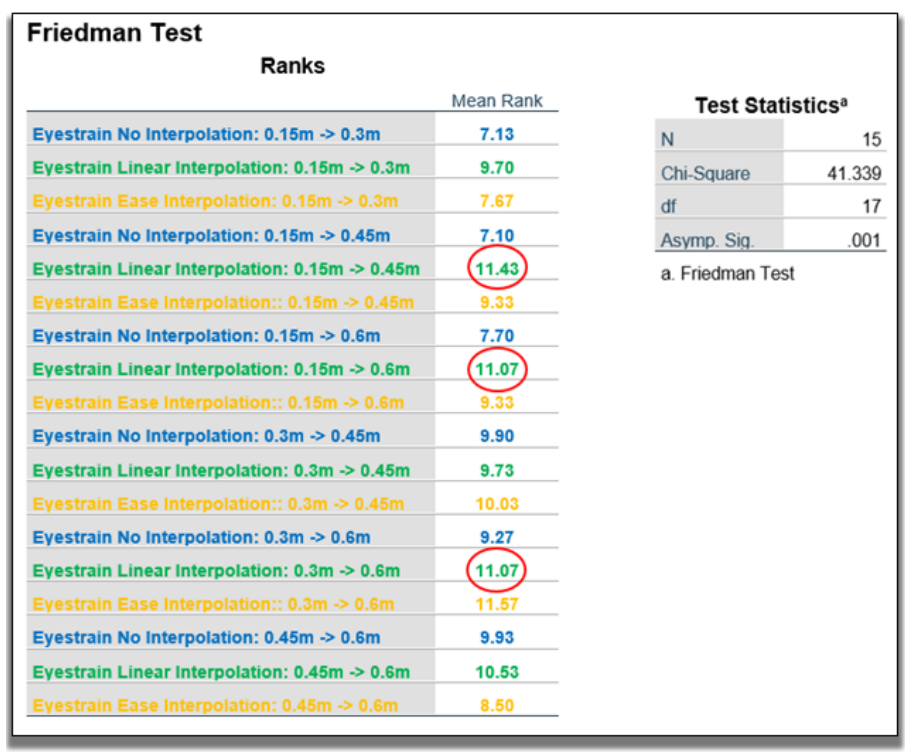

Figure 5.29: Results of the Friedman test for Eyestrain

Although this does not appear to be a large impact difference with having a Median increase from 1 to 2 . Nonetheless, another Friedman test was ran just for the eyestrain results to determine the level of statistical significance, as can be seen from Figure 5.29 there was some evidence of this being statistically significant since the test statistic provided a chi-square value of 41.3 and the $p$ value $=0.001$. Which is greater than the critical chi-square value of 27.59 from the distribution table at a significance level of 0.05 with 17 degrees of freedom (Number of tests - 1). As can be seen the text in Figure 5.29 has been highlighted to match the colours of the respective interpolation technique in the earlier chart (Figure 5.27) for ease of reading. It is evidenced from the Mean Rank scores that Linear Interpolation has slightly higher values than the other two interpolations. Three of the more notable instantaneous distances are pointed out in the figure denoting that there was some (albeit still slightly) greater discomfort for a number of participants, when using Linear interpolation however on running further post hoc pairwise Wilcoxon Signed Rank Tests these were deemed to not be statistically significant. 


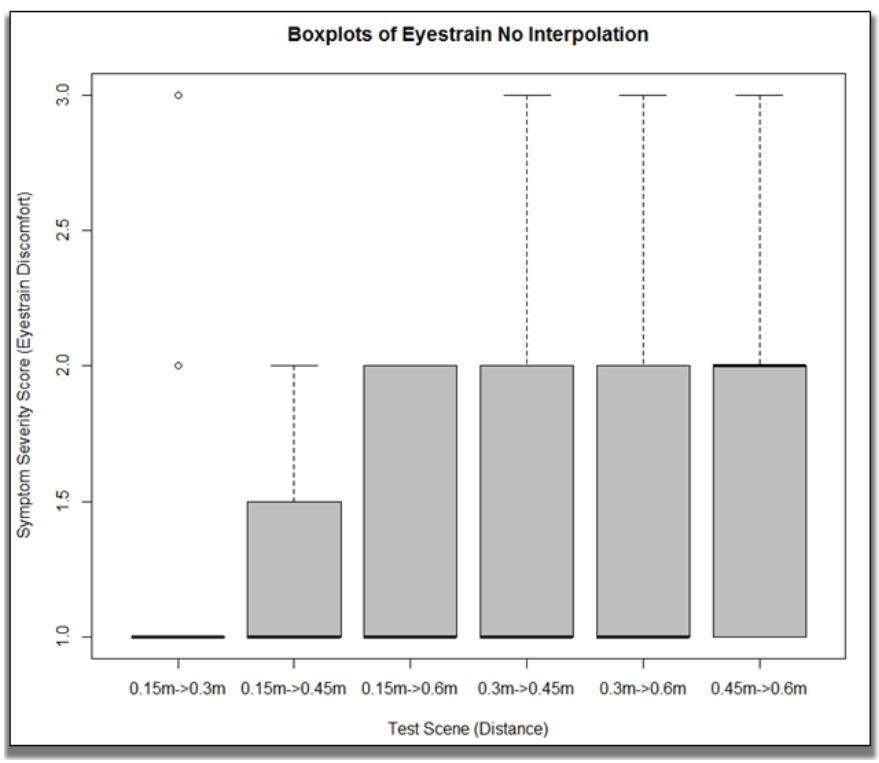

Figure 5.30: Show the box-plot for the severity of Eyestrain for No Interpolation.

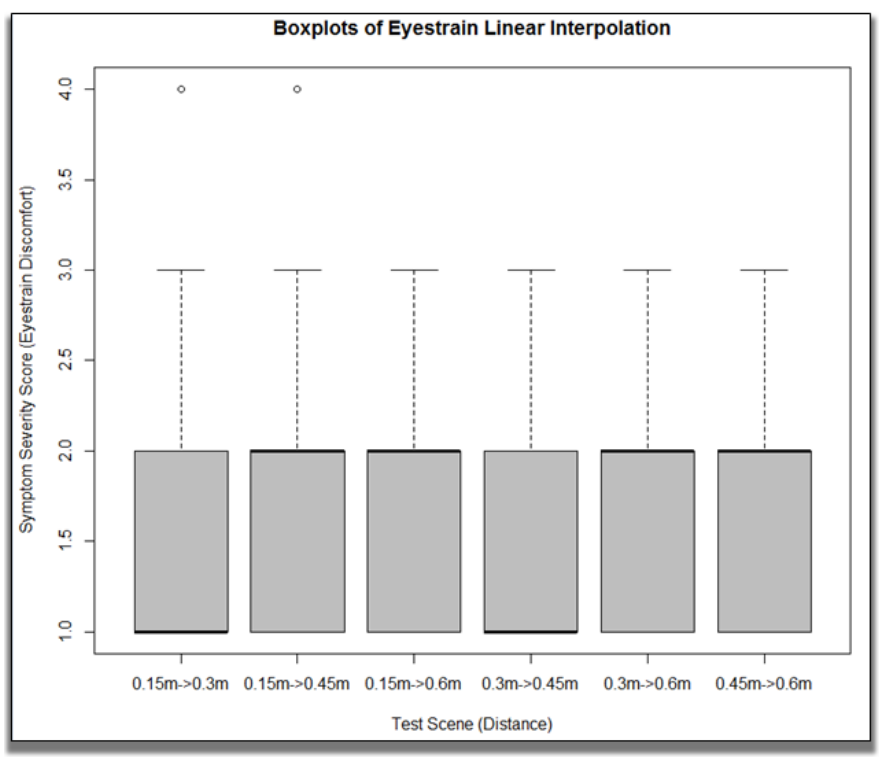

Figure 5.31: Shows the box-plot for the severity of Eyestrain for Linear Interpolation. 


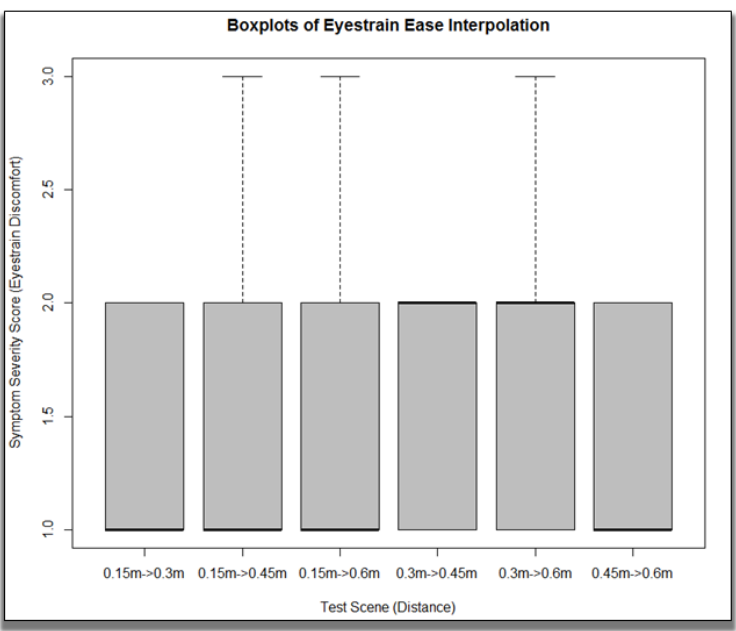

Figure 5.32: Shows the box-plot for the severity of Eyestrain for Ease Interpolation.

The relative difference between the scores and how they impact eyestrain can more easily be seen through examining the three box-plot diagrams in Figures 5.30, 5.31 and 5.32. Firstly, with 'No interpolation' (Figure 5.30) shows that the first distance (closest instantaneous transition between $0.15 \mathrm{~m}$ and $0.3 \mathrm{~m}$ ) scores is the lowest with the majority of participants scoring the value of 1 , with a couple of outliers scoring 2 and 3 respectively. The remaining distances for No interpolation show variability between 1 and 2 with a few scoring 3 . From this there is some small evidence that more participants found the shorter distance less discomforting with regard to eyestrain without interpolation.

The box-plot for 'Linear Interpolation' (Figure 5.31 shows that participants scored a mix of 1-2 across the various distances, with an occasional value of 3 and a couple of $4 \mathrm{~s}$ which can be seen as outliers. This suggests there may be some slight discomfort increase in eyestrain for some with linear interpolation. Finally, the box-plot for 'Ease Interpolation' Figure 5.32 shows a mix of variability across the distances which is inconclusive. 


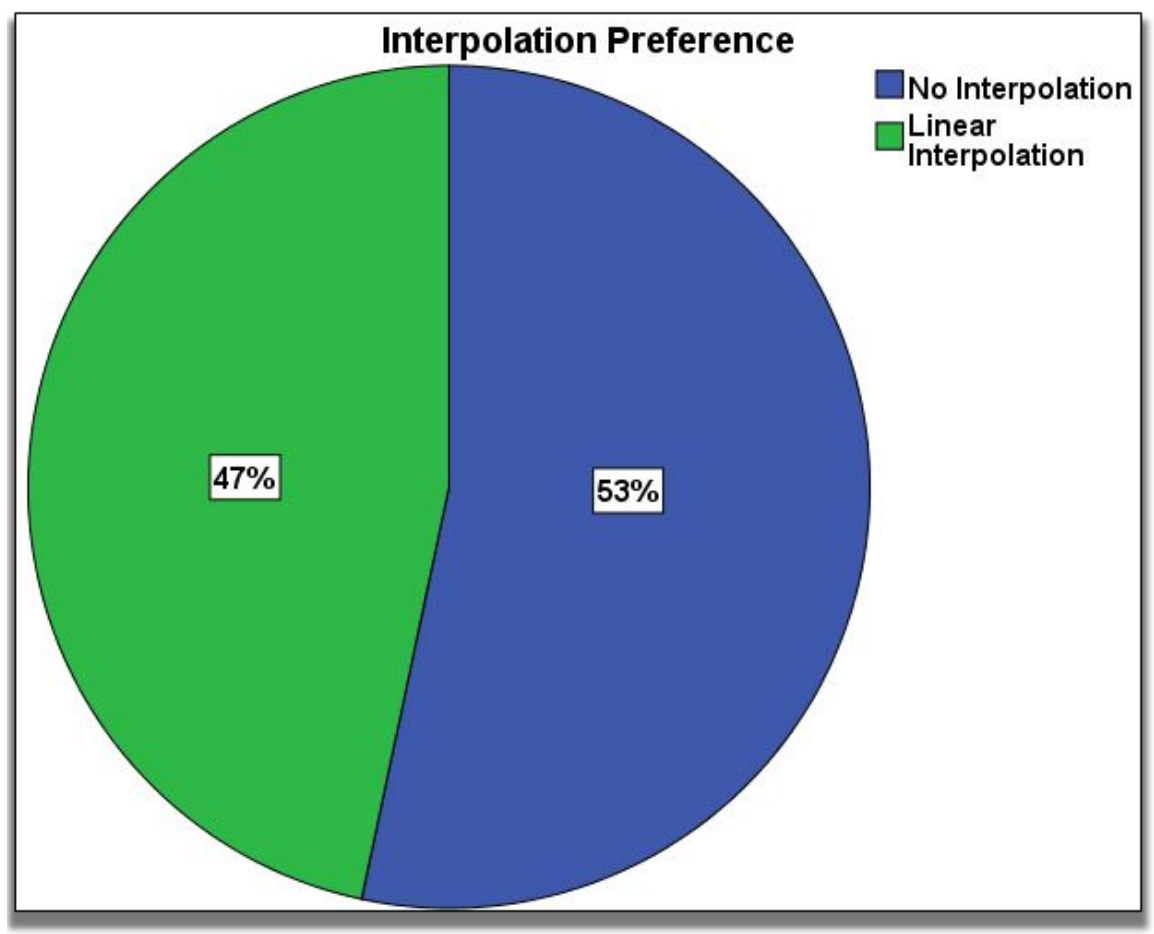

Figure 5.33: Shows the user preference percentage split for each mode of interpolation.

Looking at the qualitative part of the results, Figure 5.33 shows the chosen preference of interpolation from the short interview. It can be seen from this that there is a clear split in preference between 'No Interpolation' and 'Linear Interpolation', while 'Ease Interpolation' was not preferred by any participant.

From interviewing the participants, those who preferred having 'No Interpolation' commented on how it was "fast to merge" with "a clearer image". The "clearer image" comment refers to the fact that having no interpolation meant not having a brief moment of double vision during rotation of the virtual cameras. Those who did not prefer no interpolation commented on it being "too jerky" which caused some minor discomfort. 
When 'Linear Interpolation' was preferred, participants commented that "having a smoother transition was better" when compared to the "jerkiness" of having no interpolation. Those who disliked linear interpolation talked about how it was "slower to merge" and 'not as clear' which refers to the moments of some double vision being experienced.

The third choice, ease interpolation, was not preferred by any participant. The comments on ease interpolation from the short interview mentioned it was "a more rapid change" making the transition between vergence depths "more noticeable and therefore more disorientating and annoying" which made it "take too long to merge".

The only clear message among all of the participants was the dislike of ease interpolation. The use of the bezier curve for the ease interpolation made the transition more rapid and noticeable, which the participants did not like.

Some comments on linear and ease interpolation talk about how it felt "unnatural", which was odd considering the virtual camera rotation was based off the vergence rotation of the human eyes. However the short interview also revealed that a number of participants were not aware of some of the intricacies of the human visual system, such as when we experience a moment of double vision during vergence.

The preference split of the participants could mean that neither linear interpolation or no interpolation is the solution and that a hybrid solution would be ideal. Another interpretation could be that there is no overall ideal solution as each person may have different expectations for the vergence transition. Thus both types of interpolation would be viable to different users. 


\section{Summary of Analysis - Test Scenes with Interpolation}

With the use of SPSS software and numerous charts the results of the analysis for the experiments with interpolation are as follows:

1. There is no statistical significant evidence to illustrate any difference in discomfort or ease of binocular fusion across the three interpolation methods when viewing instantaneous movements of a virtual object across various distances.

2. There was some small observable evidence to suggest that of all the symptoms, eyestrain is potentially impacted by interpolation differences. In particular, the closest consensus for all participants was that with no interpolation at the shortest distance movement.

3. There were no other statistically significant discomfort symptoms evident when viewing the instantaneous movement of a virtual object across the six distances in question (between $0.15 \mathrm{~m}-0.6 \mathrm{~m}$ ).

The quantitative results of this section of the analysis therefore conclude that the experiments with interpolation are inconclusive and further work would need be done in this area.

From the answers and comments from the interview, it was learnt that:

1. 'Ease Interpolation' was a more rapid and noticeable transition and was thus not preferred by anyone.

2. There was a split in user preference between 'No Interpolation' and 'Linear Interpolation' due to participants either preferring a fast transition or a smoother transition.

The qualitative results of this section of the analysis conclude that 
there is an element of subjectivity to the most ideal transition between vergence depths. Further work would need be done to test and possibly improve these transitions.

\subsection{Discussion}

From the results of session 1 there was a clear benefit for the use of DC for performing binocular fusion when a small vergence depth of approximately $0.15 \mathrm{~m}$ is involved. In addition there was no significant evidence to suggest that DC had any negative effects on viewing discomfort.

For session 2, there were no clear differences in discomfort symptom severity for any of the different types of interpolation. The user preference interview showed an even split for having no interpolation or linear interpolation, but unanimously stated the dislike of ease interpolation. However the interview did highlight the merits of qualitative data, as the quantitative data collected did not hint towards the divide between two types of interpolation.

This brings up some of the limitations of the use of quantitative data. That is, due to the complexity of humans, it is difficult to rule out and control all the variables in an experiment. In addition people do not always behave and respond in the same ways due differing experiences and meanings. Quantitative data is unable to take these into consideration, as an example from this experiment the 'severity of a symptom' is very subjective, and will vary in meaning between participants.

Criticizing our experiment further, it was noted that some participants had trouble quantifying the severity of a symptom they were experiencing. One reason for this may be that participants may have never experienced a high severity of a symptom, thus making it hard to accu- 
rately place their current severity of it. If this is the case it could explain why there was little difference in symptom severity scores between DC being enabled and disabled throughout the experiment, as participants were being reserved with their answers, and kept their severity scores on the lower side of the scale. Following this, participants found it easier to quantify fusion difficulty as they had references for both sides of the scale. The minimum score of 1 meant that there was no issue in performing binocular fusion, a maximum score of 5 meant that it was impossible to perform binocular fusion.

Thus as an improvement for asking questions to participants, it would be better to give them a choice with clear definitions or points of reference, which leads to the use of more data without a metric, but rather a vote, such as the preference question from the short interview that was performed. While the use of voting may have a problem of producing results that may not be precise and hard to replicate, this research can gain greater understanding of the impact from DC has on humans from the opinions and experiences of the participants themselves.

For further investigation, we would also like to improve upon the transitions that were used in order to attempt to find a best fit solution. As previously mentioned, there was a split preference between which type of interpolation was preferred. The criticism of 'Linear Interpolation' was that it was 'too slow' in terms of being able to perform binocular fusion. In order to improve this transition, we could decrease the time the interpolation takes. In theory this should be a compromise between 'No Interpolation' and 'Linear Interpolation', meaning that binocular fusion should be able to be performed quicker while still having a transition that is not 'jerky'.

Looking back at the experiment from session 2, it should also be noted that while the time it took to perform binocular fusion was differ- 
ent between the 'No Interpolation' and 'Linear Interpolation', there was no difference in terms of fusion difficulty. Thus fusion time is something to consider separately from fusion difficulty and is worth investigating further. 
98CHAPTER 5. A QUANTITATIVE EXPERIMENT ON DYNAMIC CONVERGENCE 


\section{Chapter 6}

\section{Further Experimenting on Dynamic Convergence with Moving Objects}

The goal of this experiment is to further investigate the effect DC has on moving close virtual objects in terms of visual discomfort and fusion difficulty, but this time taking data in the form of a vote/preference rather than a metric. This test will look into instantaneous discrete movement of a virtual object at varying distances, and continuous movement of a virtual object at different speeds. In addition, this experiment will incorporate the suggested improvements to the 'Linear Interpolation' transition by shortening its duration.

However due to the nature of recording votes rather than a metric, we cannot perform the same statistical techniques or rigor that were seen in the previous chapter. Thus, for this experiment only basic descriptive statistics are used in the analysis of the results. 


\subsection{The User Test}

To start with, this experiment will test to see if there is a notable difference caused by changing the completion speed of the 'Linear Interpolation' transition. Participants will be presented with three different speeds and tested to see if they notice any difference. If a difference is noted, then the most preferred duration will be used for the rest of this experiment.

The main part of this experiment will then test three different settings to find which one is most preferred among participants:

1. No Dynamic Convergence

2. Dynamic Convergence with No Interpolation

3. Dynamic Convergence with Linear Interpolation

This will be done for both the discrete movement and continuous movement of a virtual object, which splits the experiment into two parts.

For the experiment the participants' preference in terms of what they think is the 'best' setting and the 'worst' setting (if any) for a particular category or symptom will be recorded. This was chosen based off the data from the interview of the previous user test, where we can get more detailed data from participants when they make relative comparisons between all the settings, rather than using a likert scale as previously discussed in Section 5.3.

One oversight from the previous user study was that tested the interpolation transition test involved neck movement. The use of neck movement causes a slightly different behaviour in human eye movement, which may have affected participant's opinion on the transitions. Thus for 
this experiment their will be no need for neck movement, as the object of interest will move by itself (much like session 1 part 1 of the previous user test) in order to have the wanted change in vergence depth.

\subsubsection{Setup}

For this evaluation, the same method of recruitment of participants from the previous evaluation were used, which were detailed in Section 5.1.1. The advertisement for this study was an edited version of the previously used advertisement (B.2). The same testing hardware from Section 5.1.1 was also used. For this study, there were a total of 10 participants with ages ranging from 19 to 37 years old, of which seven were male and three were female. All participants were current or prior students of Victoria University of Wellington.

\subsubsection{Procedure}

Much like the previous evaluation, the test scenes used in the evaluation were kept simple to help isolate any unforeseen variables. For this evaluation, the test scenes only contained a one virtual object with a word written on it for the participant to focus on. However the background was changed from the previous evaluation to be a basic 'room'. It was thought that this minor change would help increase with the participant's immersion, allowing for more reliable results.

For calibration, an approximation of $64 \mathrm{~mm}$ was used as the participant's IPD. A VR scene with DC enabled was shown with a virtual object at $0.15 \mathrm{~m}$ away from the virtual camera, the participant was then asked if they could see a single clear object. Adjustments in IPD were made until the participant could achieve this. 


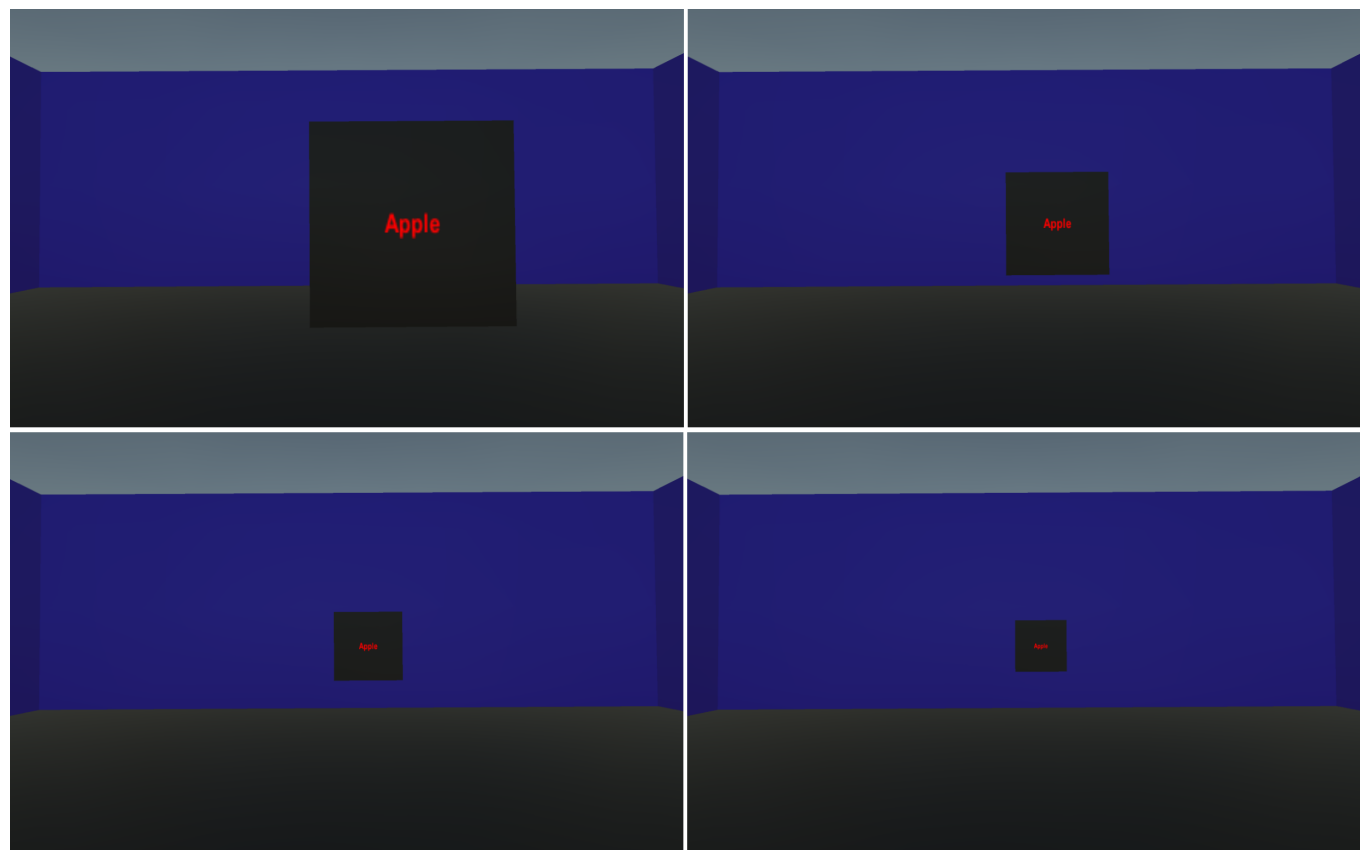

Figure 6.1: The distances used in the study, featuring the same virtual object at a vergence depth of $0.15 \mathrm{~m}, 0.30 \mathrm{~m}, 0.45 \mathrm{~m}$ and $0.60 \mathrm{~m}$ respectively.

\subsubsection{Transition Speed}

One of the previously mentioned methods of improving the linear interpolation transition was to decrease how long the transition takes, this is done by increasing the the speed of the angular rotation of the virtual cameras. From Section 3.4, it was explained that the original angular rotation speed used for the implementation was approximated to be about the same as the human visual system when it performs vergence ( 25 degrees/second).

For this part of the experiment, participants were shown a VR scene (Figure 6.1) with a virtual object periodically changing distances from $0.15 \mathrm{~m}$ to $0.6 \mathrm{~m}$ away from the virtual cameras, DC with linear interpolation was enabled. To investigate whether a faster transition was preferred, participants were given the option to freely change between three 
different "modes" which correspond with modifiers of 1, 2 and 4 to the rotation speed of the virtual cameras. The participant was then asked what mode they preferred (if any), the corresponding modifier was recorded and applied for the remainder of the evaluation. If there was no preference, the result was marked down as "N/A" and the original rotation speed was used for the evaluation.

\subsubsection{Questionnaire}

The evaluation was one session split into two parts to test discrete movement and continuous movement of the virtual object. Throughout the evaluation, participants were given the ability to switch between a number of 'modes'. Participants were asked to state what modes were the 'best' and 'worst' (if any) in terms of seven different categories:

- General Discomfort

- Eyestrain

- Nausea

- Dizziness

- Difficulty Fusing

- Fusion Time

- Overall

The questionnaire used for this evaluation can be found in Appendix B. 


\subsubsection{Discrete Movement}

For this part of the evaluation, participants were given the choice of three different modes label as "1", "2" and "3". These modes corresponded with the settings:

1. No DC

2. DC, No Interpolation

3. DC, Linear Interpolation

The set of questions were asked during each of six scenarios which were ordered randomly. Each scenario had a cube that would periodically change between two distances from the user. The scenarios used can be seen in Figure 6.2.

\begin{tabular}{|l|l|l|l|l|l|l|}
\hline Questions & $0.15-0.30$ & $0.15-0.45$ & $0.15-0.60$ & $0.30-0.45$ & $0.30-0.60$ & $0.45-0.60$ \\
\hline General Discomfort & & & & & & \\
\hline Eyestrain & & & & & & \\
\hline Nausea & & & & & & \\
\hline Dizziness & & & & & & \\
\hline Difficulty Fusing & & & & & & \\
\hline Fusion Time & & & & & & \\
\hline Overall & & & & & & \\
\hline
\end{tabular}

Figure 6.2: An excerpt for the discrete movement part of the questionnaire used in the second user evaluation (see Figure B.8 for the full questionnaire). The columns list the distance scenarios. The rows are the categories participants were asked to rank the three modes in terms of. 


\subsubsection{Continuous Movement}

For this part of the evaluation, participants were given the choice of two different modes label as "1", "2". These modes corresponded with the settings:

1. No DC

2. DC, No Interpolation

DC with 'Linear Interpolation' was omitted for this part of the experiment. This is because 'Linear Interpolation' would have no visual difference with 'No Interpolation' due to the continuous movement of the virtual object not having any big changes in the vergence depth.

Continuing, the set of questions were asked during each of two different scenes which were ordered randomly. Each scene had a cube that would periodically move between $0.15 \mathrm{~m}$ and $0.6 \mathrm{~m}$ from the user at a different speed. The different speeds were labeled as "Slow", "Medium" and "Fast" with modifiers of 1,2 and 4 respectively to the cube's speed. Figure 6.3 shows the table used for this part of the evaluation. 


\begin{tabular}{|l|l|l|l|}
\hline Questions & Slow & Medium & Fast \\
\hline General Discomfort & & & \\
\hline Eyestrain & & & \\
\hline Nausea & & & \\
\hline Dizziness & & & \\
\hline Difficulty Fusing & & & \\
\hline Fusion Time & & & \\
\hline Overall & & & \\
\hline
\end{tabular}

Figure 6.3: An excerpt for the continuous movement part of the questionnaire used in the second user evaluation (see Figure B.8 for the full questionnaire). The columns list the speed scenarios. The rows are the categories participants were asked to rank the two modes in terms of.

\subsection{Analysis 2}

In this section, the results of the second user evaluation are introduced and analysed. As noted earlier in this chapter, due to the recording votes rather than a metric, we cannot perform the same statistical techniques or rigor that were seen in the previous analysis. Thus, this analysis will only be using basic descriptive statistics to go over the results. Afterwards, the overall conclusions for this experiment are presented and compared to the results from the first user test.

\subsubsection{Transition Speed}

Figure 6.4 shows the preference of interpolation speed. $40 \%$ of the participants didn't notice any difference between the three speeds of interpolation. Of the participants who did notice a difference and had a prefer- 
ence, there was a mixed response in terms of which speed modifier was preferred. From these results a conclusion cannot be drawn on whether a faster or slower 'Linear Interpolation' transition is preferred, or if the speed of the transition has an impact at all.

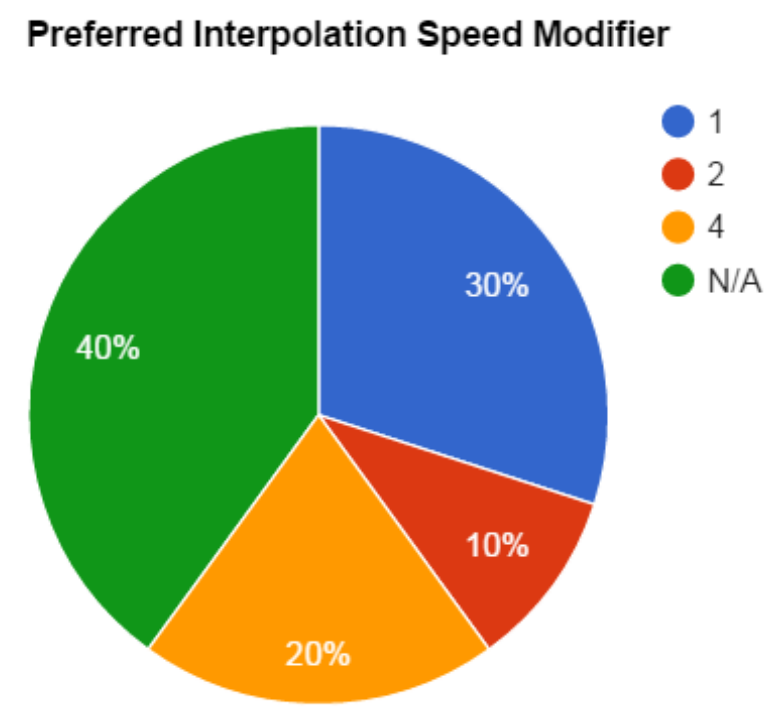

Figure 6.4: The percentage split of participants that preferred a particular linear interpolation speed.

\subsubsection{Discrete Movement}

Figures 6.5 through 6.18 illustrate the voted 'best' and 'worst' modes in terms of different categories, when looking at a close virtual object that moves instantaneously. For reference, the notations on the graphs mean:

- 1 - No DC.

- 2 - DC with No Interpolation.

- 3 - DC with Linear Interpolation. 
- N/A - No difference between the modes.

- $\mathrm{X}, \mathrm{Y}$ - No difference between mode $\mathrm{X}$ and mode $\mathrm{Y}$.

Looking at the categories General Discomfort (Figures 6.5 - 6.6) and Eyestrain (Figures 6.7 - 6.8), Mode 1 was very often voted to be the worst mode if any difference was noted. Meanwhile, Mode 2 was often voted the best mode or tied with Mode 3. Mode 2 was never considered to give the worst amount of general discomfort or eyestrain. Mode 3 had mixed opinions by being voted the worst, best or tied for best.

For both categories Nausea (Figures 6.9 - 6.10) and Dizziness (Figures 6.11 - 6.12), a good majority of participants (50\% to $70 \%$ ) did not notice any difference or experience any symptoms throughout the six different scenarios. Mode 2 was never considered to give the worst amount nausea or dizziness and was often considered to be the best mode. Other participants reported experiencing no difference in nausea or dizziness between Mode 2 and Mode 3, while those that did experience some nausea or dizziness felt that it was because of either Mode 1 or Mode 3.

The general consensus for Fusion Difficulty (Figures 6.13 - 6.14), Fusion Time (Figures 6.15 - 6.16) and the Overall preferred mode (Figures $6.17-6.18$ ) was that Mode 1 was the worst and Mode 2 was the best. Mode 3 had mixed opinions about it. Due to the similarity in results between these three categories, it can be assumed that Fusion Difficulty and Fusion Time had the biggest impact on the participants' decision of the overall preferred mode.

The only distance scenario that had stood out was $0.45 \mathrm{~m}-0.6 \mathrm{~m}$. For this scenario, general discomfort, fusion difficulty and fusion time all had mixed results in terms of the best mode when compared to the other distance scenarios of the same category. This scenario also had the most number of votes for noticing no difference among all the categories. 


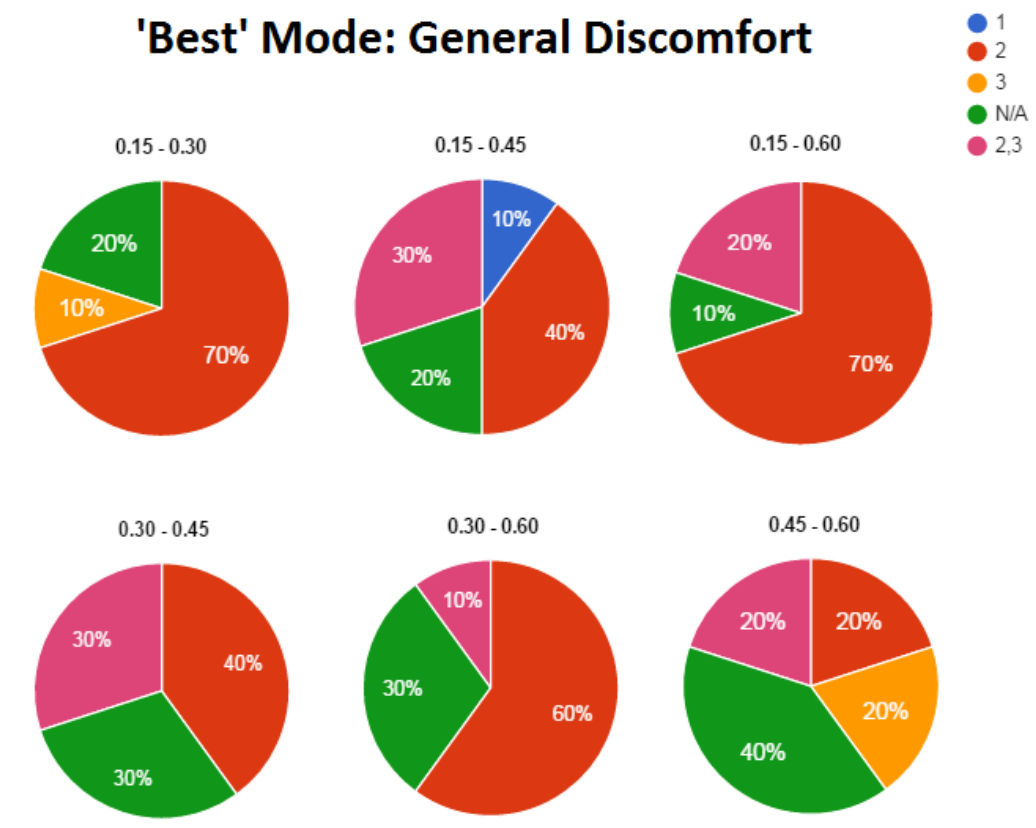

Figure 6.5: The number of participants that thought which mode(s) gave the least amount of general discomfort.

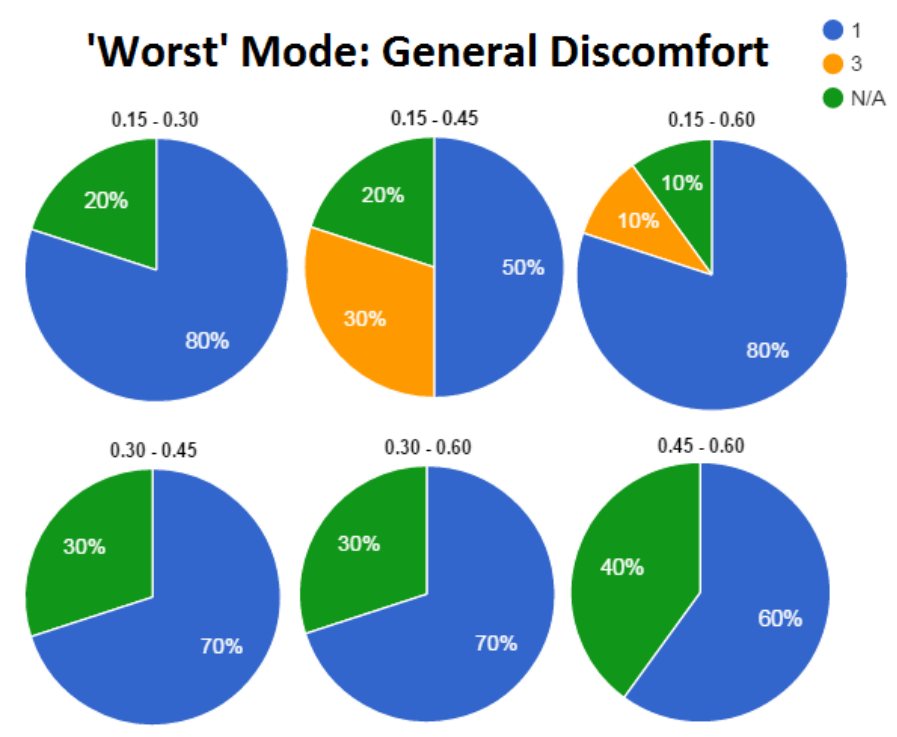

Figure 6.6: The number of participants that thought which mode(s) gave the most amount of general discomfort. 


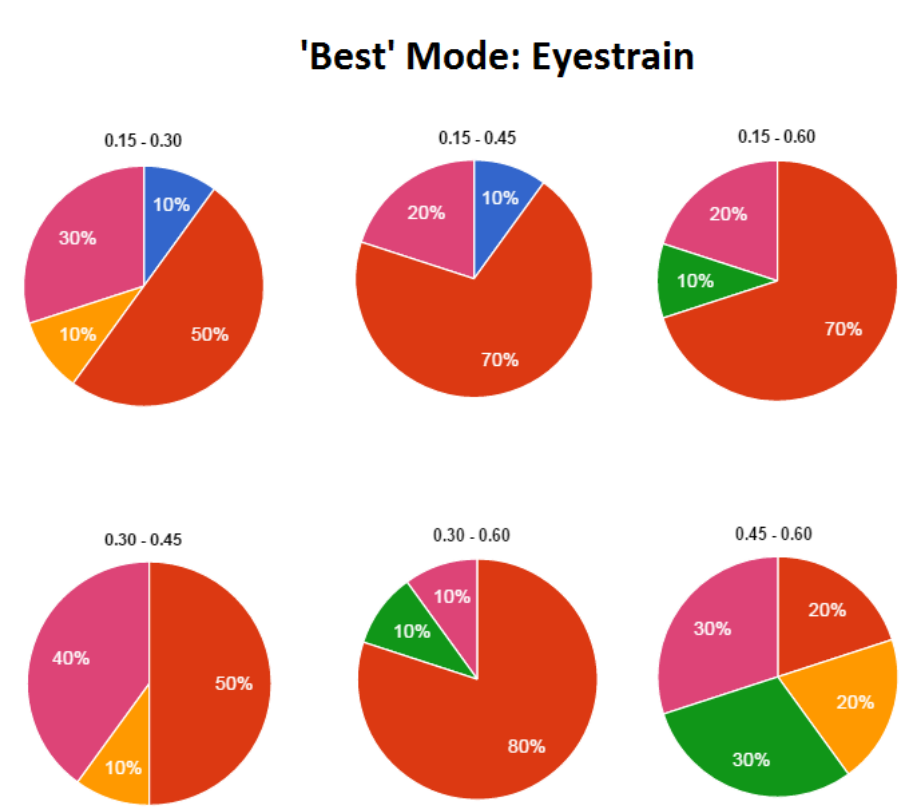

Figure 6.7: The number of participants that thought which mode(s) gave the least amount of eyestrain.

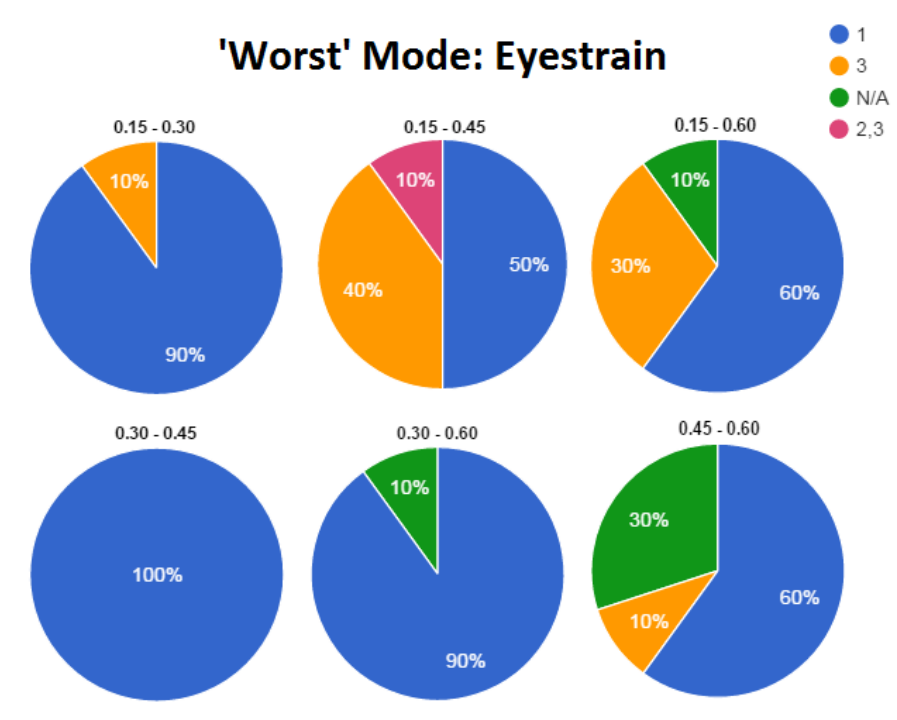

Figure 6.8: The number of participants that thought which mode(s) gave the most amount of eyestrain. 

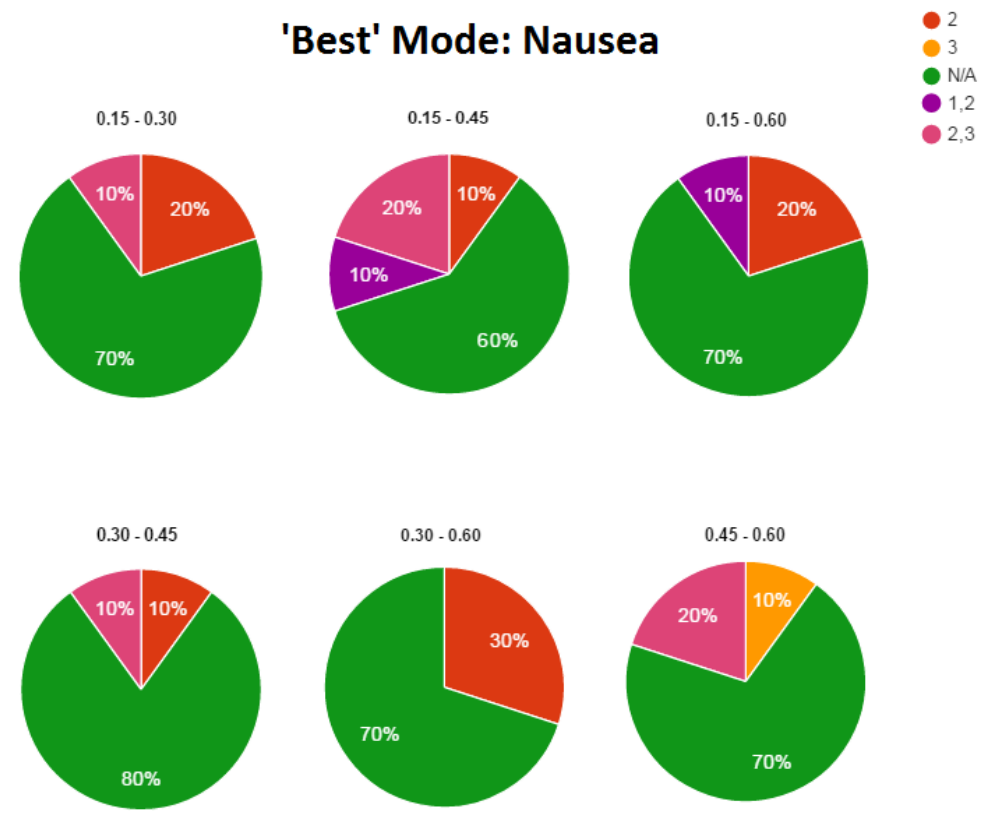

Figure 6.9: The number of participants that thought which mode(s) gave the least amount of nausea.

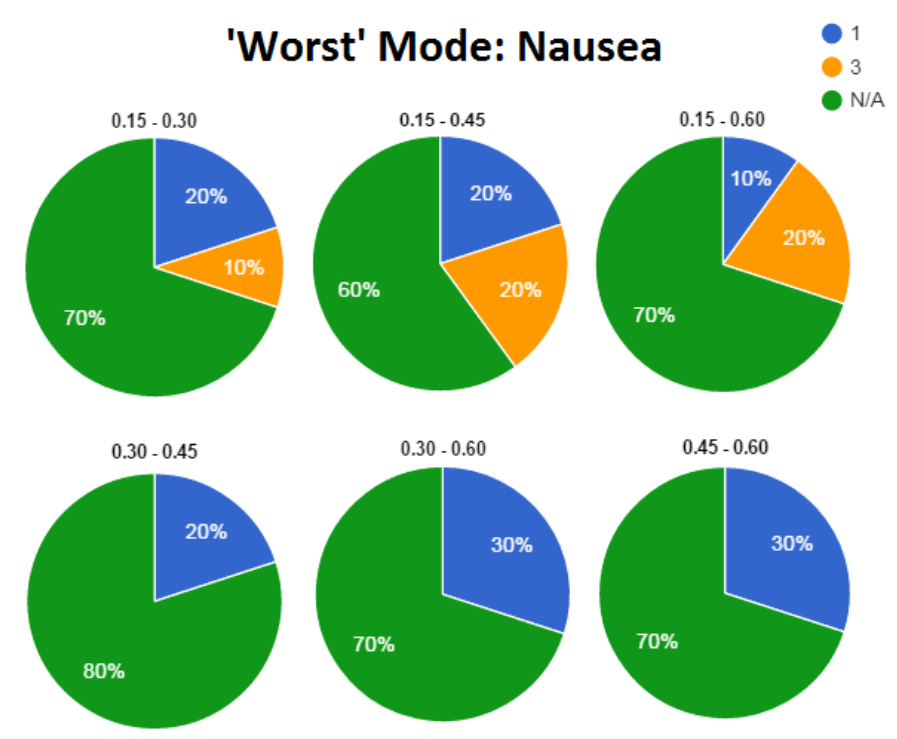

Figure 6.10: The number of participants that thought which mode(s) gave the most amount of nausea. 


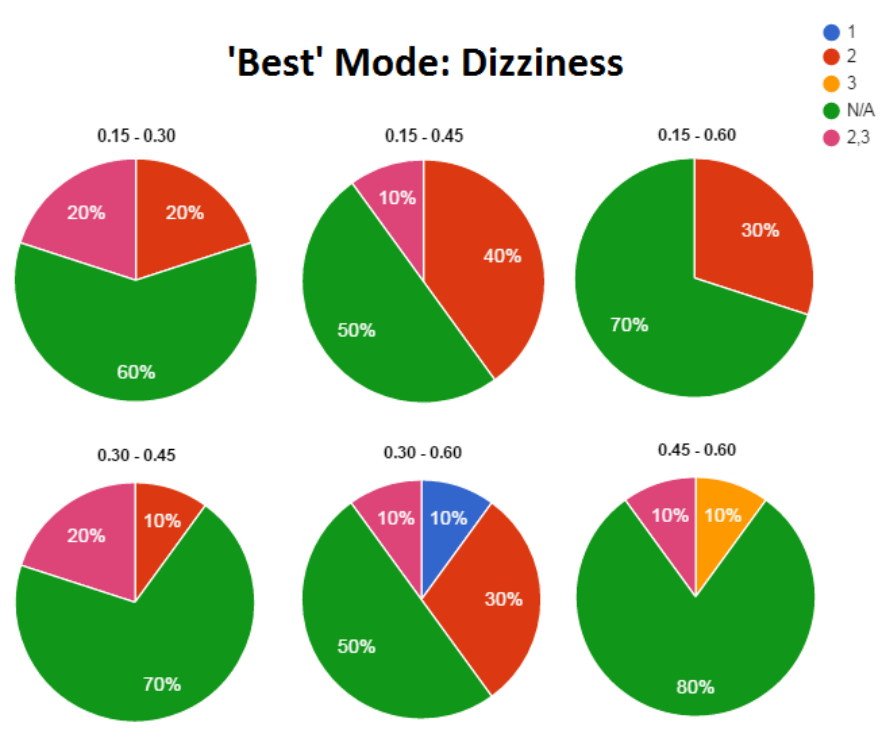

Figure 6.11: The number of participants that thought which mode(s) gave the least amount of dizziness.

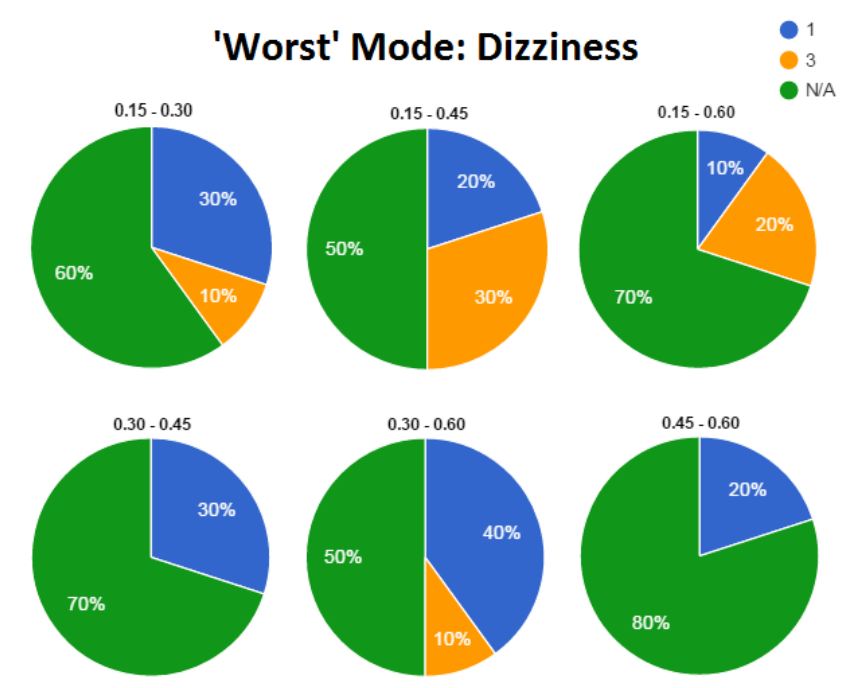

Figure 6.12: The number of participants that thought which mode(s) gave the most amount of dizziness. 


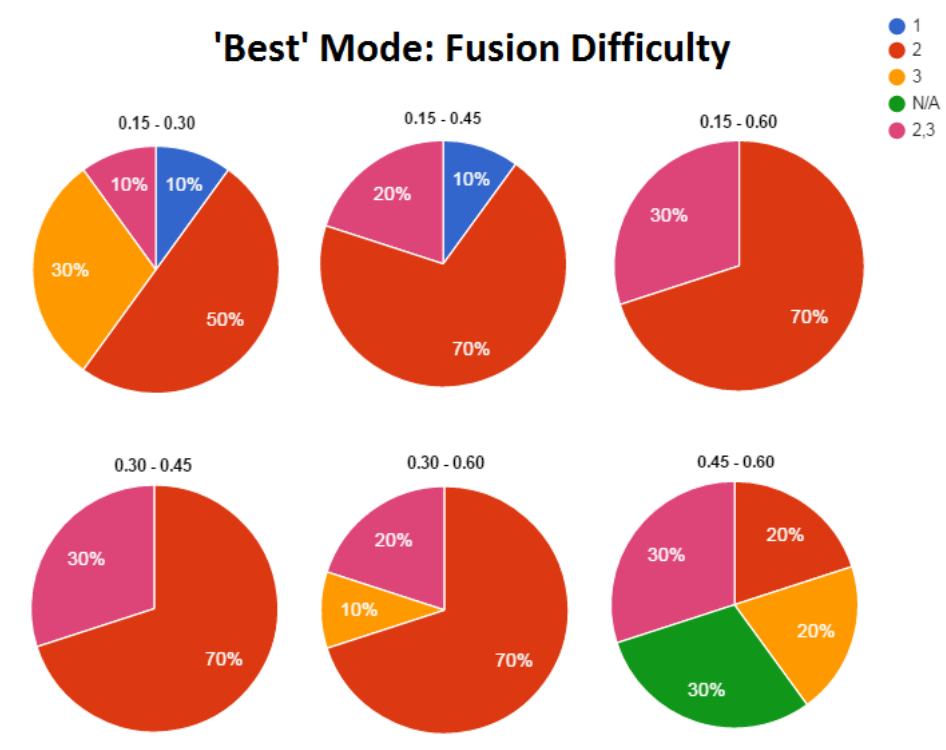

Figure 6.13: The number of participants that thought which mode(s) was the easiest to fuse with.

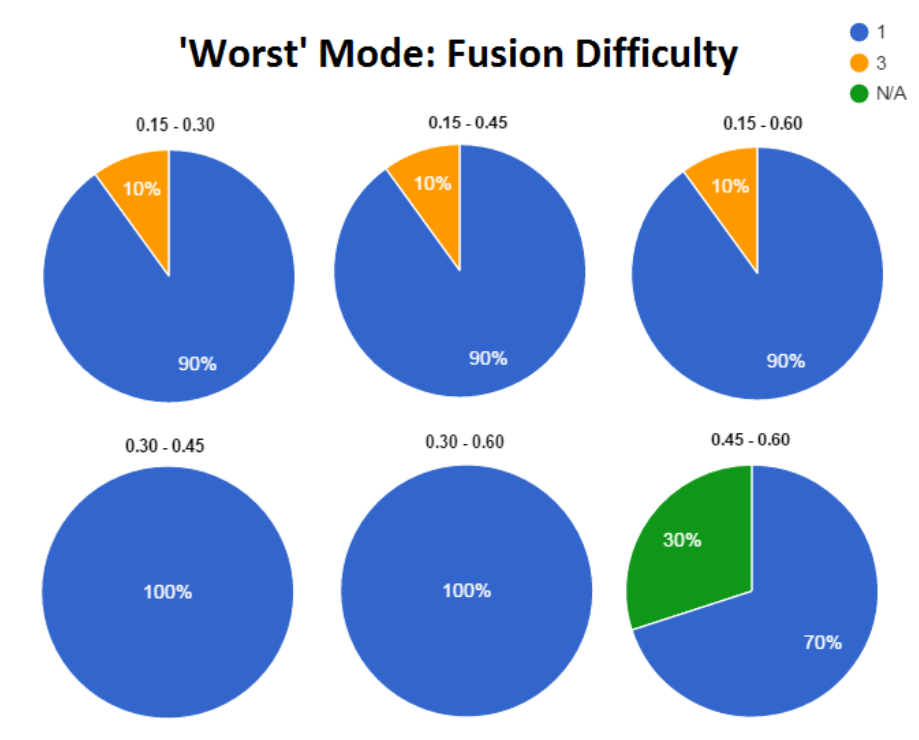

Figure 6.14: The number of participants that thought which mode(s) was the hardest to fuse with. 


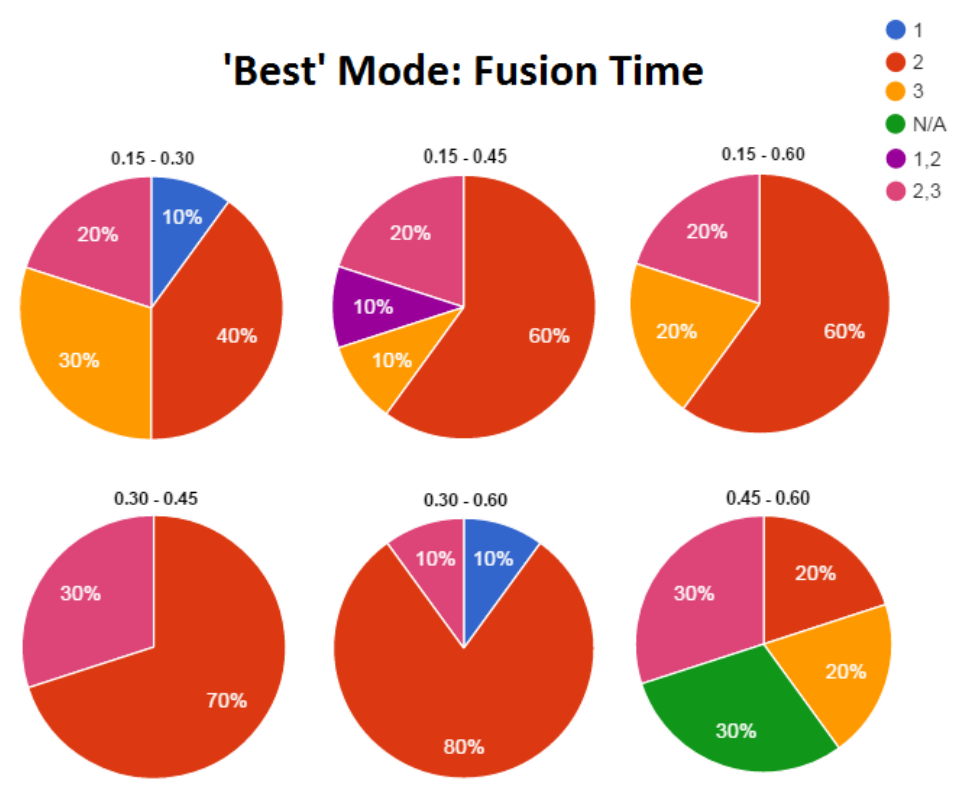

Figure 6.15: The number of participants that thought which mode(s) took the shortest amount of time to fuse with.

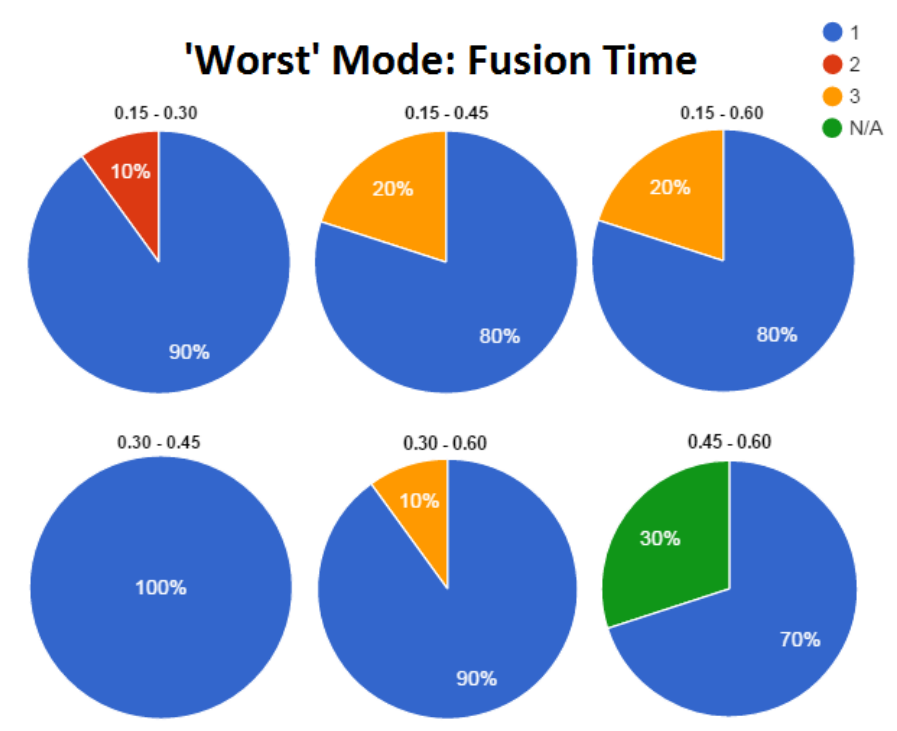

Figure 6.16: The number of participants that thought which mode(s) took the longest amount of time to fuse with. 


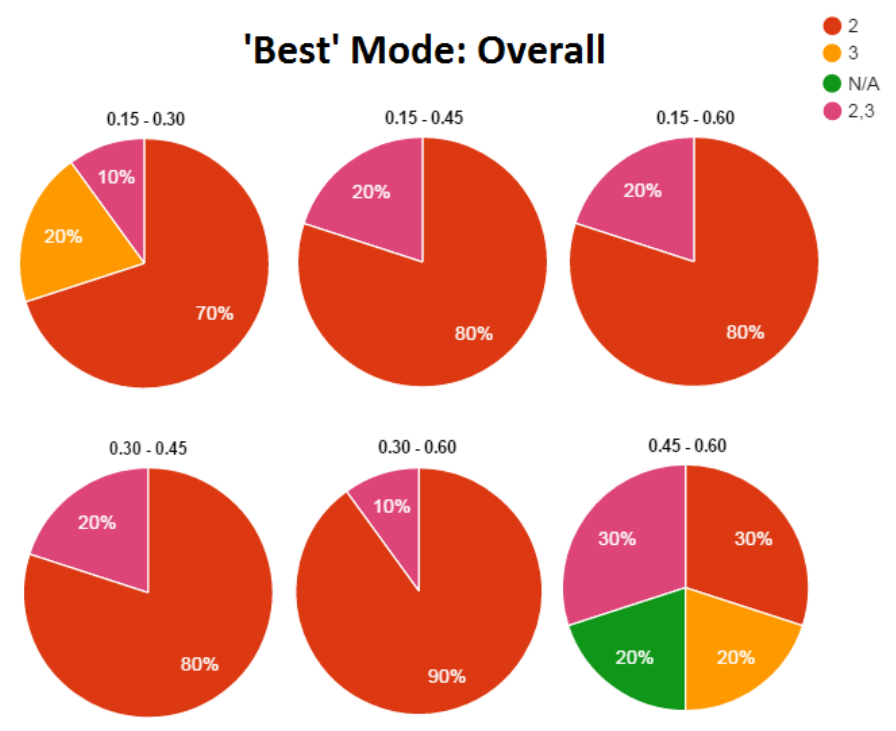

Figure 6.17: The number of participants that thought which mode(s) were overall, the best to use.

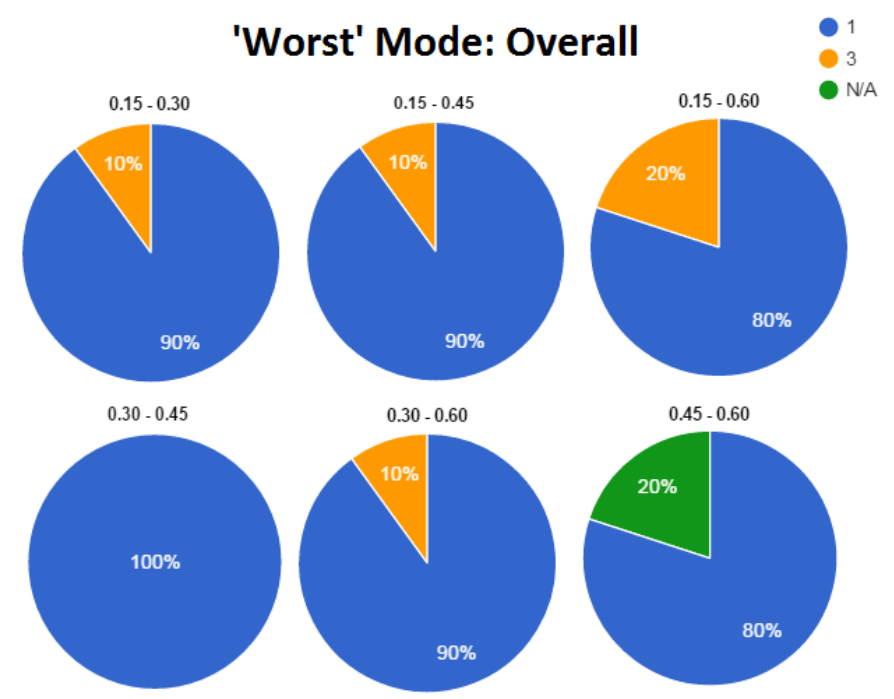

Figure 6.18: The number of participants that thought which mode(s) were overall, the worst to use. 


\subsubsection{Continuous Movement}

Figures 6.19 through 6.25 illustrate the voted 'best' modes in terms of different categories, when looking at a close virtual object that moves over a period of time. The 'worst' mode can be interpreted by taking the inverse of the results.

For the following graphs, the notations mean:

- 1 - No DC.

- 2 - DC with No Interpolation.

- N/A - No difference between the modes.

In terms of Nausea (Figure 6.21) and Dizziness (Figure 6.22), a good majority of participants $(60 \%$ to $80 \%)$ did not notice any difference. Those that did notice a difference thought that Mode 2 was the best mode.

For the rest of the categories, Mode 2 had upwards of $60 \%$ or more of the participants thinking it was the best mode, with unanimous preference when the object was moving at its Fast speed. Mode 1 was rarely chosen as the 'best' mode. 


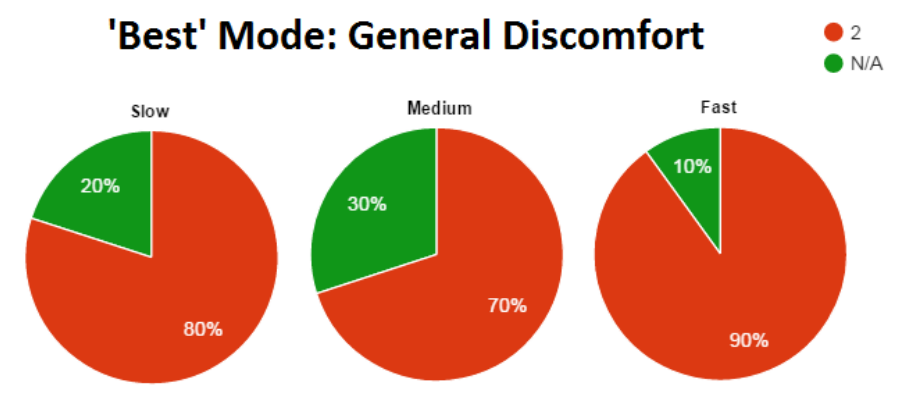

Figure 6.19: The number of participants that thought which mode(s) gave the least amount of general discomfort.

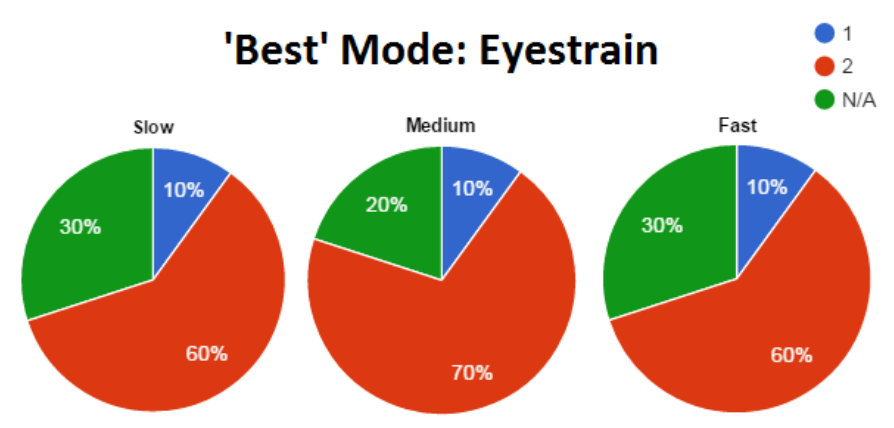

Figure 6.20: The number of participants that thought which mode(s) gave the least amount of eyestrain.

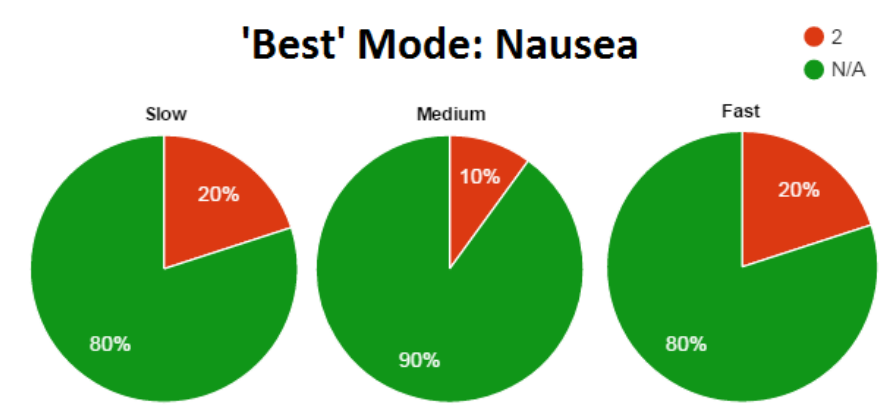

Figure 6.21: The number of participants that thought which mode(s) gave the least amount of nausea. 


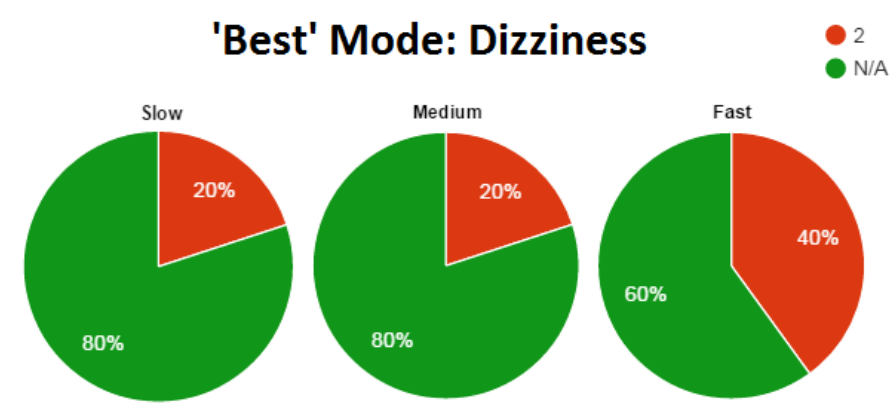

Figure 6.22: The number of participants that thought which mode(s) gave the least amount of dizziness.

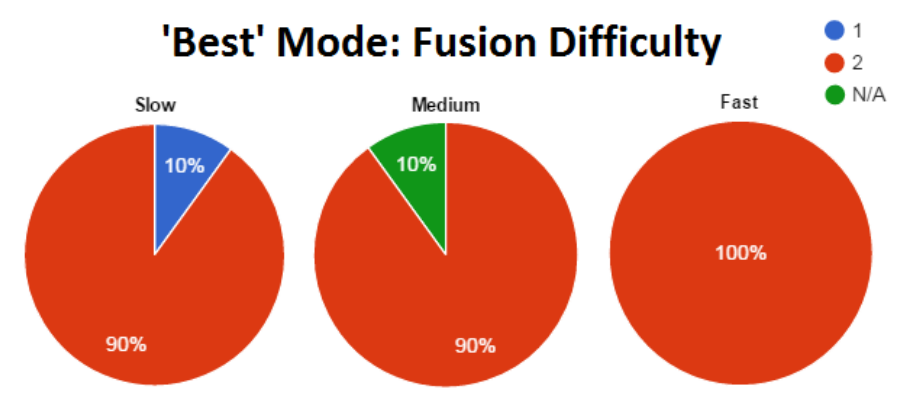

Figure 6.23: The number of participants that thought which mode(s) was the easiest to fuse with.

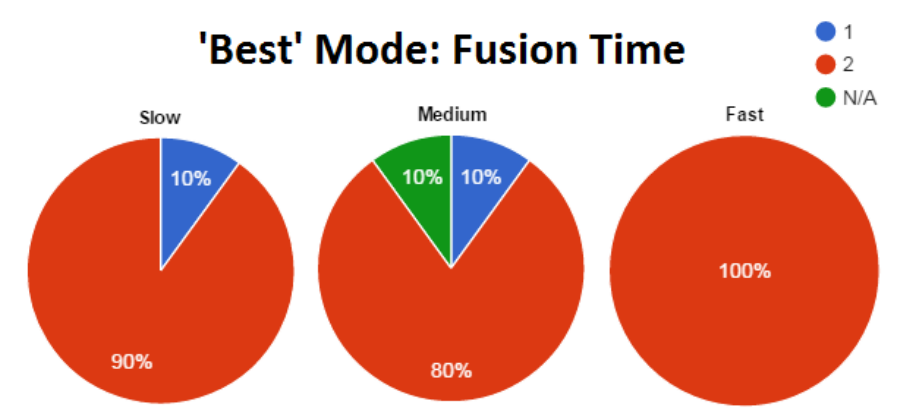

Figure 6.24: The number of participants that thought which mode(s) took the shortest amount of time to fuse with. 


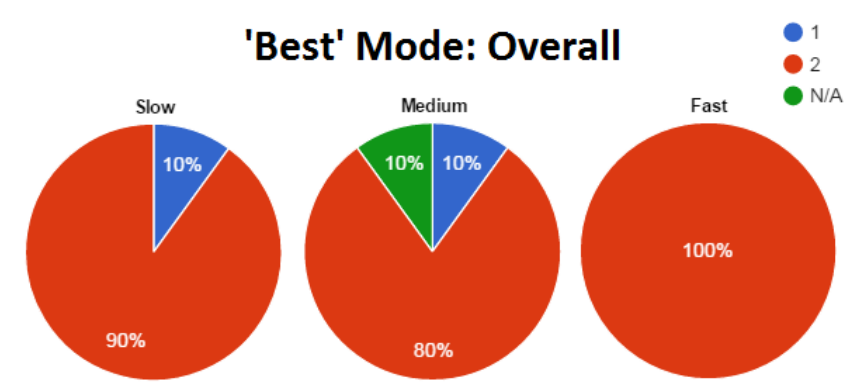

Figure 6.25: The number of participants that thought which mode(s) were overall, the best to use.

\subsubsection{Summary}

From the results of this user study, it was found that:

1. DC without interpolation was shown to be the most preferred in terms of multiple categories for both discrete and continuous object movement.

2. DC with interpolation however, had very mixed preferences between participants.

3. No DC was shown to be the least preferred mode in the majority of cases.

\subsection{Discussion}

The results shown here back up the findings of the first user test in terms of DC being effective for helping binocular fusion at the closer distances of $0.3 \mathrm{~m}$ or less. The results of the second user test also show that without some form of DC, participants tended to experience more discomfort symptoms. The opinion for DC with 'No Interpolation' remained high 
for both discrete movement and continuous movement of a virtual object. This supports DC's importance when viewing both a point of interest at a short vergence depth and a change in vergence depth that involves a short depth.

For the distance scenario $0.45 \mathrm{~m}-0.6 \mathrm{~m}$, there were mixed opinions and the increased number of participants noticing no difference between the modes, this was likely due to a diminished effect that DC would have at those distances due to a low vergence angle. However, it was noted that there were a few cases of DC not being preferred, which could mean that DC may not be suited for everyone. 


\section{Chapter 7}

\section{Conclusion and Discussion}

This research has adopted a mixed experimental approach in order to add to the growing body of research and knowledge that can perhaps be used to help solve the AVC problem, which is potentially holding back the widespread use of HMDs. Through the use of software, by enhancing the DC functionality within the Unity3D game engine and by conducting user experiments and surveys, both quantitative and qualitative statistical techniques have been used in order to measure and investigate in some detail the scale of the problem associated with the impact of DC on the human visual system in HMDs. In particular, the effects of DC when viewing close virtual objects. In this chapter, following a review of the problem, a recap of the software solution is provided before a summary of the user tests and the results of the analysis. Finally, the limitations of this DC implementation are also pointed out along with a discussion on possible future work that may either addresses these limitations or expand the results of this research. 


\subsection{Summary}

Due to the adverse effects from the AVC, it is important to have correct or consistent accommodation and vergence cues for comfortable 3D viewing and to avoid health risks on the human visual system. However with the current lack of flexible vergence in HMD-based VR systems, any virtual objects that are in front of the focal plane set by the predefined vergence distance will have an incorrect vergence cue. Thus all objects at close range are often viewed under double vision and any stereo vision that can be achieved using binocular fusion can be easily broken. This has a severe effect on a user's immersion of the VE when trying to interact or view close objects.

A DC system was designed with the assumption that the centre of the virtual viewport of the Oculus corresponded with the user's gaze in determining the vergence depth the virtual cameras were rotated for. Different transitions for a change in vergence depth were proposed in order to find which was more beneficial for a user's comfort. This was then implemented in Unity3D and tested in user studies in order to measure any symptoms of discomfort and evaluate its aid in helping binocular fusion.

For stationary close virtual objects, using in-depth quantitative statistical analysis DC was found to be effective in reducing the effort required for binocular fusion at a vergence depth of $0.15 \mathrm{~m}$, while having no notable negative impact on a user's visual discomfort. At this depth there was demonstrable statistical significance which gives the results some credibility for scientific tests. However, there was also some evidence that at a depth of $0.3 \mathrm{~m}$ there were improvements with DC enabled as found by a third of the participants, although this test did not pass the the selected statistical robustness test like the shorter distance. Similarly, for a change in vergence depth (both instantaneous and peri- 
odic), with DC enabled the system allowed participants to perform binocular fusion easier at the closer distance of $0.15 \mathrm{~m}$. It was also promising that the majority of users did not experience any greater discomfort with these improvements.

A transition for interpolating the change in vergence depth was also investigated. The results of the quantitative analysis for the experimental tests using three different interpolation techniques across six distances found little strength in being able to formulate an argument in favour of any of the three interpolation techniques reviewed (no interpolation, linear interpolation, ease interpolation). The majority of tests recorded very little change at the low end of the discomfort and ease of difficultly. There was however, a small increase in eyestrain which whilst not deemed statistically significant here, may well warrant further testing and consideration in future studies.

Further to the basic questionnaire for the change in vergence depth there was also a qualitative component to the research with the intention of finding out exactly which setting the participants most preferred. The findings from this were that there was a strong unanimous dislike for the ease interpolation and split results between linear and no interpolation.

The qualitative data collected in the second user evaluation back up the findings of the first evaluation in terms of DC being effective for helping binocular fusion at the closer distances of $0.30 \mathrm{~m}$ or less. In addition, without some form of DC, participants tended to experience more discomfort symptoms. In terms of having a transition, DC with No Interpolation was shown to be the most preferred in terms of multiple categories. While, DC with Linear Interpolation however has very mixed preferences.

Whilst both user evaluations reported mixed preferences for the 'Linear Interpolation' and 'No Interpolation', these results conclude that 
there may not be a definitive transition for a change in vergence depth, but rather a personal preference for one. Thus there needs to be flexibility in future work and implementations of DC to include the option of enabling and disabling a transition for its users.

\subsection{Limitations and Future Work}

The survey based approach used here is similar to other researcher's methodology in attempting to measure users' perception of stereoscopic viewing by experiment, indeed, it falls between what Kramida and Varshney refer to as a performance-oriented and appreciation-oriented study whereby users were asked to perform various visual activities and give their subjective opinion of their viewing experience [26]. As the researchers point out whilst user studies of this kind are widely accepted and popular for such evaluations, the results are prone to numerous pitfalls. The research here is no different in this regard and therefore carries the same caveats regarding its drawbacks and shortcomings which include issues such as problems with quantification, the subjectiveness of the measured responses, user bias and limited population sample sizes.

A strong emphasis here has been placed on quantitative analysis. With its objectivity, quantitative methods are replicable with precision and allow control along with the ability to conduct sophisticated analyses through well-defined statistical techniques. Coupled with the fact that for scientific research to be useful, not only does it require defendable and well understood statistical techniques, it also requires probabilistic statistical inferences that can be applied to the broader population and thus provide a useful contribution to the research body of knowledge. However, it can also be argued that the adoption of the commonly used statistical probability threshold of 0.05 used here as a measure of evidence against a 
null effect is somewhat arbitrary, and "black or white", particularly when used in relatively small sample surveys that involve the "grey area" of people based experiments. For example, probability is based on mathematical chance, which is perhaps not the most important item to measure where people are concerned, and this may lead to the search for margins of error in the mathematics rather than perhaps looking at how the questions of the survey where asked and how well they were understood [12]. Since due to human agency people are not inert objects of the kind typically measured in the physical sciences [20] and thus will respond quite differently and unpredictably. It was for this reason and at the risk of some subjectivity, that the qualitative methods and analysis was also included.

It is also worth pointing out that there were some potential weaknesses with the sample demographics. As previously mentioned in Section 5.1.1 there was a lack of variety in age (range 17-36) for the study. Visual performance is known to change with age, so the findings of this study are mainly relevant to young adults only.

To avoid potential invalid results, the study disallowed participants with abnormal eye conditions. In Section 5.2.2 a result regarding a participant who wore bifocal glasses was discussed. It was discovered that DC had a negative impact for this participant, showing that DC may not be suited for all people due to various eye conditions. Further research could investigate the effect that certain eye conditions have for viewing VR content with and without DC.

The gaze estimation algorithm used in the implementation did not work as intended if the assumption of the user looking at the centre of the screen is broken. This was encountered during the study and discussed in Section 5.2.2 where a result from a participant was discarded due to the broken assumption. The gaze estimation could be improved with a better software solution or the introduction of new hardware. An ex- 
ample of such hardware would be an eye-tracker that would be built into the HMD, this would give accurate information on the current point of interest in the virtual scene and thus the correct vergence depth. If an eyetracker is used however, the implementation of DC would have to change greatly, as the orientation of the user's eyes would not be guaranteed to be looking straight ahead for the object of interest. This would mean that the correct virtual camera rotation would be a lot more complex and nonsymmetrical. There could also be a feedback loop between virtual camera rotation and eye-tracking, as a camera rotation may change the position of where the user is looking, which would then change the camera rotation; and continue the loop. Further research into the use of eye-tracking and vergence would have to overcome this problem.

The distances that were investigated in this research were only in $0.15 \mathrm{~m}$ increments. Further research may wish to investigate in greater detail the exact vergence depth that users have difficulty with binocular fusion in HMDs. Furthermore, there were a few participants from the user studies that had trouble performing binocular fusion at a distance of $0.30 \mathrm{~m}$ and even $0.45 \mathrm{~m}$. This may be due to factors not recorded by this research, further work could investigate how age, eye conditions and other possible variables have an effect on binocular fusion difficulty in HMDs.

\subsection{Conclusion}

User experiments to investigate the impact of DC on visual discomfort and binocular fusion on HMDs such as the Oculus Rift DK2 was performed. It was found that the DC was effective at reducing the difficulty of performing binocular fusion of virtual objects at a vergence depth of approximately $0.3 \mathrm{~m}$ or less (with statistical significance confirmed at $0.15 \mathrm{~m}$ ), while not having any negative effect on visual discomfort. In addition, the DC 
system helped binocular fusion occur after any change in vergence depth that involved the $0.15 \mathrm{~m}$ mark. This highlights the need of assistance for performing binocular fusion when viewing virtual objects in front of, or near, a vergence depth of approximately $0.15 \mathrm{~m}$ to $0.3 \mathrm{~m}$. Furthermore, a number of users felt the need of a transition, such as 'Linear Interpolation', between vergence depths while using DC, however this had a negative effect on other users. Thus there needs to be the option of enabling and disabling a transition for DC on user demand. Future applications of these results include more comfortable and realistic viewing of close up interactive VR content, such as films and games on HMDs. 


\section{Bibliography}

[1] Akeley, K., Watt, S. J., Girshick, A. R., and BanKs, M. S. A stereo display prototype with multiple focal distances. In ACM transactions on graphics (TOG) (2004), vol. 23, ACM, pp. 804-813.

[2] Ballard, D. H., AND HayHoE, M. M. Modelling the role of task in the control of gaze. Visual cognition 17, 6-7 (2009), 1185-1204.

[3] BANDO, T., IIjIMA, A., AND YANO, S. Visual fatigue caused by stereoscopic images and the search for the requirement to prevent them: A review. Displays 33, 2 (2012), 76-83.

[4] Bernhard, M., Dell'mour, C., Hecher, M., Stavrakis, E., AND WIMMER, M. The effects of fast disparity adjustment in gazecontrolled stereoscopic applications. In Proceedings of the Symposium on Eye Tracking Research and Applications (2014), ACM, pp. 111-118.

[5] Bharadwaj, S. R., AND CANDY, T. R. Accommodative and vergence responses to conflicting blur and disparity stimuli during development. Journal of vision 9, 11 (2009), 4-4.

[6] BiaU, D. J., Jolles, B. M., AND PORCher, R. P value and the theory of hypothesis testing: an explanation for new researchers. Clinical Orthopaedics and Related Research ${ }^{\circledR}$ 468, 3 (2010), 885-892. 
[7] Biguer, B., Jeannerod, M., And Prablanc, C. The coordination of eye, head, and arm movements during reaching at a single visual target. Experimental Brain Research 46, 2 (1982), 301-304.

[8] BiochemiA-MedicA. The selection process for the right statistical test, 2010. [Online; accessed December 28, 2016].

[9] BRILL, L. Metaphors for the traveling cybernaut. Virtual Reality World 1,1 (1993).

[10] CARnegie, K. Mitigating visual discomfort on head mounted displays using estimated gaze dependent depth of field.

[11] Celikcan, U., Cimen, G., Kevinc, E. B., And Capin, T. Attentionaware disparity control in interactive environments. The Visual Computer 29, 6-8 (2013), 685-694.

[12] DAhiru, T. P-value, a true test of statistical significance? a cautionary note. Annals of Ibadan postgraduate medicine 6, 1 (2008), 21-26.

[13] Dichgans, J., AND BRANDT, T. Visual-vestibular interaction: Effects on self-motion perception and postural control. In Perception. Springer, 1978, pp. 755-804.

[14] Fincham, E., AND WALTON, J. The reciprocal actions of accommodation and convergence. The Journal of Physiology 137, 3 (1957), 488.

[15] Fisker, M., Gram, K., Thomsen, K. K., Vasilarou, D., And KRAUS, M. Automatic convergence adjustment for stereoscopy using eye tracking. In Eurographics (Posters) (2013), pp. 23-24.

[16] HeILIG, M. Stereoscopic-television apparatus for individual use, Oct. 4 1960. US Patent 2,955,156.

[17] Hettinger, L. J., Berbaum, K. S., Kennedy, R. S., Dunlap, W. P., AND Nolan, M. D. Vection and simulator sickness. Military Psychology 2, 3 (1990), 171. 
[18] Howard, I. P., AND Rogers, B. J. Seeing in depth, volume 2: Depth perception. Ontario, Canada: I. Porteous (2002).

[19] HowARTH, P., AND Costello, P. The occurrence of virtual simulation sickness symptoms when an hmd was used as a personal viewing system. Displays 18, 2 (1997), 107-116.

[20] Hughes, C. Qualitative and quantitative approaches to social research. Coventry: Department of Sociology, Warwick University.[Accessed 29 October 2013] (2006).

[21] JACOBSON, L. Welcome to the virtual world. On the cutting edge of technology (1993), 69-79.

[22] Johnson, D. M. Introduction to and review of simulator sickness research. Tech. rep., DTIC Document, 2005.

[23] Kennedy, R. S., Lane, N. E., Berbaum, K. S., and Lilienthal, M. G. Simulator sickness questionnaire: An enhanced method for quantifying simulator sickness. The international journal of aviation psychology 3, 3 (1993), 203-220.

[24] Kenny, A., Koesling, H., Delaney, D., Mcloone, S., And WARD, T. A preliminary investigation into eye gaze data in a first person shooter game.

[25] Kim, J., Chung, C. Y., Nakamura, S., Palmisano, S., and KHUU, S. K. The oculus rift: a cost-effective tool for studying visualvestibular interactions in self-motion perception. Frontiers in psychology 6 (2015).

[26] KRAMIDA, G. Resolving the vergence-accommodation conflict in head-mounted displays. IEEE transactions on visualization and computer graphics 22, 7 (2016), 1912-1931. 
[27] LiU, S., HuA, H., AND CHENG, D. A novel prototype for an optical see-through head-mounted display with addressable focus cues. IEEE transactions on visualization and computer graphics 16, 3 (2010), 381-393.

[28] Love, G. D., Hoffman, D. M., HAnds, P. J., GaO, J., Kirby, A. K., AND BANKS, M. S. High-speed switchable lens enables the development of a volumetric stereoscopic display. Optics express 17, 18 (2009), 15716-15725.

[29] Mantiuk, R., Bazyluk, B., And TomaszewskA, A. Gazedependent depth-of-field effect rendering in virtual environments. In International Conference on Serious Games Development and Applications (2011), Springer, pp. 1-12.

[30] Martens, T. G., AND OGLE, K. N. Observations on accommodative convergence; especially its nonlinear relationships. American journal of ophthalmology 47, 1 Pt 2 (1959), 455-62.

[31] McCAuley, M. E. Research issues in simulator sickness: Proceedings of a workshop. National Academies, 1984.

[32] Miller, J., AND GoOdsON, J. A note concerning motion sickness in the $2 \mathrm{fh} 2$ hover trainer. Pensacola, FL: Naval School of Aviation Medicine (1958).

[33] Oskam, T., Hornung, A., Bowles, H., Mitchell, K., AND Gross, M. H. Oscam-optimized stereoscopic camera control for interactive 3d. ACM Trans. Graph. 30, 6 (2011), 189.

[34] Prothero, J. D., Parker, D. E., Furness, T., And Wells, M. Towards a robust, quantitative measure for presence. In Proceedings of the conference on Experimental Analysis and Measurement of Situation Awareness (1995), pp. 359-366. 
[35] Reichelt, S., HäUssler, R., Fütterer, G., AND Leister, N. Depth cues in human visual perception and their realization in $3 \mathrm{~d}$ displays. In SPIE Defense, Security, and Sensing (2010), International Society for Optics and Photonics, pp. 76900B-76900B.

[36] Rothkopf, C. A., Ballard, D. H., And Hayhoe, M. M. Task and context determine where you look. Journal of vision 7, 14 (2007), 16-16.

[37] Rushton, S. K., AND Riddell, P. M. Developing visual systems and exposure to virtual reality and stereo displays: some concerns and speculations about the demands on accommodation and vergence. Applied Ergonomics 30, 1 (1999), 69-78.

[38] Sherstyuk, A., Dey, A., SAndor, C., ET Al. Dynamic eye convergence for head-mounted displays improves user performance in virtual environments. In Proceedings of the ACM SIGGRAPH Symposium on Interactive 3D Graphics and Games (2012), ACM, pp. 23-30.

[39] Sherstyuk, A., ET AL. Dynamic eye convergence for head-mounted displays. In Proceedings of the 17th ACM Symposium on Virtual Reality Software and Technology (2010), ACM, pp. 43-46.

[40] Shibata, T., Kim, J., Hoffman, D. M., And Banks, M. S. The zone of comfort: Predicting visual discomfort with stereo displays. Journal of vision 11, 8 (2011), 11-11.

[41] Slater, M. Place illusion and plausibility can lead to realistic behaviour in immersive virtual environments. Philosophical Transactions of the Royal Society of London B: Biological Sciences 364, 1535 (2009), 3549-3557.

[42] Stanney, K. M., Hale, K. S., Nahmens, I., And Kennedy, R. S. What to expect from immersive virtual environment exposure: Influences of gender, body mass index, and past experience. Human 
Factors: The Journal of the Human Factors and Ergonomics Society 45, 3 (2003), 504-520.

[43] State, A., Ackerman, J., Hirota, G., Lee, J., And Fuchs, H. Dynamic virtual convergence for video see-through head-mounted displays: maintaining maximum stereo overlap throughout a closerange work space. In Augmented Reality, 2001. Proceedings. IEEE and ACM International Symposium on (2001), IEEE, pp. 137-146.

[44] Stern, R. M., Hu, S., Anderson, R. B., Leibowitz, H. W., AND $\mathrm{KOCH}, \mathrm{K}$. L. The effects of fixation and restricted visual field on vection-induced motion sickness. Aviation, Space, and Environmental Medicine (1990).

[45] Stidwill, D., AND Fletcher, R. Normal binocular vision: Theory, investigation and practical aspects. John Wiley \& Sons, 2010.

[46] VIIRRE, E. A survey of medical issues and virtual reality technology. Virtual Reality World 2, 4 (1994), 16. 
Appendix A

Code of the EGD-IDC System 


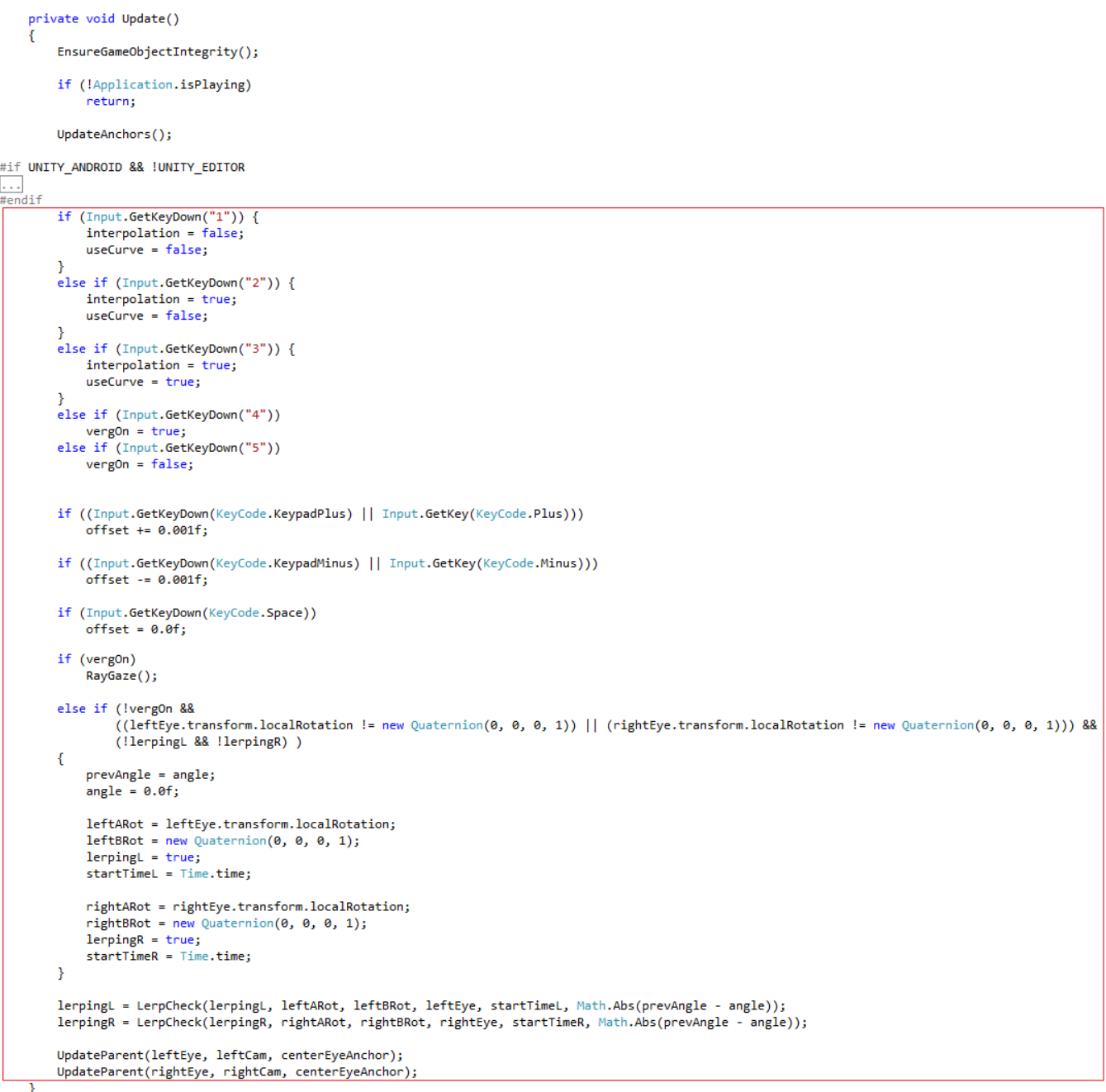

Figure A.1: The Update function which executes every frame, the red box highlights what code was added to this function in the modified script OVRCameraRig.cs 


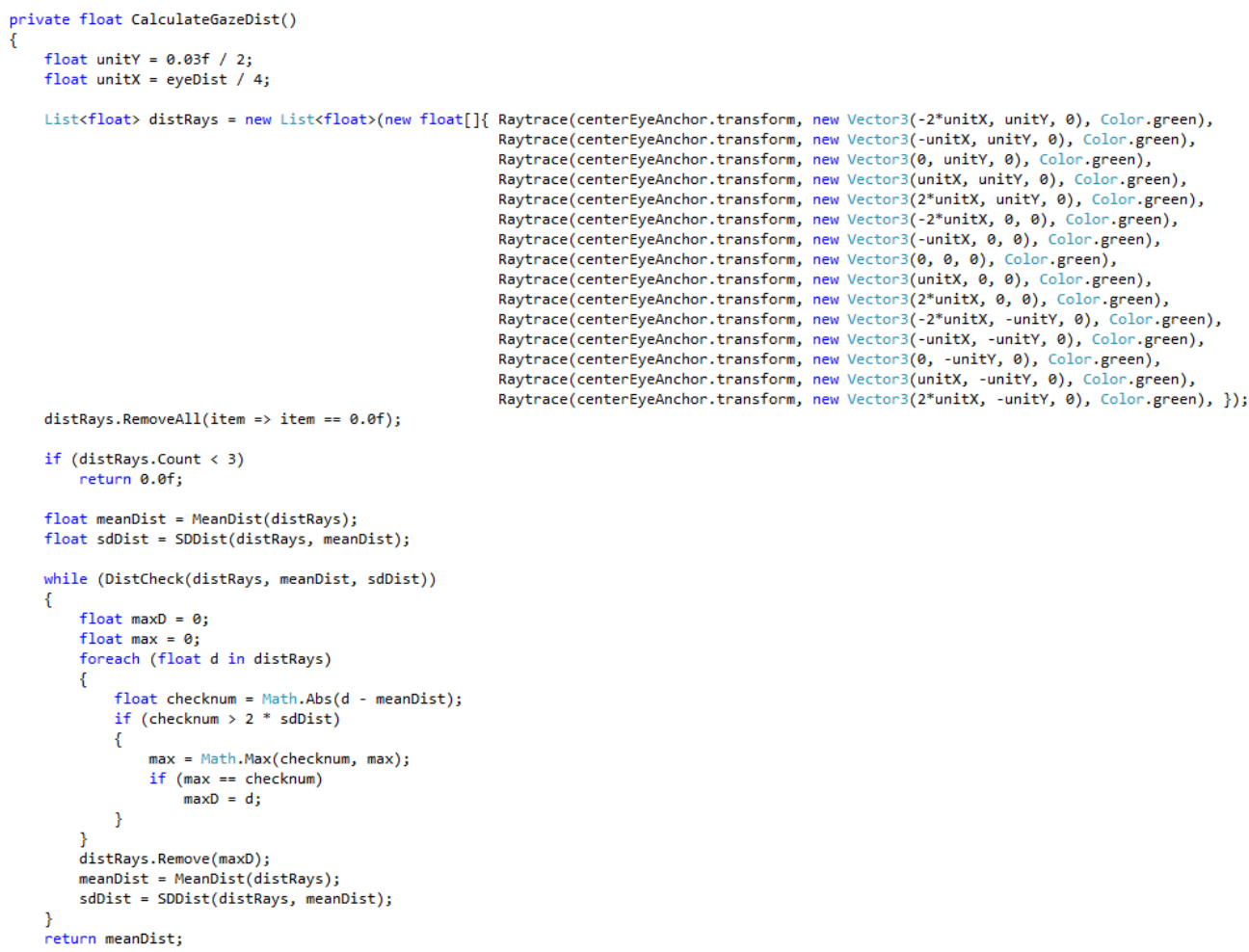

Figure A.2: Code for the Calculating the Vergence Distance based on the Estimated Gaze Assumption 


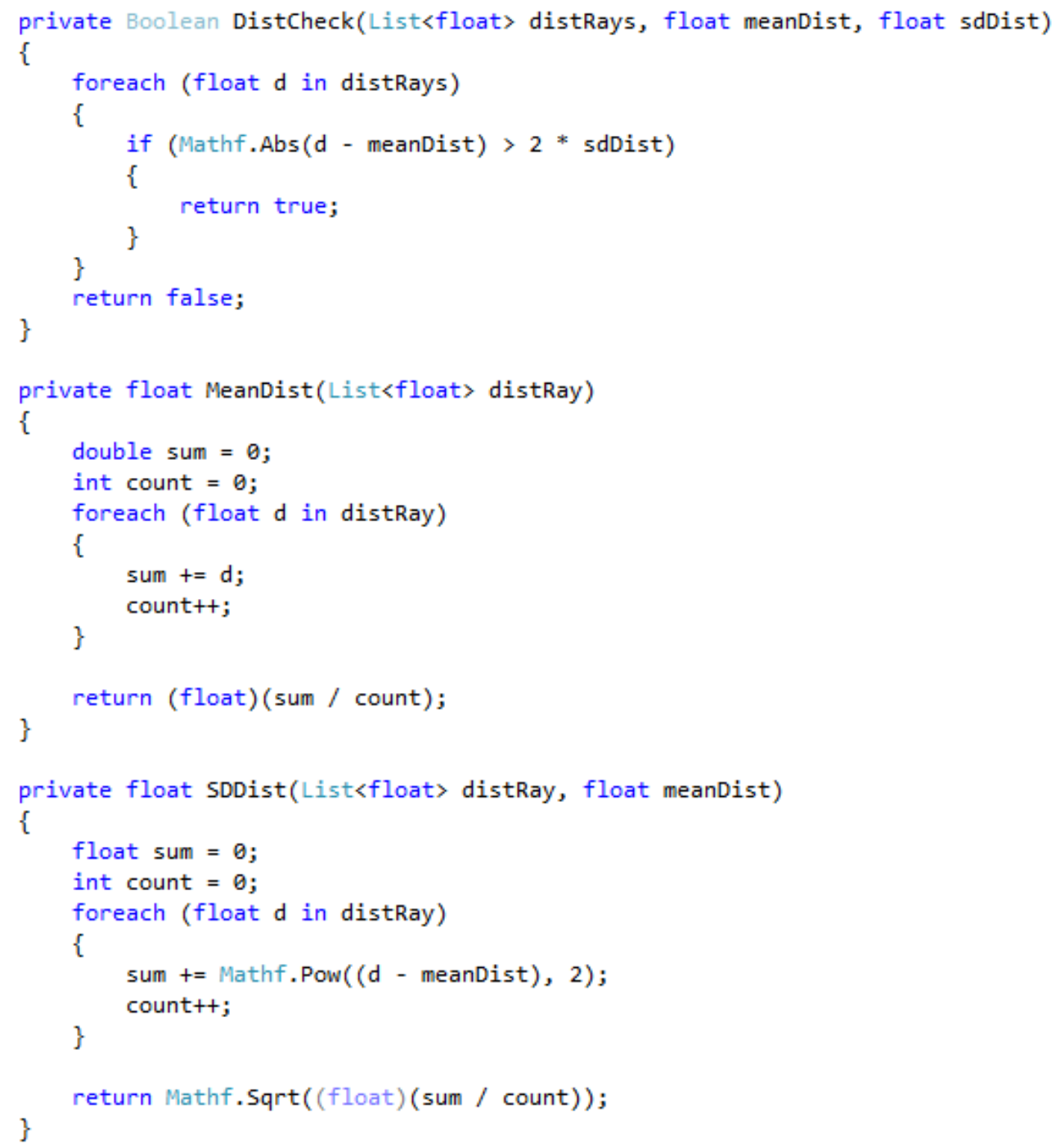

Figure A.3: Other math functions CalculateGazeDist() uses 


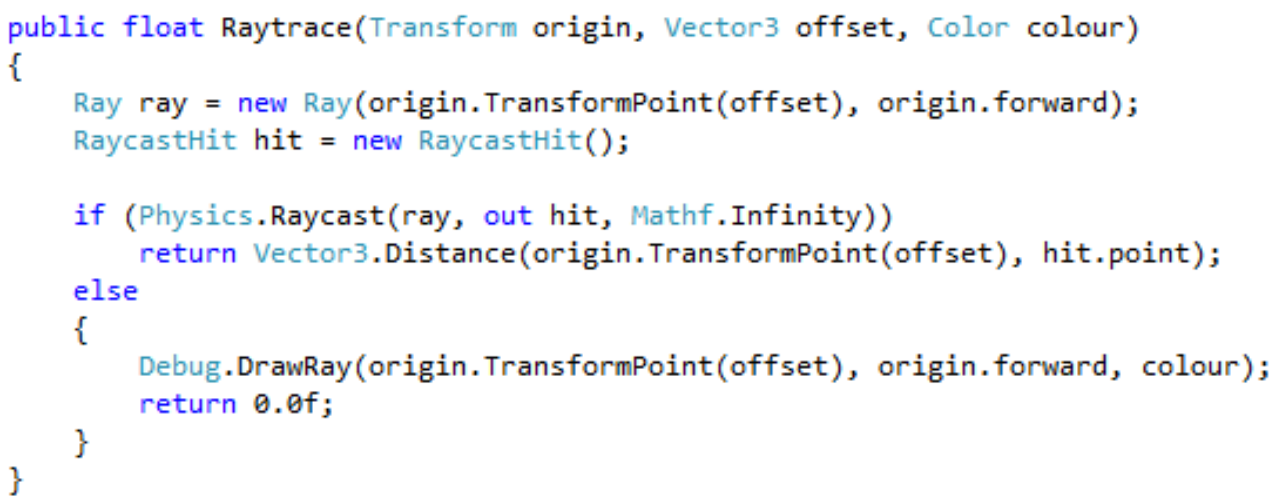

Figure A.4: Code for handling the Raytracing in Unity3D

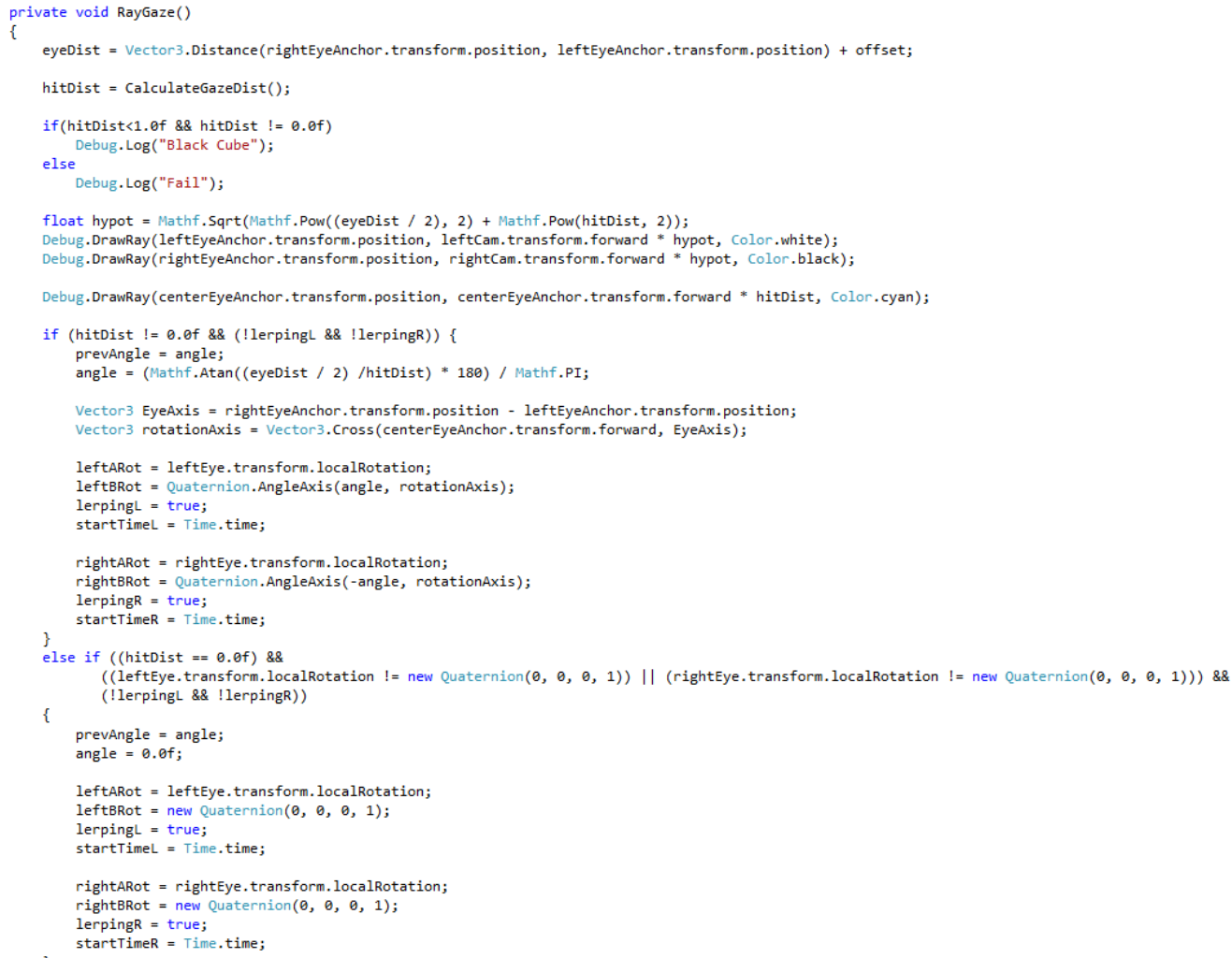

Figure A.5: Code for handling starting and finishing angles of the virtual cameras 


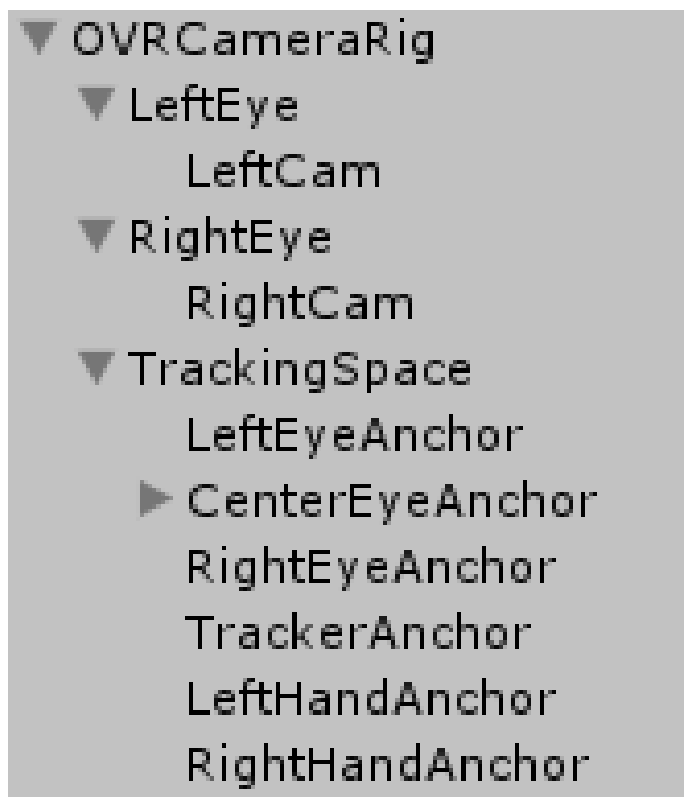

Figure A.6: The hierarchical setup used in the Unity3D Editor

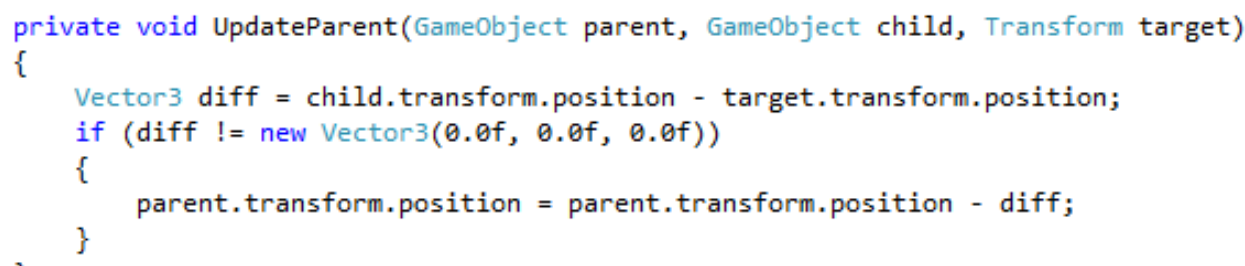

Figure A.7: Used to update the position of the virtual eyes 


\begin{tabular}{|c|c|c|c|c|c|c|c|}
\hline \multirow{3}{*}{$\begin{array}{l}\nabla \text { G } \square \text { Camera } \\
\text { Clear Flags } \\
\text { Background }\end{array}$} & \multicolumn{3}{|c|}{ [1] } & \multirow{3}{*}{$\begin{array}{l}\text { Clear } \square \text { Camera } \\
\text { Clear Flags } \\
\text { Background }\end{array}$} & \multicolumn{3}{|r|}{ [a] } \\
\hline & \multicolumn{3}{|c|}{ Skybox } & & \multicolumn{3}{|c|}{ Skybox } \\
\hline & \multicolumn{3}{|c|}{ 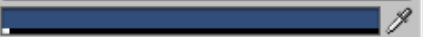 } & & & & 8 \\
\hline Culling Mask & \multicolumn{3}{|c|}{ Everything } & Culling Mask & \multicolumn{3}{|c|}{ Everything } \\
\hline Projection & \multicolumn{3}{|c|}{ Perspective } & Projection & \multicolumn{3}{|c|}{ Perspective } \\
\hline Field of View & \multicolumn{3}{|c|}{$\longrightarrow \longdiv { 1 0 6 . 1 8 \varepsilon }$} & Field of View & \multicolumn{3}{|c|}{$\longrightarrow \quad 106.18 \varepsilon$} \\
\hline \multirow[t]{2}{*}{ Clipping Planes } & \multicolumn{3}{|c|}{ Near 0.01} & \multirow[t]{2}{*}{ Clipping Planes } & \\
\hline & Far & 1000 & & & Far & 1000 & \\
\hline \multirow[t]{2}{*}{ Viewport Rect } & $\times 0$ & Y 0 & & \multirow[t]{2}{*}{ Viewport Rect } & $x 0$ & $Y 0$ & \\
\hline & $w \longdiv { 1 }$ & $\mathrm{H} 1$ & & & $w \longdiv { 1 }$ & $\mathrm{H} 1$ & \\
\hline Depth & \multicolumn{3}{|l|}{0} & Depth & \multicolumn{3}{|l|}{0} \\
\hline Rendering Path & \multicolumn{3}{|c|}{ Use Player Settings } & Rendering Path & \multicolumn{3}{|c|}{ Use Player Settings } \\
\hline Target Texture & \multicolumn{3}{|c|}{ None (Render Texture) } & Target Texture & \multicolumn{3}{|c|}{ None (Render Texture) } \\
\hline Occlusion Culling & \multicolumn{3}{|l|}{$\nabla$} & Occlusion Culling & \multicolumn{3}{|l|}{$\nabla$} \\
\hline HDR & \multicolumn{3}{|l|}{$\square$} & HDR & \multicolumn{3}{|l|}{$\square$} \\
\hline Target Display & \multicolumn{3}{|c|}{ Display 1} & Target Display & \multirow{2}{*}{\multicolumn{3}{|c|}{ Display 1}} \\
\hline Target Eye & \multicolumn{3}{|l|}{ Left } & Target Eye & & & \\
\hline
\end{tabular}

Figure A.8: Left: the camera component of LeftCam. Right: the camera component of RightCam

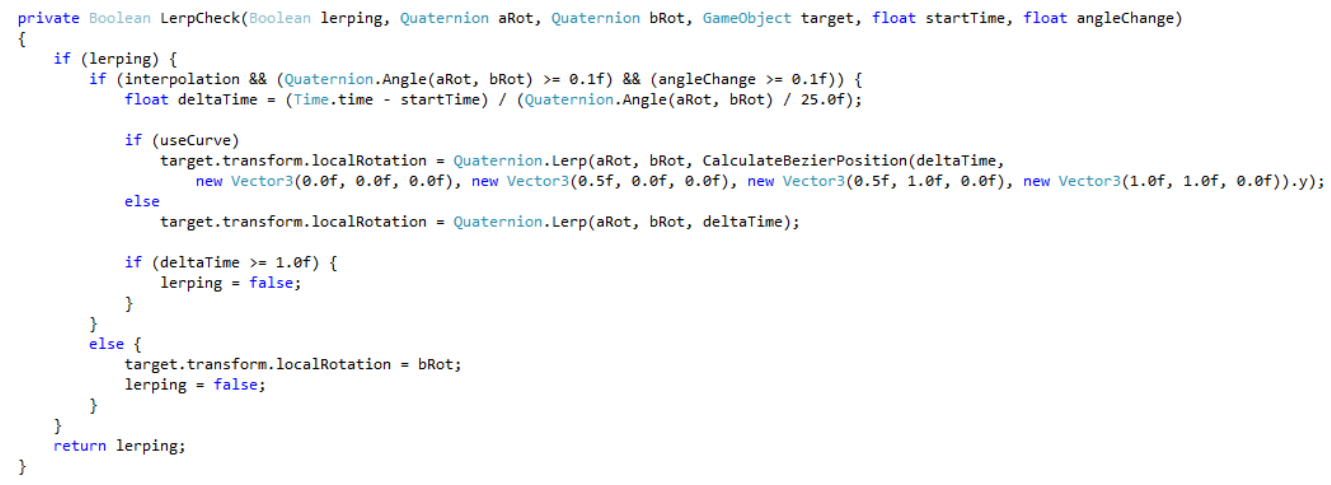

Figure A.9: Handles the interpolation of the virtual cameras

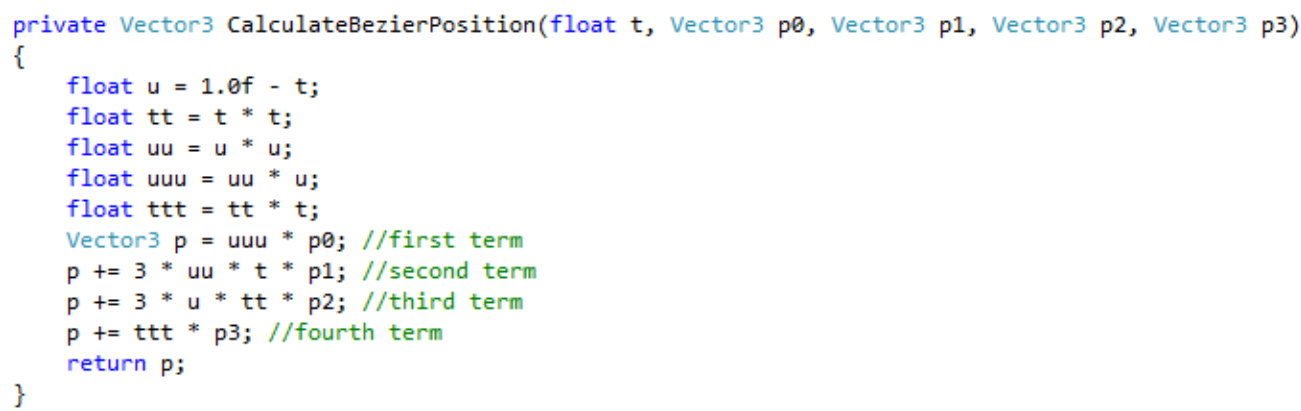

Figure A.10: Used to the wanted position on the beizer curve 
Appendix B

Advertisements, Forms and Questionnaires 


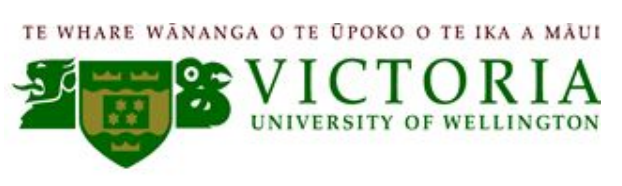

\section{Participate in a Computer Graphics Study}

We are conducting a study involving people's level of (dis)comfort when using the Oculus Rift. During two 45 minute sessions, you will experience a virtual world and answer some questions about the experience.

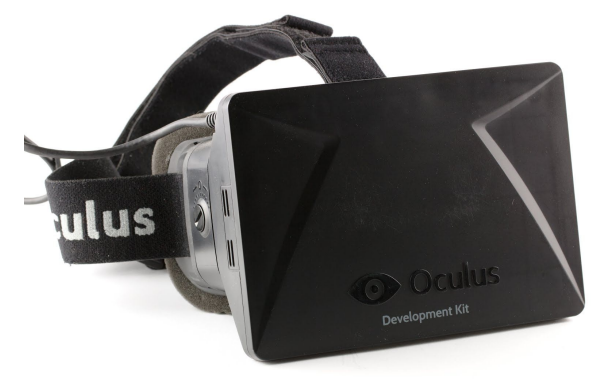

To participate you must:

- Not have any severe eye abnormalities (excluding being short/long sighted).

- Not be pregnant.

- Not be predisposed to motion sickness.

This research has VUW Human Ethics Committee (HEC) approval.

For more information please either phone me at +04-463 5233, extn 8286 OR e-mail me sumnerryan@ecs.vuw.ac.nz.

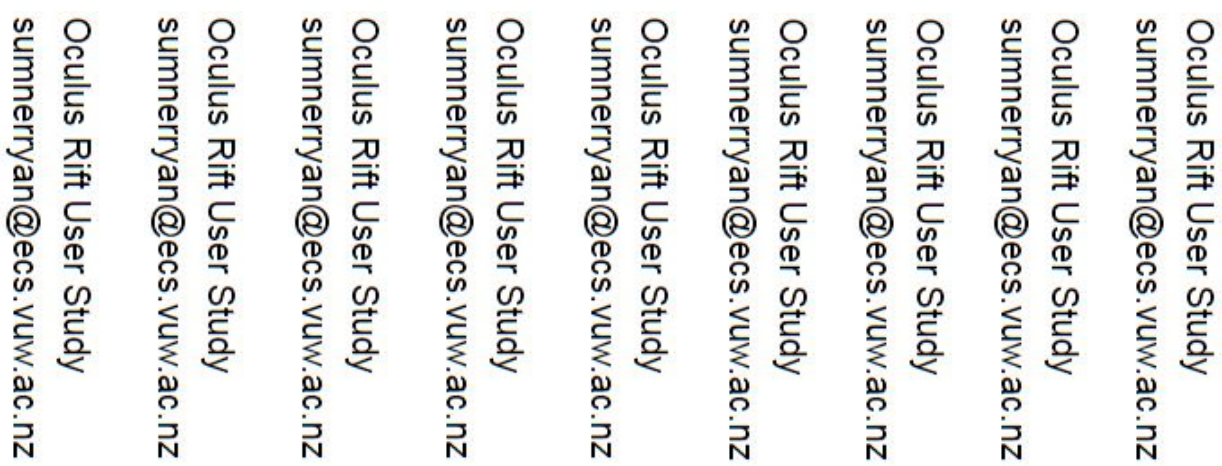

Figure B.1: The advertisement used to recruit participants of the first user study 


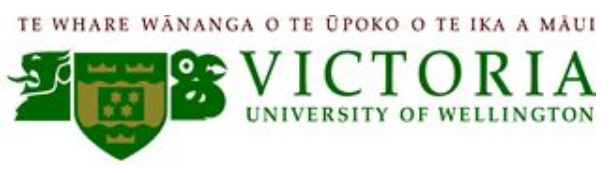

\section{Participate in a Computer Graphics Study}

We are conducting a study involving people's level of (dis)comfort when using the Oculus Rift. During a 45 minute session, you will experience a virtual world and answer some questions about the experience.

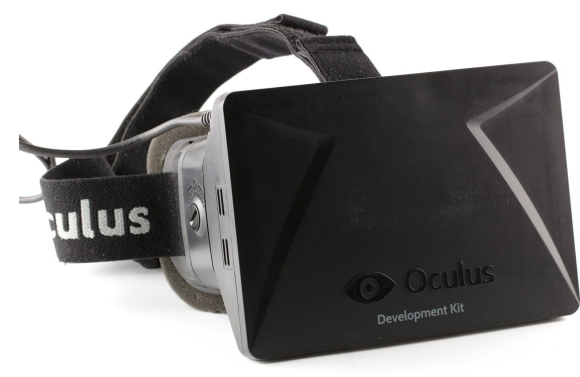

To participate you must:

- Not have any severe eye abnormalities (excluding being short/long sighted).

- Not be pregnant.

- Not be predisposed to motion sickness.

This research has VUW Human Ethics Committee (HEC) approval.

For more information please either phone me at +04-463 5233, extn 8286 OR e-mail me sumnerryan@ecs.vuw.ac.nz.

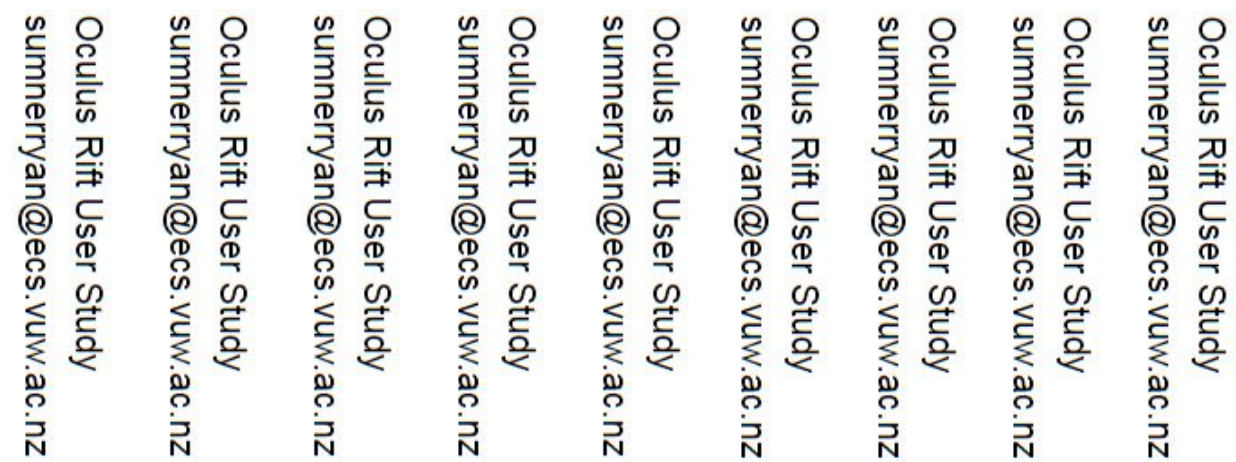

Figure B.2: The advertisement used to recruit participants of the second user study 


\section{APPENDIX B. ADVERTISEMENTS, FORMS AND QUESTIONNAIRES}

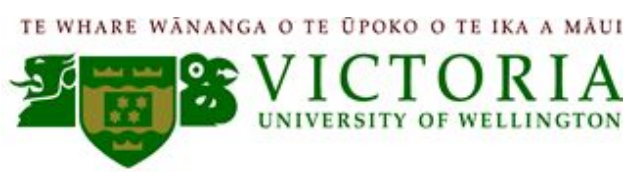

Investigating Discomfort in Head Mounted Displays

Ryan Sumner; Masters research project; Victoria University of Wellington

\section{Participant Consent form}

Please read the following notices. If you agree with them, please sign this form.

By signing this sheet: I, the participant, understand and agree that:

- My participation in this research is voluntary and I am aware that I am able to withdraw at any time.

- I am aware that, should I choose to withdraw, the investigator may ask me why I have chosen to withdraw but I am not compelled to answer if I do want to.

- I confirm that I have have been provided, read and understand the Participant Information Sheet.

- I have had the opportunity to ask any appropriate questions about this research and had them answered.

- I understand that all personal information will remain confidential and that all efforts will be made to ensure I cannot be identified (except as might be required by law).

- I agree that data gathered in this study will be anonymised and stored and securely, and may be used for future research.

- I agree to take part in this study.

- If I have any further concerns and/or questions, I am aware I can contact the investigator at: +04-463 5233, extn 8286 or via email at: sumnerryan@ecs.vuw.ac.nz I wish to receive a copy of any aggregate results of this study:

Yes

No

Participant's name \& signature:

Investigator's signature:

Date:

Figure B.3: The consent form participants signed for both user studies 


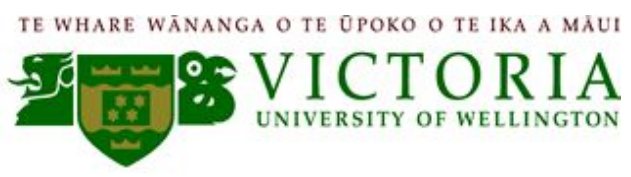

Investigating Discomfort in Head Mounted Displays

Ryan Sumner; Masters research project; Victoria University of Wellington

Participant Name:

Gender:

Age:

Questionnaire

SYMPTOM CHECKLIST

On a scale of 1 to 5 , with 1 meaning "No symptoms" and 5 meaning "Severe Symptoms", how would you rate your current level of:

\begin{tabular}{|c|c|c|c|c|c|c|}
\hline & Pre & 0.15 & 0.3 & 0.45 & 0.6 & Post \\
\hline $\begin{array}{l}\text { General } \\
\text { discomfort }\end{array}$ & & & & & & \\
\hline Fatigue & & & & & & \\
\hline Headache & & & & & & \\
\hline Eyestrain & & & & & & \\
\hline Nausea & & & & & & \\
\hline Dizziness & & & & & & \\
\hline Difficulty fusing & & & & & & \\
\hline
\end{tabular}

Figure B.4: Page 1 of 2. The Questionnaire used for session 1 part 1 of the first user study 
148APPENDIX B. ADVERTISEMENTS, FORMS ANDQUESTIONNAIRES

\begin{tabular}{|c|c|c|c|c|c|c|}
\hline & $0.15->0.3$ & $0.15->0.45$ & $0.15->0.6$ & $0.3->0.45$ & $0.3->0.6$ & $0.45->0.6$ \\
\hline General discomf & & & & & & \\
\hline Fatigue & & & & & & \\
\hline Headache & & & & & & \\
\hline Eyestrain & & & & & & \\
\hline Nausea & & & & & & \\
\hline Dizziness & & & & & & \\
\hline Difficulty fusing & & & & & & \\
\hline
\end{tabular}

Other Comments:

Figure B.5: Page 2 of 2. The Questionnaire used for session 1 part 2 of the first user study 


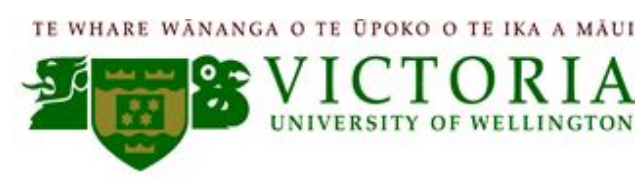

Investigating Discomfort in Head Mounted Displays

Ryan Sumner; Masters research project; Victoria University of Wellington

Participant Name:

Gender:

Age:

Questionnaire 2

SYMPTOM CHECKLIST

On a scale of 1 to 5 , with 1 meaning "No symptoms" and 5 meaning "Severe Symptoms", how would you rate your current level of:

\begin{tabular}{|c|c|c|c|c|c|c|}
\hline Distances & $0.15->0.3$ & $0.15->0.45$ & $0.15->0.6$ & $0.3->0.45$ & $0.3->0.6$ & $0.45->0.6$ \\
\hline Key & 1 & 2 & 3 & 4 & 5 & 6 \\
\hline
\end{tabular}

\begin{tabular}{|c|c|c|c|c|c|c|c|c|}
\hline & Pre & 1 & 2 & 3 & 4 & 5 & 6 & Post \\
\hline $\begin{array}{l}\text { General } \\
\text { discomfort }\end{array}$ & & & & & & & & \\
\hline Fatigue & & & & & & & & \\
\hline Headache & & & & & & & & \\
\hline Eyestrain & & & & & & & & \\
\hline Nausea & & & & & & & & \\
\hline Dizziness & & & & & & & & \\
\hline Difficulty fusing & & & & & & & & \\
\hline
\end{tabular}

Figure B.6: Page 1 of 2. The Questionnaire used for session 2 of the first user study 


\begin{tabular}{|l|l|l|l|l|l|l|l|l|}
\hline & Pre & $\mathbf{1}$ & $\mathbf{2}$ & $\mathbf{3}$ & $\mathbf{4}$ & $\mathbf{5}$ & $\mathbf{6}$ & Post \\
\hline General discomfort & & & & & & & & \\
\hline Fatigue & & & & & & & & \\
\hline Headache & & & & & & & & \\
\hline Eyestrain & & & & & & & & \\
\hline Nausea & & & & & & & & \\
\hline Dizziness & & & & & & & & \\
\hline Difficulty fusing & & & & & & & & \\
\hline
\end{tabular}

\begin{tabular}{|l|l|l|l|l|l|l|l|l|}
\hline & Pre & $\mathbf{1}$ & $\mathbf{2}$ & $\mathbf{3}$ & $\mathbf{4}$ & $\mathbf{5}$ & $\mathbf{6}$ & Post \\
\hline General discomfort & & & & & & & & \\
\hline Fatigue & & & & & & & & \\
\hline Headache & & & & & & & & \\
\hline Eyestrain & & & & & & & & \\
\hline Nausea & & & & & & & & \\
\hline Dizziness & & & & & & & & \\
\hline Difficulty fusing & & & & & & & & \\
\hline
\end{tabular}

Other Comments:

Preference:

Why?

Figure B.7: Page 2 of 2. The Questionnaire used for session 2 of the first user study 
Ryan Sumner; Masters research project; Victoria University of Wellington

Participant Name:

Gaming Experience:

Interpolation Speed:
Gender:

IPD:

Age:

Glasses:

IPD Offset:

"From modes 1, 2 or 3; rank your preference (if any) of the 'best' and 'worst' in terms of":

\begin{tabular}{|l|l|l|l|l|l|l|}
\hline Questions & $\mathbf{0 . 1 5 - 0 . 3 0}$ & $\mathbf{0 . 1 5 - 0 . 4 5}$ & $\mathbf{0 . 1 5 - 0 . 6 0}$ & $\mathbf{0 . 3 0 - 0 . 4 5}$ & $\mathbf{0 . 3 0 - 0 . 6 0}$ & $\mathbf{0 . 4 5 - 0 . 6 0}$ \\
\hline General Discomfort & & & & & & \\
\hline Eyestrain & & & & & & \\
\hline Nausea & & & & & & \\
\hline Dizziness & & & & & & \\
\hline Difficulty Fusing & & & & & & \\
\hline Fusion Time & & & & & & \\
\hline Overall & & & & & & \\
\hline
\end{tabular}

"From modes 1 or 2; rank your preference (if any) of the 'best' and 'worst' in terms of":

\begin{tabular}{|l|l|l|l|}
\hline Questions & Slow & Medium & Fast \\
\hline General Discomfort & & & \\
\hline Eyestrain & & & \\
\hline Nausea & & & \\
\hline Dizziness & & & \\
\hline Difficulty Fusing & & & \\
\hline Fusion Time & & & \\
\hline Overall & & & \\
\hline
\end{tabular}

Figure B.8: The Questionnaire used for the second user study 